\title{
Optical absorption and luminescence in diamond
}

\author{
JOHN WALKER $\uparrow$
}

Groupe de Physique des Solides de l'Ecole Normale Supérieureł, Université Paris VII, 2 place Jussieu, 75221 Paris Cedex 05, France

\begin{abstract}
The optical properties of diamond are important in theoretical solid-state physics, geology, electronics and diamond synthesis.

This review outlines some relevant aspects of theoretical physics-molecular orbital and other calculations of the properties of point defects, symmetry and group theory, zero-phonon lines and the configuration coordinate diagram.

The experimentally observed spectra can conveniently be divided into infrared $(<1 \mathrm{eV})$, which are observed in absorption and are mainly vibrational, and 'visible' $(1-5.5 \mathrm{eV})$, which are vibronic and are observed in both absorption and luminescence. 'Visible' spectra include those present naturally, those induced by irradiation, and those induced by irradiation plus annealing.

This review covers all the important point defects observed optically in diamond, and includes some results which are not generally available. The relevance of the experimental results to the current controversy about how to calculate the properties of point defects is brought out.
\end{abstract}

This review was received in April 1978.

† Present address: 24 Betchworth Ave., Earley, Reading RG6 2RJ, UK.

$\ddagger$ Laboratory associated with the CNRS. 


\section{Contents}

$\begin{array}{ll}\text { 1. Introduction } & \text { Page } \\ 1607\end{array}$

1.1. Why study optical absorption and luminescence in diamond? $\quad . \quad$. 1607

1.2. Previous work . . . . . . . . . 1607

1.3. The classification of diamond . . . . . . . . . . 1607

1.4. Impurities in diamond . . . . . . . . . . . 1609

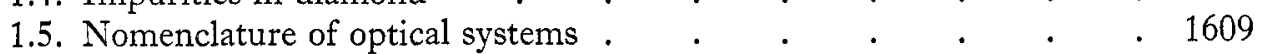

1.6. Paramagnetic resonance . . . . . . . . . 1610

1.7. Synthetic diamond . . . . . . . . . . . 1610

1.8. Ion implantation and irradiation . . . . . . . 1610

2. Theory . . . . . . . . . . . . 1611

2.1. The configuration coordinate diagram, zero-phonon lines and the Huang-Rhys factor . $\quad . \quad$. $\quad . \quad$. . . . . 1611

2.2. Symmetry and group theory . . . . . . . . 1613

2.3. The Jahn-Teller effect . . . . . . . . 1613

2.4. Calculations of the properties of defects. $\quad . \quad$. $\quad . \quad$. $\quad$. 1615

2.5. Symmetry lowering perturbations . . . . . . . 1616

3. Infrared spectra (mainly vibrational) . . . . . . . . . . 1616

3.1. The lattice bands . . . . . . . . . . . . . 1617

3.2. Defect-induced absorption . . . . . . . . . . . 1617

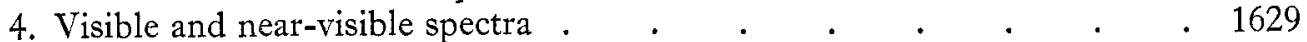

4.1. Unirradiated diamond . . . . . . . . . . . . 1629

4.2. Irradiated diamond . . . . . . . . . . . . . . 1636

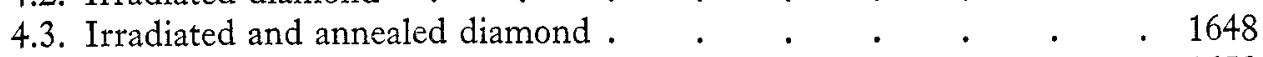

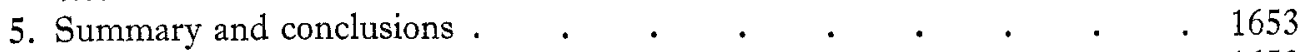

Acknowledgments . $\quad . \quad$. $\quad . \quad$. . . . . 1653

References . $\quad . \quad$. $\quad . \quad . \quad . \quad . \quad . \quad . \quad 1654$ 


\section{Introduction}

\subsection{Why study optical absorption and luminescence in diamond?}

Diamonds ought to be transparent over most of the spectrum, but many are not. They may absorb and luminesce naturally, or after particle bombardment, and we want to know why. Secondly, diamond is a testing ground for theoretical calculations on point defects, which are responsible for this absorption and luminescence. Thirdly, the mystery of the vanishing interstitial in silicon (Watkins 1965) may be solved by reference to diamond. Fourthly, the study of the different spectra manifested by natural and synthetic diamonds may tell us more about how the former were created, and how to grow the latter more efficiently.

So there are good reasons for studying diamond; but there are difficulties too. Synthetic diamonds are available $(\$ 1.7)$ but nitrogen and boron are the only impurities which can be introduced into them with any degree of control.

\subsection{Previous work}

There are a number of reviews on the physical properties of diamond, including Berman (1965), Burls (1967) and Field (1979). Reviews particularly relevant to this article are those by Clark (1965), Clark and Mitchell (1971a, b, 1976) and Clark et al (1978) on radiation damage, by Davies (1972a, b) on nitrogen in diamond, and by Kemmey and Wedepohl (1965), Vavilov (1975) and Vavilov and Konorova (1976) on semiconducting diamond. Davies (1977a) covers similar ground to the present review.

\subsection{The classification of diamond}

No two diamonds are identical, but many are sufficiently similar for a qualitative classification system to be useful, although some crystals do not fit comfortably into any one category. The system was proposed by Robertson et al (1934) and elaborated by various workers. It is outlined in table 1 . Although the system was based on measurable macroscopic properties of diamonds, it has become clear that nitrogen and boron impurities are the microscopic bases of the classification.

This is the generally accepted classification scheme. It is worth emphasising that types $\mathrm{Ib}$ and IIb are definitive categories, with a particular qualification for membership. Types Ia and IIa are residual classes (although they contain the vast majority of natural diamonds). Type Ia in particular is a mixed bag. As table 1 shows, such diamonds contain aggregates on (100) planes (the 'platelets') which are directly observable in the electron microscope (Evans and Phaal 1962a, b) and which give rise to the anomalous x-ray diffraction 'spikes' (Hoerni and Wooster 1955). They also exhibit defect-induced optical absorption of at least five types: the A, B1 and B2 bands in the infrared, and the $\mathrm{N} 3$ system and the secondary absorption edge in the uv (Davies 1972a, b). These are all discussed more fully in $\S 3$, where it is shown that not all these properties correlate. Consequently, extensions to the classification scheme have been suggested by Sobolev et al (1969a), Davies (1972a, b) and Klyuev et al (1972). None of these modifications are generally accepted and they are not consistent with each other. 
Table 1. The classification of diamonds. Crystals of mixed types may occur (see figure 1).

\begin{tabular}{|c|c|c|c|c|c|}
\hline \multirow[b]{2}{*}{ Type } & \multirow[b]{2}{*}{ Defining feature } & \multicolumn{2}{|c|}{ Optical absorption } & \multirow[b]{2}{*}{ ESR } & \multirow{2}{*}{$\begin{array}{l}\text { Typical } \\
\text { electrical } \\
\text { resistance }\end{array}$} \\
\hline & & Visible & Infrared & & \\
\hline Ia & $\begin{array}{l}\text { Aggregated } \\
\text { nitrogen, } \\
\text { x-ray diffraction } \\
\text { spikes, 'platelets' }\end{array}$ & $\begin{array}{l}\text { Secondary } \\
\text { absorption edge } \\
\text { at about } 300 \mathrm{~nm} \text {, } \\
\text { N3 }(415 \mathrm{~nm})\end{array}$ & $\begin{array}{l}\text { A bands } \\
\text { B1 and } \\
\text { B2 peaks }\end{array}$ & 一 & $>10^{15} \Omega \mathrm{cm}$ \\
\hline $\mathrm{Ib}$ & $\begin{array}{l}\text { Paramagnetic } \\
\text { singly } \\
\text { substitutional } \\
\text { nitrogen }\end{array}$ & $\begin{array}{l}\text { Strong } \\
\text { featureless } \\
\text { absorption } \\
\text { increasing in } \\
\text { intensity at } \\
\text { higher photon } \\
\text { energy }\end{array}$ & $\begin{array}{l}\text { Peaks at } \\
0 \cdot 14 \\
\text { and } \\
0 \cdot 167 \mathrm{eV}\end{array}$ & $\begin{array}{l}\text { Paramagnetic } \\
\text { nitrogen }\end{array}$ & $>10^{15} \Omega \mathrm{cm}$ \\
\hline IIa & 一 & $\begin{array}{l}\text { Fundamental } \\
\text { absorption edge, } \\
5.5 \mathrm{eV} \text {, often } \\
\text { with weak } \\
\text { featureless 'tail' }\end{array}$ & $\begin{array}{l}\text { Lattice } \\
\text { bands } \\
\text { only }\end{array}$ & $\begin{array}{l}\text { Single } \\
\text { isotropic } \\
\text { line }\end{array}$ & $>10^{13} \Omega \mathrm{cm}$ \\
\hline IIb & $\begin{array}{l}\text { p-type } \\
\text { semiconductor }\end{array}$ & $\begin{array}{l}\text { Fundamental } \\
\text { absorption edge, } \\
5 \cdot 5 \mathrm{eV} \text {, absorption } \\
\text { at red end of } \\
\text { spectrum }\end{array}$ & $\begin{array}{l}\text { Acceptor } \\
\text { bands, } \\
\text { free } \\
\text { carrier } \\
\text { absorption }\end{array}$ & None & $150 \Omega \mathrm{cm}$ \\
\hline
\end{tabular}

The best compromise therefore seems to be to adopt the subclassifications IaA, IaB1, IaB2 and IaB3, the characteristics of which are detailed in table 2. Of course, for many purposes the simple Ia category will suffice.

Although they are not given a special category, 'smoky-brown' diamonds appar-

Table 2. Proposed subclassification of type Ia diamonds and the impurity-induced A, B1 and $\mathrm{B} 2$ infrared absorption bands. Different authors quote slightly different values of wavenumber. It is not known whether the $780 \mathrm{~cm}^{-1}$ feature is $\mathrm{B} 1$ or $\mathrm{B} 2$; according to Charette (1961a) it is neither. Sobolev et al (1969a) refer to N3-containing diamonds as type IaB3, but $\mathrm{G}$ Davies (personal communication) has pointed out that there is no evidence for a subclass of diamonds containing predominantly $\mathrm{N} 3$ centres.

\begin{tabular}{|c|c|c|c|}
\hline \multirow[b]{2}{*}{ Type } & \multicolumn{2}{|c|}{ Optical absorption } & \multirow[b]{2}{*}{ Other } \\
\hline & $\mathrm{IR}$ & $\mathrm{uV}$ & \\
\hline $\mathrm{IaA}$ & $\begin{array}{l}480,1100,1215 \\
1282 \mathrm{~cm}^{-1}\end{array}$ & $\begin{array}{l}\text { N5, N6, N7, N8 and } \\
\text { secondary absorption } \\
\text { edge at } \lambda<330 \mathrm{~nm}\end{array}$ & \\
\hline $\mathrm{IaB} 1$ & $\begin{array}{l}1010,1100,1175 \\
1332 \mathrm{~cm}^{-1}\end{array}$ & (N9 ?) & \\
\hline $\mathrm{IaB} 2$ & $\begin{array}{l}330,1365 \\
1430 \mathrm{~cm}^{-1} \\
1.25 \mathrm{eV}\end{array}$ & $\begin{array}{l}\text { Bands at } \\
260-280 \mathrm{~nm}\end{array}$ & $\begin{array}{l}\text { x-ray } \\
\text { spikes, } \\
\text { platelets }\end{array}$ \\
\hline IaB3 & 一 & $\mathrm{N} 2, \mathrm{~N} 3, \mathrm{~N} 4$ & \\
\hline
\end{tabular}


ently form a distinct subclass, with specific EPR, optical and other properties (Shcherbakova et al 1975).

Finally, we note that many diamonds are inhomogeneous. That is, parts of the diamond may be type II, other parts type I (Berman 1965, Takagi and Lang 1964, Evans 1976, Hanley et al 1977); some parts may be 'platelet-rich' (type IaB2), others 'platelet-free' (type IaA or IaB1) and so on (figure 1 (plate)). This inhomogeneity has bedevilled many attempts to correlate particular features of different diamonds; experimenters must be wary of it.

\subsection{Impurities in diamond}

Many impurities have been detected in diamond, though one should emphasise that gem-quality crystals are surprisingly pure considering their origin. Apart from nitrogen and boron no element has been found in a concentration greater than $100 \mathrm{ppm}$ (the carbon atom is so small that few atoms can fit onto a normal lattice site without a great deal of strain, and solubilities are therefore low). The fact that so many elements have been detected is a tribute to the sensitivity of the methods used.

The early work used spectrographic analysis or mass spectrometry and residual gas analysis. More recently, the powerful technique of activation analysis has extended earlier results. Fesq et al (1973) have conveniently tabulated the elements observed by themselves and by previous workers, so it will be sufficient here to discuss only the impurities of particular interest. Nitrogen is dealt with in $\S \S 1.3,3$ and 4. Hydrogen is discussed by Sellschop et al (1977).

Boron is the element responsible for p-type semiconductivity in diamond (Chrenko 1973, Lightowlers and Collins 1976). Aluminium was originally thought to be the acceptor but was almost certainly present as metallic inclusions in synthetic diamond (Chrenko 1973) and as submicroscopic aluminosilicate droplets in natural crystals (Sellschop 1975, Fesq et al 1975) rather than as dispersed single atoms. We cannot say that aluminium cannot be an acceptor in diamond (although recent calculations by Astier and Bourgoin (1976) make it unlikely), but boron plays the dominant role.

Oxygen in diamond has been detected by vacuum fusion and by activation analysis (Kaiser and Bond 1959, Sellschop et al 1974, Sellschop 1975). Some of the oxygen is present as a gas in visible inclusions (Melton and Giardini 1974). Some is in chemical combination as submicroscopic aluminosilicate inclusions, though there is a persistent excess over and above what can be contained in the aluminosilicate (Sellschop 1975). This excess presumably could be present substitutionally or interstitially in the diamond lattice (oxygen is a well-known impurity in silicon).

More recently, in a study of 'inclusion-free' diamonds, Melton and Giardini (1976) have claimed that oxygen, and not nitrogen, is the dominant impurity in diamond. This seems unlikely given the internal consistency of Kaiser and Bond's results, and the corroboration by Lightowlers and Dean using a different technique, but further work is certainly desirable to resolve the discrepancy (Walker 1976b).

\subsection{Nomenclature of optical systems}

It is usual to label only the zero-phonon line (\$2.1) of an optical system. Clark et al $(1956 \mathrm{a}, \mathrm{b})$ introduced letters and numbers to label all the optical absorption lines they observed (table 3 ). However, many other zero-phonon lines have since been observed, particularly in luminescence, and the nomenclature adopted, though often descriptive, 


\section{f Walker}

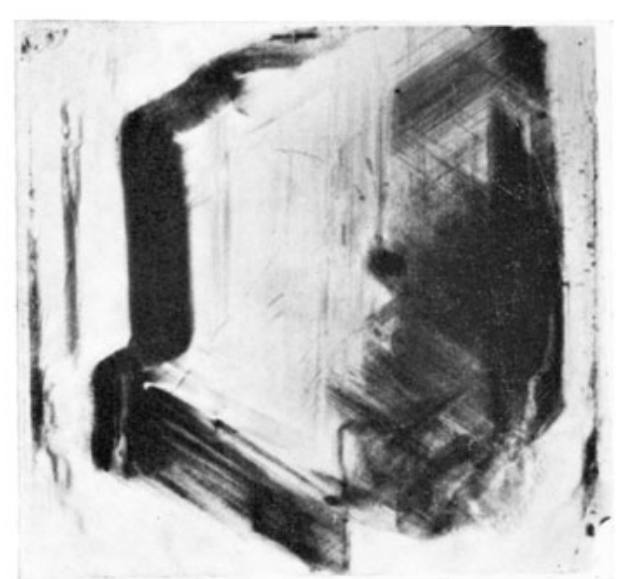

(a)

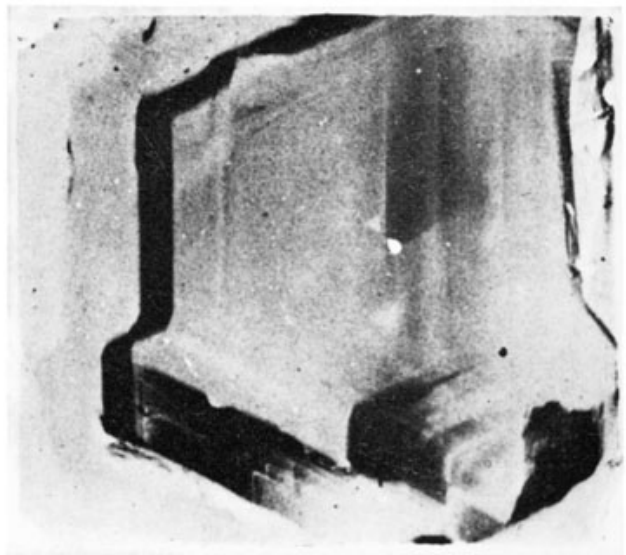

(c)

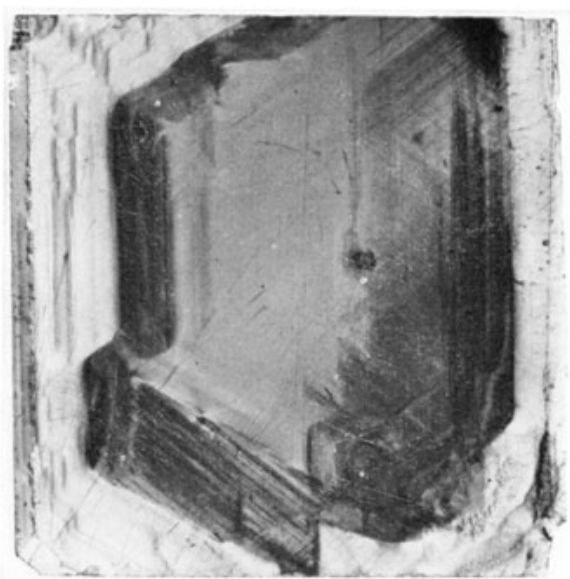

(b)

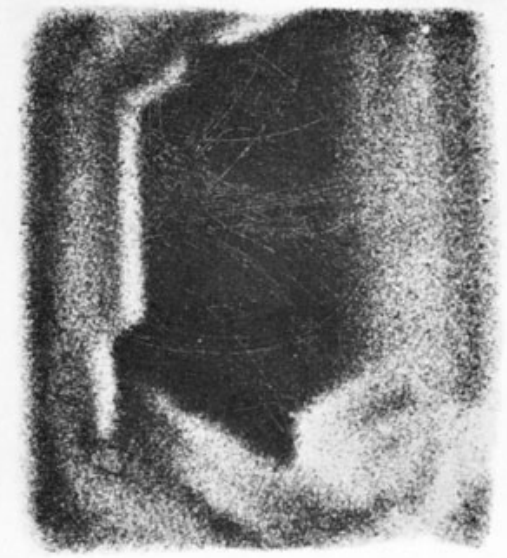

(d)

Figure 1. An inhomogeneous diamond, as studied by various techniques. (a) Bragg topograph, $\mathrm{MoK} \alpha$ radiation, (b) Bragg topograph, $\mathrm{CuK} \alpha$ radiation, (c) ultraviolet transmission topograph, $(d)$ 'spike' topograph. These topographs are discussed in detail by Takagi and Lang (1964), from whose paper they are taken (see also Hanley et al 1977). 
Table 3. The nomenclature for zero-phonon lines introduced by Clark et al (1965a, b). R10 is more frequently called ND1.

\begin{tabular}{|c|c|c|}
\hline Letter & Significance & Range \\
\hline $\mathbf{N}$ & Natural diamond & $\mathrm{N} 1(1.50 \mathrm{eV})$ to $\mathrm{N} 9(5.26 \mathrm{eV})$ \\
\hline GR & $\begin{array}{l}\text { General radiation (induced in } \\
\text { all diamonds by irradiation) }\end{array}$ & GR1 $(1.67 \mathrm{eV})$ to $\mathrm{GR} 8(3.00 \mathrm{eV})$ \\
\hline $\mathbf{R}$ & $\begin{array}{l}\text { Radiation (masked in type Ia } \\
\text { diamond by the secondary } \\
\text { absorption edge) }\end{array}$ & $\mathrm{R} 9(3.04 \mathrm{eV})$ to $\mathrm{R} 11(3.99 \mathrm{eV})$ \\
\hline TR & $\begin{array}{l}\text { Type II radiation (not } \\
\text { observed in type I) }\end{array}$ & TR12 $(2.64 \mathrm{eV})$ to TR17 $(2.83 \mathrm{eV})$ \\
\hline $\mathrm{H}$ & $\begin{array}{l}\text { Heat treatment (preceded by } \\
\text { irradiation) }\end{array}$ & $\mathrm{H} 1(0.18 \mathrm{eV})$ to $\mathrm{H} 18(3.56 \mathrm{eV})$ \\
\hline
\end{tabular}

is not consistent with Clark et al. Some are labelled by letters, others by their wavelength or photon energy.

\subsection{Paramagnetic resonance}

Electron paramagnetic resonance (EPR) or electron spin resonance (ESR)-the terms are used interchangeably because it is usually spin paramagnetism one is concerned with in solids-is a powerful technique for the identification and study of point defects in solids. It has been used with great success in silicon (Watkins 1965) and diamond (Loubser and van Wyk 1978).

\subsection{Synthetic diamond}

The successful synthesis of diamond was reported by Bundy et al (1955). Progress since that time has been reviewed by Bundy et al (1973). The production process is now commonplace and for some industrial applications the synthetic material is better than the natural. Synthetics are usually of type Ib, which is surprising because Ib natural crystals are very rare. Semiconducting crystals can be made by doping with boron (the natural variety again being rare), confirming that boron forms the acceptor centre in natural semiconducting diamonds. Weakly semiconducting crystals can be made by doping with aluminium; it is usually assumed that this metal is not electrically active but 'getters' any nitrogen present, thus allowing any boron to remain uncompensated.

Because of the relatively poor quality and small size of synthetic diamonds they are not generally convenient for optical or ESR experiments though a certain amount of work has been reported (du Preez 1965, Bourgoin et al 1972, Wight et al 1971, Bourgoin and Massarani 1976, Massarani and Bourgoin 1976), and, apart from nitrogen and boron, it is not possible at present to introduce impurities in a controlled manner, which is a considerable handicap. This is one of the reasons why irradiation is so important, as we will see.

\subsection{Ion implantation and irradiation}

Because of the difficulties of doping diamonds with impurities by diffusion or during growth, some workers have tried ion implantation. In silicon the technique 
has been used successfully but in diamond progress has been slower, the major problem being that annealing after implantation to remove radiation damage may result in conversion to graphite; the successes claimed in early work were exaggerated. The induced conductivity was almost certainly due to radiation damage or graphite rather than genuine impurity doping (Clark and Mitchell 1971a, b, Bourgoin and Walker 1976). However, Vavilov et al (1974) apparently succeeded in implanting boron, and lithium appears promising (Vavilov et al 1972). But there are still difficulties. Potential dopants have atomic radii larger than carbon, so solubilities will be low.

Irradiation is the only technique which has met with significant success in modifying the optical properties of diamond. Electrons are most convenient because their direction, energy and flux can be easily controlled, and because they produce individual point defects, resulting in sharp lines in the crystal. Neutrons give broad lines, presumably because many defects are produced close together, leading to distorted surroundings and strains. Annealing after irradiation allows defects to become mobile and to migrate through the crystal, becoming trapped at impurities to form new complexes and hence new spectra. This implies that the initial irradiation should be at as low a temperature as possible, to inhibit migration during irradiation, which might lead to confusion about which are the primary defects (i.e. vacancies and interstitials). Most studies have used room-temperature irradiations, but one defect at least is mobile below that (Lomer and Wild 1971, Massarani and Bourgoin 1976, Lomer and Welbourn 1976).

We will see in $\$ 4.3$ that some spectra have been ascribed to the vacancy trapped at specific nitrogen complexes, but only one radiation-induced defect has been linked to boron-the $2 \mathrm{BD}$ centre $(\$ 4.2)$. This is presumably because natural type $\mathrm{IIb}$ diamonds are rare, and furthermore contain only a low concentration (typically $10 \mathrm{ppm}$ ) of boron acceptors. Although synthetic diamonds are not generally suitable for optical experiments $(\$ 1.7)$, it seems worthwhile to study irradiated synthetic type IIb to see if they display characteristic spectra.

\section{Theory}

\subsection{The configuration coordinate diagram, zero-phonon lines and the Huang-Rhys factor}

The configuration coordinate diagram (figure 2(a)) is useful in explaining optical spectra of defects in solids. A simplified treatment is given here. For further details see Sturge (1967), Curie (1975) and Stoneham (1975).

At $T=0 \mathrm{~K}$ only the lowest 'vibronic' (vibrational-electronic) state $(m=0)$ is populated. The normalised transition probability to the $n$th level of the excited state is:

$$
W_{n 0}=S^{n} \mathrm{e}^{-S} / n !
$$

which leads to an absorption spectrum consisting of peaks at $E=E_{0}+n \hbar w$ (figure $2(b)$ ). In diamond these peaks are usually resolved, even at room temperature; in some cases (e.g. F centres in alkali halides), the individual transitions are broadened and only their envelope is seen. The most probable (Franck-Condon) transition is the vertical one, $\mathrm{A} \rightarrow \mathrm{B}$ in figure $2(a)$, with $n=S$. The defect can then relax to $\mathrm{C}$ by emission of $S$ phonons, decay to D by emission of a photon, and relax back to $\mathrm{A}$ by emission of another $S$ phonons. $S$-the Huang-Rhys factor-is a measure of the strength of the electron-phonon coupling. 
A very important transition occurs between the levels $m=0$ and $n=0$. This is the zero-phonon line $(Z$ in figure $2(b)$ )-a purely electronic transition. Since no phonons are involved, it is very sharp. In principle, its width is determined by the lifetime of the excited state, though in practice random strains in the crystal broaden it to values of about $1 \mathrm{meV}$. Nonetheless it is considerably sharper than the phononassisted transitions, and the way it splits under various perturbations gives valuable information about the symmetry of the defect (\$2.5). At high temperatures or large $S$ (strong electron-phonon coupling) the zero-phonon line is weak compared to the
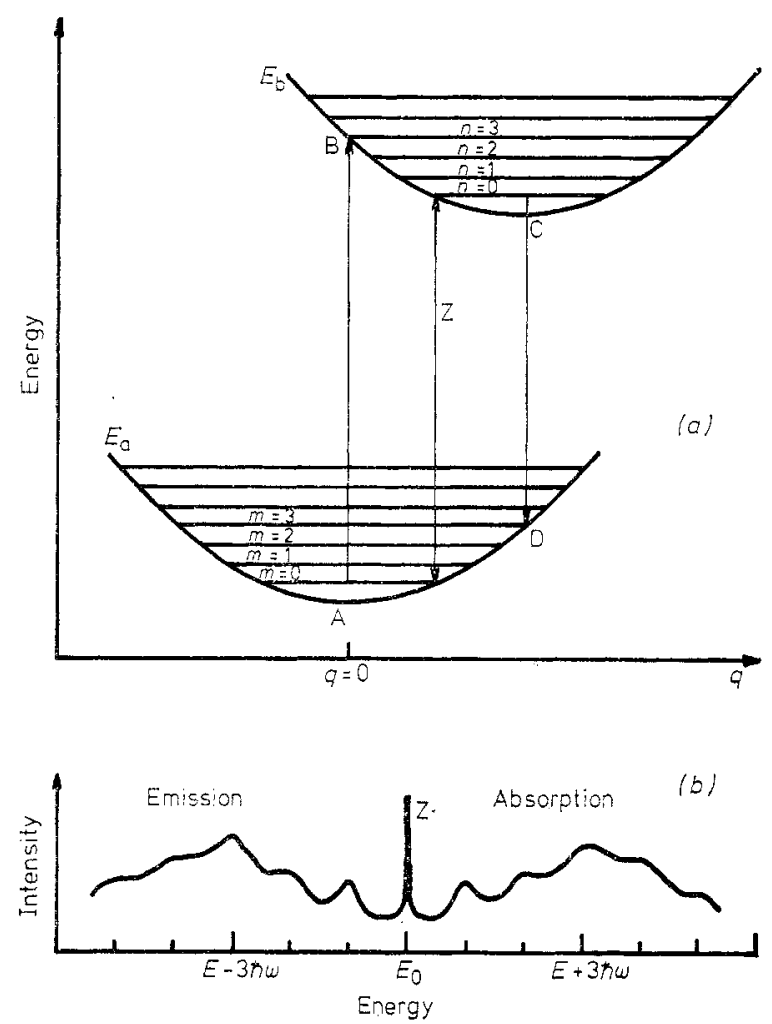

Figure 2. (a) A configuration coordinate diagram showing the ground $\left(E_{\mathrm{a}}\right)$ and excited $\left(E_{\mathrm{b}}\right)$ electronic states with their vibrational sublevels. $Z$ is the zero-phonon line. $(b)$ The corresponding absorption and emission bands. Note that their maxima correspond to the transitions $A \rightarrow B$ and $C \rightarrow D$. See text.

phonon sidebands. For $S>6$ it is usually thought to be unobservable, though $G$ Davies (personal communication) has pointed out that the UV zero-phonon lines of the IaA nitrogen defect (\$3.2.1.1) are plainly visible though $S$ is greater than 10 .

In absorption the phonon-assisted transitions occur at higher energy than the zero-phonon line, whereas in emission they are at lower energy (the Stokes shift) (figure $2(b)$ ). On the simple model, there should be mirror symmetry about the zero-phonon line. If there is not, non-linear coupling or a Jahn-Teller effect must be present. This is the case for some defects in diamond ( $\$ 4)$.

The Huang-Rhys factor is an important characteristic of an optical system. It indicates the fractional intensity of the zero-phonon line, the magnitude of the Stokes 
shift and the width of the phonon sideband (all measured at $T=0 \mathrm{~K}$ ). Thus:

$$
\begin{aligned}
I_{\mathrm{zpl} 1} & =I_{\text {tot }} \exp (-S) \\
E_{\text {abs }}-E_{\text {emiss }} & =2 S \hbar \omega \\
\Delta^{2} & =5.6(\hbar \omega)^{2} S .
\end{aligned}
$$

In some cases (for example, the GR1 system-see $\$ 4.2$ ), the values of $S$ calculated by these three methods are not in agreement, indicating that the simple model does not apply-there is therefore electronic degeneracy, a Jahn-Teller effect $(\$ 2.3)$ or a change in force constants.

\subsection{Symmetry and group theory}

It is impossible to deal here with group theory and crystal symmetry, but some brief explanations of the notation to be used will be given. For further details, see Tinkham (1964) and Heine (1964).

The diamond lattice is face-centred-cubic, with two atoms per unit cell, at $0,0,0$ and $a / 4, a / 4, a / 4$. (Alternatively, we may say that the structure contains two interpenetrating face-centred-cubic lattices with origins at $0,0,0$ and $a / 4, a / 4, a / 4$.) The space group is $\mathrm{O}_{\mathrm{h}}{ }^{7}$. The point group of the lattice is $\mathrm{O}_{\mathrm{h}}$; the point group of an individual lattice atom site is $T_{d}$. The distinction is important because some point defects may have symmetries which are not subgroups of $T_{d}$, though they must be subgroups of $\mathrm{O}_{\mathrm{h}}$ (Henry and Lonsdale $1965 \mathrm{p} 340$ ). For example, the A centre, which is probably a nitrogen pair, has $\mathrm{D}_{3 \mathrm{~d}}$ symmetry ( $\left.\S 3.2\right)$, as does the so-called 'hexagonal' interstitial site (Weigel et al 1973).

The defining symmetry elements of $T_{d}$ and its subgroups are given in table 4 . The non-cubic subgroups of $\mathrm{O}_{\mathrm{h}}$ are $\mathrm{D}_{4 \mathrm{~h}}, \mathrm{D}_{4}, \mathrm{C}_{4 \mathrm{v}}, \mathrm{D}_{2 \mathrm{~d}}, \mathrm{C}_{4 \mathrm{~h}}, \mathrm{C}_{4}, \mathrm{~S}_{4}$ (tetragonal); $\mathrm{D}_{3 \mathrm{~d}}, \mathrm{D}_{3}, \mathrm{C}_{3 \mathrm{v}}, \mathrm{C}_{3 \mathrm{i}}, \mathrm{C}_{3}$ (trigonal); $\mathrm{D}_{2 \mathrm{~h}}, \mathrm{D}_{2}, \mathrm{C}_{2 \mathrm{v}}$ (rhombic); $\mathrm{C}_{2 \mathrm{~h}}, \mathrm{C}_{2}, \mathrm{C}_{\mathrm{s}}$ (monoclinic); $\mathrm{C}_{\mathrm{i}}, \mathrm{C}_{1}$ (triclinic).

In a solid the normal spectroscopic labels of atomic energy levels cannot be used because the crystal electrostatic field quenches orbital angular momentum. The maximum orbital degeneracy is three in $T_{d}$, two in $D_{2 d}$ and $C_{3 v}$, and one in the other subgroups of $T_{d}$. The spectroscopic labels used in solids are group-theoretical onesthe characters of the irreducible representations of the point groups. There are various systems of notation. In the Mulliken system $T$ denotes triply degenerate orbital levels, E doubly degenerate levels, and A and B non-degenerate levels. Subscripts can be added to distinguish different labels with the same degeneracy, and (where appropriate) to denote odd or even parity. In $T_{d}$ there are five labels: $A_{1}, A_{2}$, $E, T_{1}$ and $T_{2}$. The allowed transitions are $A_{1}-T_{2}, A_{2}-T_{1}, E-T_{1}, E-T_{2}, T_{1}-T_{1}, T_{2}-T_{2}$ and $\mathrm{T}_{1}-\mathrm{T}_{2}$. For lower symmetries the labels and allowed transitions are given by Tinkham (1964), Heine (1964) and Kaplyanskii (1964a,b). Superscripts $(2 S+1)$ are used in the conventional way to denote spin degeneracy, e.g. ${ }^{3} \mathrm{~T}_{1}$ or ${ }^{1} \mathrm{E}$. Another system uses $\Gamma_{1}, \Gamma_{2}, \ldots \Gamma_{5}$ instead of $A, E$ and $T$, plus extra labels $\Gamma_{6}, \Gamma_{7}, \Gamma_{8}$ for the double group ${ }^{2} \mathrm{~T}_{\mathrm{d}}$.

\subsection{The fahn-Teller effect}

Several defects in diamond manifest a Jahn-Teller effect. The theorem states that any symmetric molecule or point defect with degenerate electronic energy levels 
Table 4. The table uses the Schoenflies notation (Tinkham 1964), $C_{n}$ and $S_{n}$ denote proper and improper $n$-fold notations about a specific axis; $\sigma_{\mathrm{h}}$ and $\sigma_{\mathrm{v}}$ are reflections in the 'horizontal' and 'vertical' axes ('vertical' is the axis of highest symmetry); $\sigma_{\mathrm{d}}$ is a $\sigma_{\mathrm{v}}$ which bisects two two-fold axes. The point groups are labelled according to the symmetry elements they contain, $T$ denoting tetrahedron (more than one axis with $n>2$ ), $\mathrm{D}$ dihedral (more than 1 axis with $n=2$ ). $\pi, \sigma$ and $\mathrm{V}$ denote linear (parallel to the axis of the centre), planar (perpendicular to the axis of the centre), and volume oscillators. See Kaplyanskii (1964a, b) for further details.

\begin{tabular}{|c|c|c|c|}
\hline $\begin{array}{l}\text { Symmetry } \\
\text { system }\end{array}$ & $\begin{array}{l}\text { Point } \\
\text { groups }\end{array}$ & $\begin{array}{l}\text { Defining } \\
\text { symmetry } \\
\text { elements }\end{array}$ & $\begin{array}{l}\text { Electric } \\
\text { dipole } \\
\text { oscillators }\end{array}$ \\
\hline Tetrahedral & $T_{d}$ & $\begin{array}{l}\mathrm{C}_{4}\langle 100\rangle \\
\mathrm{C}_{3}\langle 111\rangle\end{array}$ & $\begin{array}{l}\mathrm{V} \\
\text { (isotropic) }\end{array}$ \\
\hline Tetragonal & $\begin{array}{l}\mathrm{D}_{2 \mathrm{~d}} \\
\mathrm{~S}_{4}\end{array}$ & $\begin{array}{l}\mathrm{C}_{4}\langle 100\rangle \\
\mathrm{S}_{4}\langle 100\rangle\end{array}$ & $\left\{\begin{array}{l}\pi\langle 100\rangle \\
\sigma\langle 100\rangle\end{array}\right.$ \\
\hline Trigonal & $\begin{array}{l}\mathrm{C}_{3 \mathrm{v}} \\
\mathrm{C}_{3}\end{array}$ & $\mathrm{C}_{3}\langle 111\rangle$ & $\left\{\begin{array}{l}\pi\langle 111\rangle \\
\sigma\langle 111\rangle \\
\mathrm{V}\end{array}\right.$ \\
\hline Rhombic I & $\begin{array}{l}\mathrm{C}_{2 \mathrm{v}} \\
\mathrm{D}_{2}\end{array}$ & $\begin{array}{l}\mathrm{C}_{2}\langle 100\rangle \\
\mathrm{C}_{2} \text { or } \sigma_{\mathrm{v}}\langle 011\rangle \\
\mathrm{C}_{2} \text { or } \sigma_{\mathrm{V}}\langle 0 \overline{1} 1\rangle\end{array}$ & $\begin{array}{l}\pi\langle 100\rangle \\
\pi\langle 011\rangle \\
\pi\langle 011\rangle\end{array}$ \\
\hline Rhombic II & $\begin{array}{l}\mathrm{C}_{2 \mathrm{v}} \\
\mathrm{D}_{2}\end{array}$ & $\begin{array}{l}\mathrm{C}_{2}\langle 100\rangle \\
\mathrm{C}_{2} \text { or } \sigma_{\mathrm{v}}\langle 010\rangle \\
\mathrm{C}_{2} \text { or } \sigma_{\mathrm{v}}\langle 001\rangle\end{array}$ & $\begin{array}{l}\pi\langle 100\rangle \\
\pi\langle 010\rangle \\
\pi\langle 001\rangle\end{array}$ \\
\hline Monoclinic I & $\begin{array}{l}\mathrm{C}_{2} \\
\sigma_{\mathrm{h}}\end{array}$ & $\mathrm{C}_{2}$ or $\sigma\langle 110\rangle$ & $\begin{array}{l}\pi\langle 110\rangle \\
\sigma \|\langle 110\rangle\end{array}$ \\
\hline Monoclinic II & $\begin{array}{l}\mathrm{C}_{2} \\
\sigma_{\mathrm{h}}\end{array}$ & $\mathrm{C}_{2}$ or $\sigma\langle 100\rangle$ & $\begin{array}{l}\pi\langle 001\rangle \\
\sigma \mid\langle 001\rangle\end{array}$ \\
\hline Triclinic & $\mathrm{C}_{1}$ & $\mathrm{E}$ & $\mathrm{V}$ \\
\hline
\end{tabular}

will distort in order to lift the degeneracy and lower its energy. The only exceptions are linear molecules and Kramers degeneracy. The operation of the theorem is indicated schematically in figure 3 , which shows a doubly degenerate state of energy $E_{0}$ which splits as a result of a distortion $Q$. The splitting for a small distortion is linear in $Q$, so one level has a lower energy $E_{-}$than the undistorted configuration. Elastic

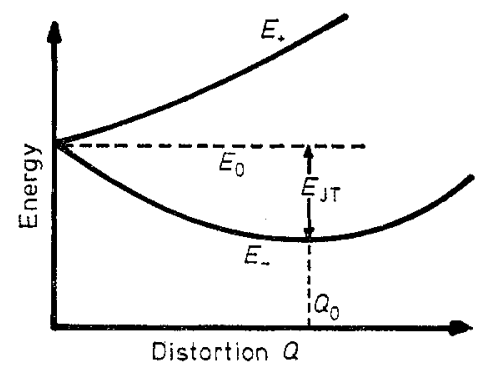

(a)

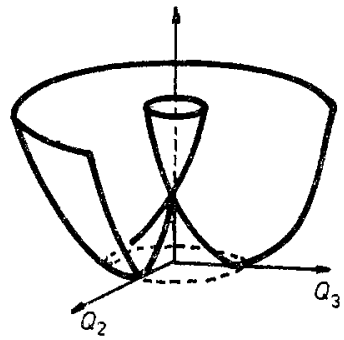

(b)

Figure 3. (a) Schematic representation of the lowering in energy $E_{\mathrm{J}}$ resulting from the splitting of a degenerate electronic level due to a Jahn-Teller distortion. (b) Revolution of (a) about the vertical axis-the 'Mexican Hat'. 
restoring forces which are quadratic in $Q$ limit the distortion to a finite value $Q_{0}$, and the consequent energy reduction is the Jahn-Teller energy $E_{\text {JT }}$. See Sturge (1967), Ham (1972), Englman (1971) and Stoneham (1975) for recent review articles.

There are four main ways in which the Jahn-Teller effect may manifest itself in point defects. Firstly, the symmetry observed may be lower than expected. This is the static Jahn-Teller effect. The substitutional nitrogen donor in diamond is an example (\$3.2). It might be expected to be tetrahedral, but the extra electron occupies an antibonding orbital localised on the nitrogen and one single carbon atom, resulting in a distortion to trigonal symmetry. There are four equivalent distortions, corresponding to the four $\langle 111\rangle$ directions. Secondly, if the temperature is high enough or the energy barrier low enough, reorientation between equivalent distortions may occur. This is the dynamic Jahn-Teller effect. The original symmetry has effectively been restored, the electronic degeneracy being replaced by an equivalent vibronic degeneracy. It happens to the nitrogen donor in diamond at about $800 \mathrm{~K}$ (Shul'man et al 1967, Loubser and Van Ryneveld 1967), and is thought to be important at the GR1 centre (\$4.2).

The third manifestation of the Jahn-Teller effect was not recognised as such until much later, from the work of Ham (1972), who showed that a dynamic Jahn-Teller effect can cause a 'quenching' of orbital angular momentum, spin-orbit splitting and uniaxial stress splitting (the Ham effect). Fourthly, it may affect vibronic band shapes $(\$ 2.1)$.

\subsection{Calculations of the properties of defects}

The spacing and symmetry of energy levels in atoms and molecules can now be calculated with considerable accuracy. But the analogous problem in semiconductors - the energy levels of point defects-has been solved only in certain special cases. 'Shallow' defects (i.e. with energy levels within $0.1 \mathrm{eV}$ of band edges) can be treated successfully using the effective mass approximation (Stoneham 1975).

'Deep' levels are another matter, and diamond, though a favourite 'model crystal', is difficult in that the band gap is wide $(5.5 \mathrm{eV})$, so that all defects are 'deep'. Nonetheless, a great deal of work has been carried out, and if there is doubt as to which methods of calculation are most appropriate, and disagreements about symmetries of ground states and stabilities and configurations of defects, the calculations have nonetheless provided valuable insights. They have also been handicapped by lack of identification, or in some cases misidentification, of defects. It is not appropriate to discuss the calculations here; for more details, consult Stoneham (1975) and the references therein. However, it does seem that the 'defect molecule' calculations of Coulson and his co-workers successfully predict the energy levels of vacancies in diamond ( $\$ \$ 4.1$ and 4.2$)$.

Some attempts have been made to calculate the one-phonon infrared spectra associated with defects (Dawber and Elliott 1963, Szigeti 1963), particularly the 'nitrogen platelets'. Since it now appears that the platelets do not contain the bulk of the nitrogen these calculations could not be expected to be successful. However, some of the defects have now been correctly identified ( $\$ 3)$, and further calculations are desirable.

One notable result is that the peaks of the defect-activated one-phonon absorption are not expected to coincide exactly with the phonon frequencies of the perfect lattice. If they did, then all defects would produce the same absorption spectrum, 
which would differ only in intensity from one crystal to another (Szigeti 1963); this is obviously not the case ( $\$ \S 1.3$ and 3 ). In fact, for a given defect, the absorption coefficient is given by:

$$
\alpha(\omega)=\frac{2 \pi^{2} D}{3 n c} A^{2}(\omega) S(\omega)
$$

where $D$ is the concentration of impurities, $n$ is the refractive index, $A(\omega)$ is a dipole moment due to the defect and $S(\omega)$ is the density of unperturbed lattice modes.

\subsection{Symmetry lowering perturbations}

The symmetry of a defect is an important clue to its identity. For example, in the diamond lattice any intrinsic defect having tetrahedral symmetry can only be a vacancy or a tetrahedral carbon interstitial. This has been important in confirming the vacancy as the defect responsible for the GR absorption system (see \$4.2.1).

As in atomic spectra, one can in principle study the Stark (electric field) and Zeeman (magnetic field) splitting of zero-phonon lines (Runciman 1969, Kaplyanskii et al 1971). In practice, neither of these techniques has been very successful, largely because zero-phonon lines are relatively broad (typically $>1 \mathrm{meV}$ ), but valuable information can be obtained from magnetic circular dichroism ( $\$ 4.2 .1)$.

The N3 zero-phonon line is the only one known to display a resolvable Stark splitting (Kaplyanskii et al 1970). Other defects have been studied using a modulation technique to resolve splittings within the linewidth (Kaplyanskii et al 1971), but at least some of the results are questionable (see GR1 and H3 in \$4).

Another symmetry determining technique, polarisation of luminescence, has been used on the $\mathrm{H} 3, \mathrm{H} 4$ and $\mathrm{N} 3$ centres. However, it cannot be applied to non-luminescent centres, and some symmetries cannot be distinguished.

The most powerful symmetry determining technique in practice in solids is uniaxial stress, in which the crystal is mechanically compressed along selected symmetry axes. Uniaxial stress has been used to study many centres in diamond. Unlike the Stark and Zeeman effects, resolvable splittings of zero-phonon lines can be obtained with easily achievable stress (Kaplyanskii 1964a, b, 1967, Runciman 1965, Hughes and Runciman 1967).

\section{Infrared spectra (mainly vibrational)}

We have already seen $(\$ 1.3)$ that the different types of diamond can be distinguished by their infrared spectra. In this context 'infrared' means wavelengths greater than $2 \mu \mathrm{m}$, energies less than $0.6 \mathrm{eV}$ or $5000 \mathrm{~cm}^{-1}$. (Other spectra, at about $1 \mu \mathrm{m}$ in the IR, are more conveniently dealt with in $\$ 4$ because they are vibronic, like the visible spectra; most of the spectra considered in $\$ 3$ are vibrational in origin.)

Thus, all diamonds show specimen-independent absorption in the 2-6 $\mu \mathrm{m}$ region (Sutherland et al 1954). This is intrinsic lattice absorption and is due to two- and three-phonon processes, since one-phonon transitions are forbidden by the symmetry of the perfect diamond lattice (Lax and Burstein 1955). Type IIa diamonds show only this infrared absorption. The other types contain impurities which destroy the local symmetry of the lattice and allow one-phonon processes to occur in the 7-13 $\mu \mathrm{m}$ region ( $\mathrm{Ia}$ and $\mathrm{Ib}$ ), or which cause electronic transitions around $0.3 \mathrm{eV}$ (IIb). The 
absorption due to both one- and two-phonon processes reflects the phonon density-ofstates function, modified in the former case by a frequency-dependent factor characteristic of the defect (\$2.4).

\subsection{The lattice bands}

The 2-6 $\mu \mathrm{m}$ bands $\left(1500-5000 \mathrm{~cm}^{-1}\right)$ (figure 4) were recognised as due to the perfect diamond lattice by Sutherland et al (1954), Collins and Fan (1954) and Lax and Burstein (1955). They increase in intensity with temperature, as expected for lattice bands, whereas the defect-activated one-phonon bands in type I diamond do not change. The 3660 and $3120 \mathrm{~cm}^{-1}$ lines are due to three-phonon processes, the others being two-phonon.

In 1970, Solin and Ramdas, using laser-excited Raman spectroscopy plus neutron scattering data, assigned all the features of the infrared and Raman spectra to specific phonons.

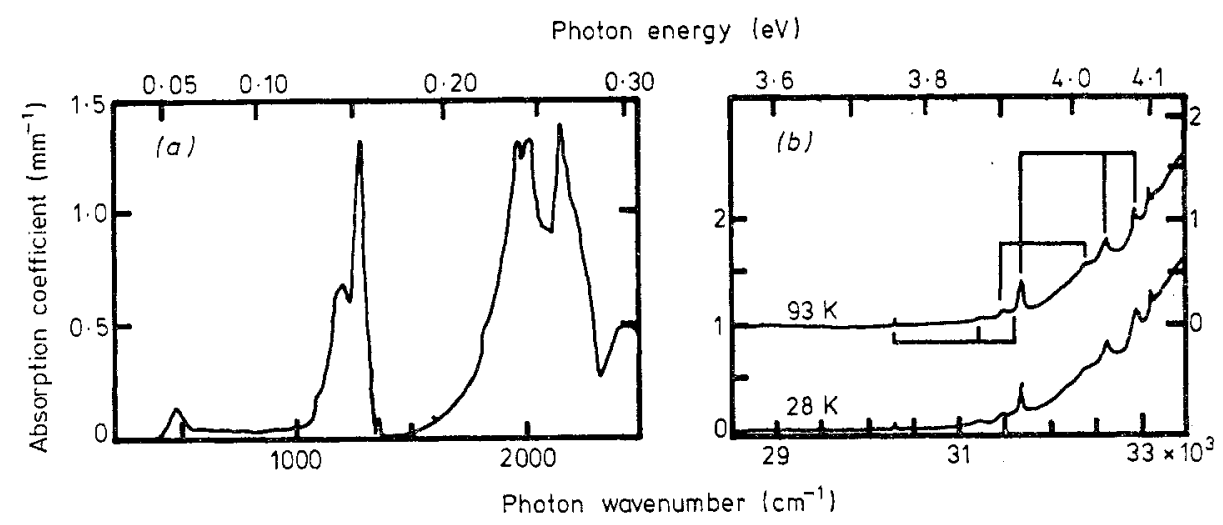

Figure 4. Absorption spectra of a type Ia diamond. (a) Infrared-below $0.2 \mathrm{eV}$ are the impurityinduced group A absorption bands; above $0.2 \mathrm{eV}$ the lattice bands shown by all diamonds. (b) The group A ultraviolet absorption lines and the 'secondary absorption edge'. The true absorption edge is at $5 \cdot 5 \mathrm{eV}$ (after Davies 1976).

\subsection{Defect-induced absorption}

3.2.1. The A, B1 and B2 bands-type Ia diamond. These bands (figures 4 and 5) were discovered by Robertson et al (1934). In 1954 Sutherland et al noted that there were two uncorrelated series, which they labelled $A$ and $B$, plus two independent lines at 1520 and $1540 \mathrm{~cm}^{-1}$. The $B$ bands were subdivided into the independent $B 1$ and B2 groups by Sobolev et al (1968, 1969a, b, c) (table 2), a discovery foreshadowed by Clark (1965) and Charette (1961a), who observed that the $1370 \mathrm{~cm}^{-1}$ peak did not correlate with some of the other B bands. Diamonds with only A bands are common, and consequently the A spectrum is well known. Davies (1971,1972a) has mathematically analysed superimposed A and B bands, and his band B spectra have been experimentally confirmed by Klyuev et al (1970) (figure 5). So we now know the spectra of each of these three series; we will discuss the A, B1 and B2 defects separately.

3.2.1.1. The $A$ defect. Kaiser and Bond (1959), using mass spectrometry and chemical analysis, showed that the absorption coefficient of the most prominent $\mathrm{A}$ 


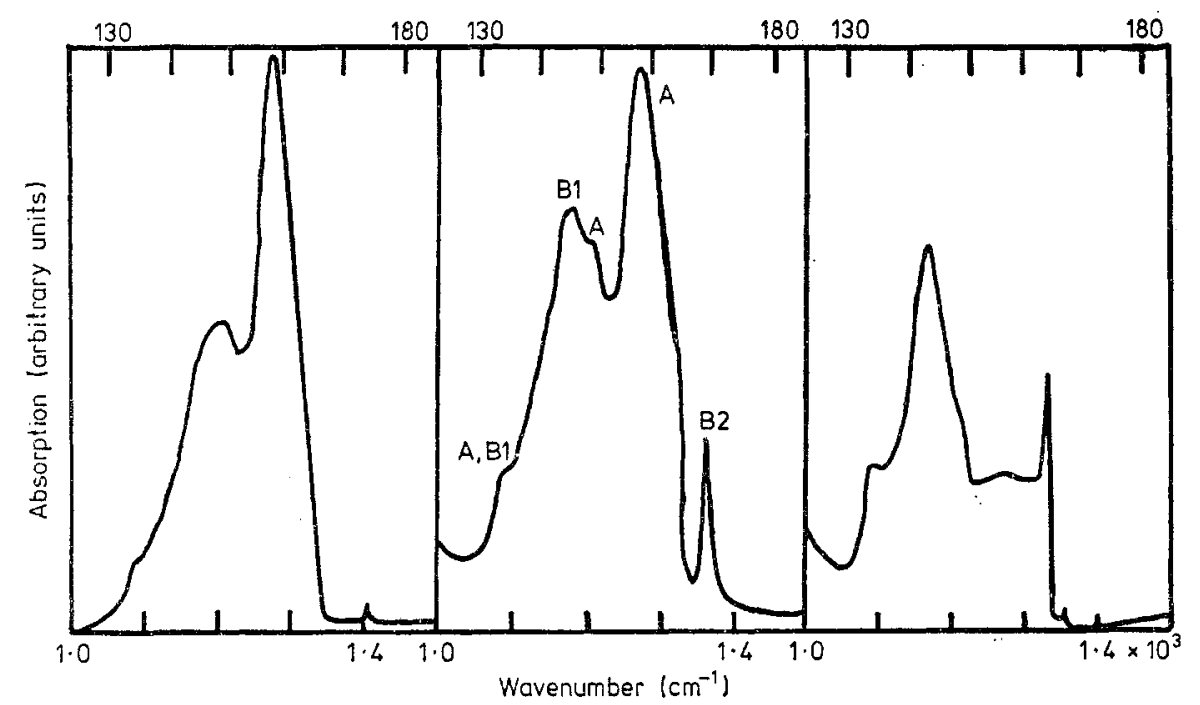

Figure 5. Impurity-induced infrared absorption spectra of three type Ia diamonds: left-the group A lines (type IaA); middle-mixed group A, B1 and B2 lines; right-primarily group B1 lines (type IaB) (after Davies 1971).

peak at $7 \cdot 8 \mu \mathrm{m}\left(1282 \mathrm{~cm}^{-1}\right)$ correlated with nitrogen content of the diamond; this result was confirmed by Lightowlers and Dean (1964) using activation analysis. Both sets of workers reported a correlation between absorption coefficients at $1282 \mathrm{~cm}^{-1}$ and $306.5 \mathrm{~nm}(4.046 \mathrm{eV})$, with $\alpha(306.5)=0.5 \alpha(1282)$. However, according to Summersgill (1972), the true correlation is between the absorption coefficient of the A feature at $1282 \mathrm{~cm}^{-1}$ (i.e. after subtraction of any absorption due to the B1 feature) and the secondary absorption edge coefficient at $306.5 \mathrm{~nm}$, with $\alpha(306.5)=0.65$ $\alpha_{\mathrm{A}}(1282)$. Kaiser and Bond further showed that the $1282 \mathrm{~cm}^{-1}$ peak correlated with the increase in lattice constant of the diamond (see also Lisoivan and Sobolev 1974), but that the density did not vary. This implies that the nitrogen occupies a substitutional position-the greater mass of nitrogen relative to carbon is compensated by the lattice expansion.

It was assumed that this nitrogen gathered in large aggregates ('platelets') which were observable in the electron microscope and caused anomalous features ('spikes') in x-ray Laue photographs. However, in 1968, Sobolev et al showed that the $1282 \mathrm{~cm}^{-1}$ peak correlated neither with the $\mathrm{x}$-ray spike intensity nor with the platelet concentration measured by electron microscopy, but there was a good correlation of spike intensity with the $1370 \mathrm{~cm}^{-1}$ peak, now labelled $\mathrm{B} 2(\$ 3.2 .1 .3)$.

What then was the configuration of the nitrogen responsible for the $1282 \mathrm{~cm}^{-1}$ peak? Sobolev et al assumed it was '.. an association of the type of $\mathrm{N}_{2}$ '. The hypothesis received partial confirmation from Shcherbakova et al (1969), who observed an EPR signal from a pair of nitrogen atoms.

Further confirmation of the nitrogen pairs was provided by Davies (1976), who studied some ultraviolet absorption lines known to be related to the A centre. Although they are not in the infrared it is convenient to discuss them here.

Robertson et al $(1934,1936)$ noted that the diamonds with one-phonon infrared absorption also showed broadband uv absorption above $3 \cdot 7 \mathrm{eV}$ (figure 4). Sutherland 
et al (1954) correlated this 'secondary absorption edge' with the A infrared bands. (The true absorption edge is at $5.5 \mathrm{eV}$.) Clark et al (1956a) discovered that superimposed on the secondary edge were some sharp lines which they labelled N5, N6, N7 and N8 (table 5) that correlated with N5 and with the slope of the secondary edge.

Table 5. The group A absorption lines in the ultraviolet. The defect is trigonal, with an $A$ ground state. Note that the $159 \mathrm{meV}\left(1282 \mathrm{~cm}^{-1}\right)$ phonon is the most prominent in the infrared spectrum also (see table 2 and figures 4 and 5) (after Clark et al 1956a, Davies 1976, Nazaré and Davies 1977).

\begin{tabular}{|c|c|c|c|c|c|c|c|c|c|}
\hline Name & N5 & 一 & - & 一 & N6 & - & N7 & - & N8 \\
\hline Energy $(\mathrm{eV})$ & $3 \cdot 757$ & $3 \cdot 871$ & 3.901 & 3.916 & 3.928 & $4 \cdot 014$ & $4 \cdot 042$ & $4 \cdot 084$ & $4 \cdot 19$ \\
\hline Assignment & $\begin{array}{l}\text { Zero- } \\
\text { phonon } \\
\mathrm{A}-\mathrm{A}\end{array}$ & $\begin{array}{l}\mathrm{N} 5+ \\
113 \\
\mathrm{meV}\end{array}$ & $\begin{array}{l}\text { Zero- } \\
\text { phonon } \\
A-E\end{array}$ & $\begin{array}{l}\mathrm{N} 5+ \\
159 \\
\mathrm{meV}\end{array}$ & $\begin{array}{l}\text { Zero- } \\
\text { phonon } \\
\mathrm{A}-\mathrm{E}\end{array}$ & $\begin{array}{l}3.901+ \\
113 \\
\mathrm{meV}\end{array}$ & $\begin{array}{l}\mathrm{N} 6+ \\
113 \\
\mathrm{meV}\end{array}$ & $\begin{array}{l}\mathrm{N} 6+ \\
159 \\
\mathrm{meV}\end{array}$ & $?$ \\
\hline
\end{tabular}

Davies (1976) and Nazaré and Davies (1977) have discovered and correlated several additional lines not recorded by Clark et al (table 5). N5, N6 and the $3.901 \mathrm{eV}$ peak are zero-phonon lines at a trigonal defect. The others are one-phonon sidebands, the phonon energies being 113 and $159 \mathrm{meV}$. The 'secondary absorption edge' is probably due to internal transitions at the $\mathrm{A}$ centre, rather than to transitions into the conduction band.

Davies shows that these results, together with previous data, severely limit the number of possible models for the A centre. It is a pair of substitutional nitrogen atoms in nearest-neighbour positions (figure $6(a)$ ) -a nitrogen 'molecule'.

The most prominent $A$ infrared band at $159 \mathrm{meV}\left(1282 \mathrm{~cm}^{-1}\right)$ is probably due to the LO phonon at the $\mathrm{L}$ point (the $\langle 111\rangle$ zone boundary), shifted from its perfect lattice value of $1252 \mathrm{~cm}^{-1}$ by the mechanism explained in $\$ 2.4$. Thus the molecular nature of the A centre now seems to be established.

The centre displays the phenomenon of sharp absorption lines superimposed on a continuum like the GR centre. It may therefore be that it displays similarly anomalous photoconductivity behaviour ( $\$ 4.2 .1)$. High-resolution photoconductivity studies of the uv lines are desirable.

What we do not know is why most natural diamonds are type Ia and contain nitrogen pairs, whereas synthetics are usually $\mathrm{Ib}$, containing single nitrogen atoms; nor what role, if any, the A centre plays in forming the 'platelets'. It may be the initial synthesis conditions (Klyuev et al 1973), or the subsequent annealing and pressure treatments (Chrenko et al 1977). Further work is necessary.

3.2.1.2. The group $B 1$ bands. The $\mathrm{B} 1$ spectrum is shown in figure 5 and the wavelengths and energies of the peaks are given in table 2 . There are two questions to consider: the microscopic nature of the B1 centre, and possible correlations with other defects. We will consider the latter first.

Lightowlers and Dean (1964) and Davies and Summersgill (1973) showed that there was no correlation between this centre and N3, but there apparently was a good correlation with $\mathrm{N} 9$, a result also stated or assumed (but not explicitly demonstrated) by many Russian workers (Sobolev and Lisoivan 1972, Sobolev et al 1969a, Klyuev et al 1972). However, Davies and Summersgill (1973), who did demonstrate the $\mathrm{B} 1 / \mathrm{N} 9$ correlation, argued that it was fortuitous, because a typical diamond might 
(a)<smiles>CCNNC</smiles>

(c)<smiles>CNNI</smiles>

(e)

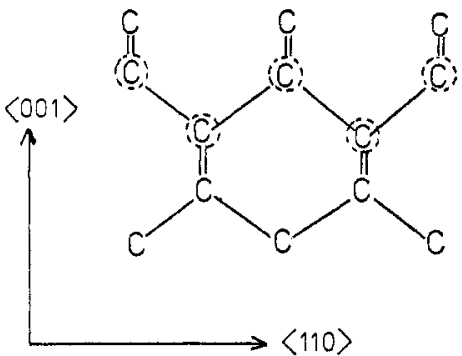

(b)<smiles>CNN[Tl]C</smiles>

(d)<smiles>CNC(=[W])NC</smiles>

(f)<smiles>C=CC1=NN(C)CN(C)N=[Ge]C1C=C</smiles>

Figure 6. The diagram shows a $\{110\}$ plane. (a) The nitrogen pair-thought to be the A aggregate. (b) A vacancy trapped by a nitrogen pair. The defect apparently rearranges to $(c)$ an $\mathrm{N}-\mathrm{V}-\mathrm{N}$ configuration-thought to be the $\mathrm{H} 3$ defect (after Davies 1976). (d) The Mitchell (1964) model of the N3 defect-three nitrogen atoms. $(e)$ The normal diamond lattice. $(f)$ The Lang (1964) model for the platelets. The dotted circles in $(d),(e)$ and $(f)$ indicate atoms lying out of the plane of the paper.

contain an estimated $10^{22} \mathrm{~N} 9$ centres $\mathrm{m}^{-3}$, and more than $10^{24} \mathrm{~B} 1$ centres. The $\mathrm{N} 9$ zero-phonon line, being electronic, is detectable at much lower concentrations than the vibrational infrared bands. This assumes that N9 is an allowed electric dipole transition, which seems likely. We must therefore conclude that the common origin of the $\mathrm{N} 9$ and $\mathrm{B} 1$ bands is not proven.

Davies (1972a) has deduced from the activation analysis results of Lightowlers and Dean (1964) that the B1 centre contains nitrogen. Thus:

$$
3.7 \times 10^{18} K_{1175}>C>1.4 \times 10^{18} K_{1175}
$$

where $C$ is the nitrogen concentration in atoms $\mathrm{cm}^{-3}$ and $K_{1175}$ is the absorption coefficient at $1175 \mathrm{~cm}^{-1}$. Experimentally, Sobolev and Lisoivan (1972) find that:

$$
C=7.6 \times 10^{18} K_{1175}=1.2 \times 10^{18} K_{236.5}
$$

where $K_{236.5}$ is the absorption coefficient due to the N9 defect. The agreement is reasonable, since Davies' result is an estimate. However, the situation is unsatisfactory for several reasons. Firstly, Davies' estimate is based on only one diamond. Secondly, Davies has already rejected the correlation between the B1 and N9 defects which is implicit in the latter equation. Thirdly, Sobolev and Lisoivan give virtually no experimental details. Fourthly, the N9 zero-phonon line width is specimen-dependent, so the integrated absorption should be used, not the peak height. 
Sobolev and Lisoivan (1972) propose their ' $\mathrm{N}_{\mathrm{S}}(111)$ ' model of the B1 centre-a dislocation loop stabilised by nitrogen atoms, the dislocation loops being due to the condensation of vacancies left behind after nitrogen atoms have precipitated into the platelets (Evans and Phaal 1962a). The model is consistent with results reported by Berman and Martinez (1976) and Evans et al (1976), but Klyuev et al (1977) were unable to confirm it, and Evans and Phaal (1962a, b) have observed dislocation loops in type IIa as well as Ia crystals, whereas B1 centres occur only in Ia. The $\mathrm{N}_{\mathbf{S}}(111)$ model has also been proposed to account for $\mathrm{N} 9$ and band $\mathrm{A}(\$ 4.1)$.

What we do know about the B1 defects is that, like B2 centres (platelets) but unlike A centres, they do not affect the lattice constant and they must be very small (Sobolev and Lisoivan 1972). They must have a well-ordered molecule-like structure because they have a well-defined infrared spectrum and give rise to a well-defined vibronic system (H4) after irradiation and annealing (Davies 1972b, Davies and Summersgill 1973). If nitrogen is involved in the B1 centres, the atoms must be present in pairs because no EPR has been detected. They do not contain aluminium (Sobolev et al 1969a, b, c).

In conclusion, the B1 defect has not yet been identified. It is probably a small nitrogen aggregate, possibly associated with dislocation loops, and possibly correlating with the N9 centre. Further work is necessary.

3.2.1.3. The group $B 2$ bands-the platelets. The platelets in type Ia diamonds are interesting because of the length of time they have remained a mystery and because of the wealth of experimental techniques brought to bear on them, though we still do not know their atomic arrangement and how they are formed.

In 1940 Raman and Nilakantan observed anomalous 'spikes' around some of the Laue spots in their $\mathrm{x}$-ray diffraction pictures and it was soon realised that the defects responsible had to be ordered along the $\{100\}$ planes (Hoerni and Wooster 1955, Lonsdale and Smith 1942). Frank (1956) proposed segregated $\{100\}$ planes of impurity atoms, probably silicon, analogous to Guinier-Preston zones in age-hardened alloys, though subsequently Caticha-Ellis and Cochran (1958) found that the silicon concentration in their diamonds was too low to account for the $\mathrm{x}$-ray spikes.

Kaiser and Bond (1959) demonstrated that in type I diamonds the intensity of the group A infrared peak correlated with the concentration of nitrogen impurity. This led Elliott (1960) to speculate that the platelets were composed of nitrogen. Unfortunately, the atomic models he proposed were based on Frank's (1956) calculations of atomic displacements, which were erroneous (Frank 1964). Another model based on nitrogen, and complying with the requirements of valency and of the unchanged density of platelet-containing diamond, was advanced by Lang (1964) (figure 6). Since the platelets had been observed directly by electron microscopy (Evans and Phaal 1962a), the problem appeared to have been solved. The platelets seemed to be nitrogen.

However, there were some discrepancies. Grenville-Wells (1952) and Sutherland et al (1954) showed that the ultraviolet and infrared absorption-known from Kaiser and Bond (1959) to be correlated with nitrogen content (\$3.2.1.1) - did not correlate with the presence or absence of $\mathrm{x}$-ray spikes. The breakthrough came when Sobolev et al (1968) demonstrated that the $\mathrm{x}$-ray spike intensity correlated not with the 'nitrogen' infrared peak at $1282 \mathrm{~cm}^{-1}(\mathrm{~A})$, nor with the $1175 \mathrm{~cm}^{-1}$ (B1) peak, but with the $\mathrm{B} 2$ line at $1370 \mathrm{~cm}^{-1}$. 'The currently popular view that the impurity nitrogen is mostly located in the platelets . . . is erroneous'. Their results were confirmed by 
Evans and Rainey (1975a, b), and Davies (1970a) put an upper limit of 10\% (and no lower limit) on the nitrogen in platelets as opposed to smaller aggregations.

Confirmation of the Russian work came from a totally different techniquethermal conductivity. Turk and Klemens (1974), analysing the thermal conductivity variation with temperature of one diamond, deduced that $97 \%$ of the nitrogen it contained was in small 'point-like' aggregates, and only $3 \%$ in the planar platelets. Their results were confirmed by Berman et al (1975) and Berman and Martinez (1976).

We thus have a puzzle. Platelets are found only in type Ia (i.e. nitrogen-containing) diamonds; nitrogen is the only impurity known to be present in sufficient concentrations to account for the platelets; yet the platelets contain little if any nitrogen. Since the $1370 \mathrm{~cm}^{-1}$ platelet peak has never been observed without the $A$ and/or $B$ features it appears that nitrogen is a necessary but not sufficient condition for platelet formation. Evans (1973) therefore proposed that the platelets are primarily carbon; they are formed during growth by interstitial nitrogen atoms pairing with substitutional nitrogens to give a substitutional nitrogen pair and an interstitial carbon atom. The carbon interstitials then aggregate into platelets.

Some support for this hypothesis came from Berman et al (1975) and Berman and Martinez (1976). Their platelet concentration as measured by thermal conductivity was in rather better agreement with that deduced from the $1370 \mathrm{~cm}^{-1}$ peak if it was assumed that the platelets were stacking faults rather than nitrogen aggregates. Additional support came from the electron microscopy studies of Woods (1976), who studied 'giant' platelets up to $5 \mu \mathrm{m}$ long (the usual size is $0.1 \mu \mathrm{m}$ or less). He concluded that they were interstitial layers of carbon. However, his conclusions have been questioned by Lang (1977). Other workers (Bursill et al 1977), using phase contrast electron microscopy, have found that the Lang (1964) nitrogen platelet model (figure 6) fits their results best. Hence the question is still open. The answer may come from high-temperature high-pressure annealing studies (Nikitin et al 1969, Klyuev et al 1972, Evans et al 1976, Chrenko et al 1977).

The only optical property of the platelets that we have been concerned with so far is the absorption peak at $1370 \mathrm{~cm}^{-1}$. However, it has recently become apparent that platelets give rise to visible and infrared luminescence. In 1971 Wight et al found a correlation between a broad featureless cathodoluminescent band peaking at $1.25 \mathrm{eV}$ and the integrated intensity of the $1370 \mathrm{~cm}^{-1}$ peak. Hanley et al (1977) and Kiflawi and Lang (1977) have observed this luminescence from individual 'giant' platelets (figure 7 (plate)) and have shown that it is highly polarised (as one would expect from a planar aggregate). It is not clear whether this luminescence is intrinsic to the platelets or is due to decoration by other defects, though the former seems more likely since the luminescence is apparently not observed independently of the platelets.

Visible cathodoluminescence also occurs due to decoration of the platelets by $\mathrm{H} 3$ defects (Hanley et al 1977). Whether the H3 defects, which are thought to be a vacancy trapped by an $A$ nitrogen pair, have migrated to the platelets or whether they are an intrinsic part of the structure, is not clear. It may be significant that both the Lang (1964) model of the platelets and the Davies (1976) model of the H3 centre involve a pair of nitrogen atoms (Walker 1977a).

3.2.2. The single nitrogen substitutional centre-type $I b$ diamond. This defect was first observed by Smith et al (1959) using paramagnetic resonance. Nitrogen could be unambiguously identified because ${ }^{14} \mathrm{~N}$, the most common isotope $(99.6 \%$ abundant), is virtually the only nuclide with unit nuclear spin, and because nitrogen is a 

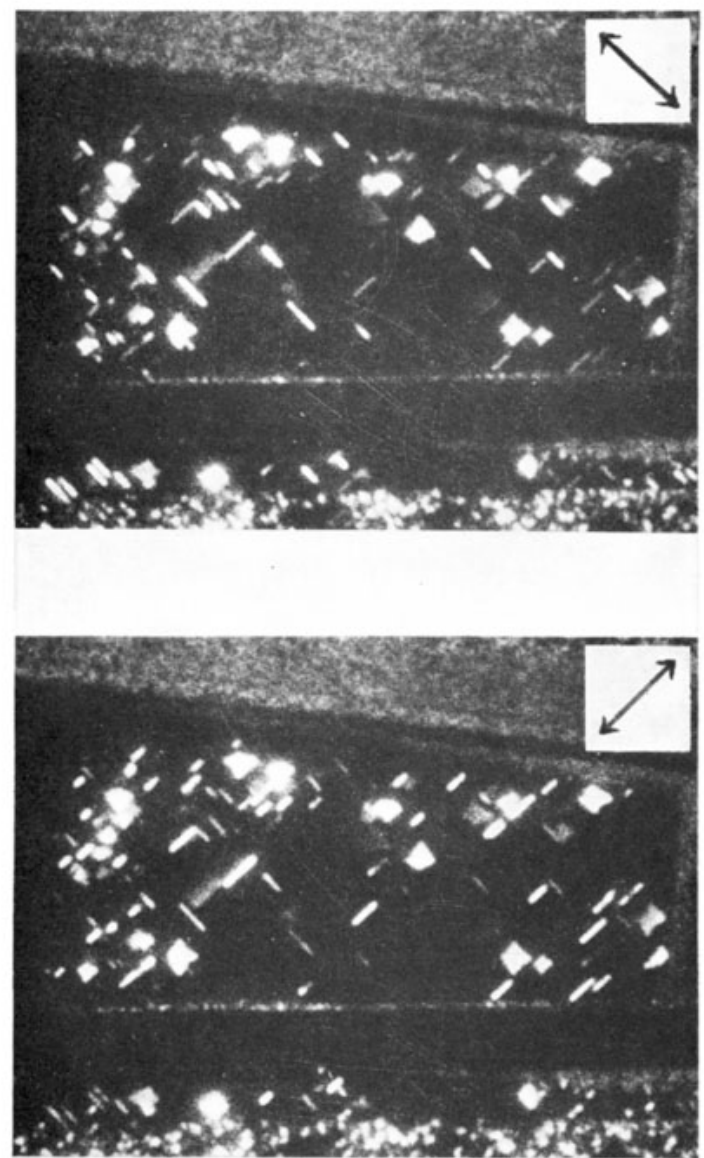

Figure 7. Polarised infrared $(1.25 \mathrm{eV})$ cathodoluminescence from 'giant' $\{001\}$ platelets. The arrows, length $100 \mu \mathrm{m}$, are parallel to $E$ vector. The rectangles are platelets in the plane of the diagram; the lines are 'edge-on' platelets. Smaller platelets can be seen along the lower margins of the photographs (after Kiflawi and Lang 1977). 
common impurity in diamond. The nitrogen atom substitutes for a carbon lattice atom, and the paramagnetic electron is located in an antibonding orbital between the nitrogen and one of the nearest-neighbour carbon atoms. The defect thus has $\langle 111\rangle$ (trigonal) symmetry.

About $0.1 \%$ of all clear natural diamonds contain a homogeneous distribution of paramagnetic nitrogen and show characteristic optical absorption. This was discovered by Dyer et al (1965), who called them type Ib to distinguish them from the diamonds containing nitrogen aggregates $(\$ \S 1.3$ and 3.2.1). Type Ib crystals are yellow or green, depending on their nitrogen concentration. Dyer et al showed that they display one-phonon absorption in the infrared (figure 8) and a continuum starting at about $2 \mathrm{eV}(600 \mathrm{~nm})$ and increasing in intensity towards the ultraviolet. There are sharp lines on the continuum at $4 \cdot 07,4 \cdot 15,4 \cdot 24,4.58$ and $4.64 \mathrm{eV}$, which are resolved only at $80 \mathrm{~K}$, and a broad $(0.5 \mathrm{eV})$ peak at $4.4 \mathrm{eV}(280 \mathrm{~nm})$ which could be their phonon band, as well as other broad peaks below $4 \mathrm{eV}$. The integrated intensity of the EPR line correlates with the integrated intensity of the infrared band and with the slope of the absorption at $2 \cdot 6 \mathrm{eV}$.

Although natural type Ib diamonds are rare, synthetics usually fall into this category unless nitrogen is deliberately excluded from the reaction or other dopants added ( $\$ 1.7)$. The spectra of Dyer et al have been confirmed by work on synthetic crystals (Charette 1961b, Sobolev et al 1969c, Chrenko et al 1971, Klyuev et al 1975a). There is only order-of-magnitude agreement on the relative strengths of the EPR and optical spectra. An absorption coefficient of $1 \mathrm{~cm}^{-1}$ at $1130 \mathrm{~cm}^{-1}$ is produced by $0.8 \times 10^{24}$ atoms $\mathrm{m}^{-3}$ of nitrogen (Sobolev et al 1969c, Klyuev et al 1975a) or by $4 \times 10^{24}$ atoms $\mathrm{m}^{-3}$ (Chrenko et al 1971). The latter used larger crystals, which implies greater reliability, but independent results, preferably using natural crystals, are desirable.

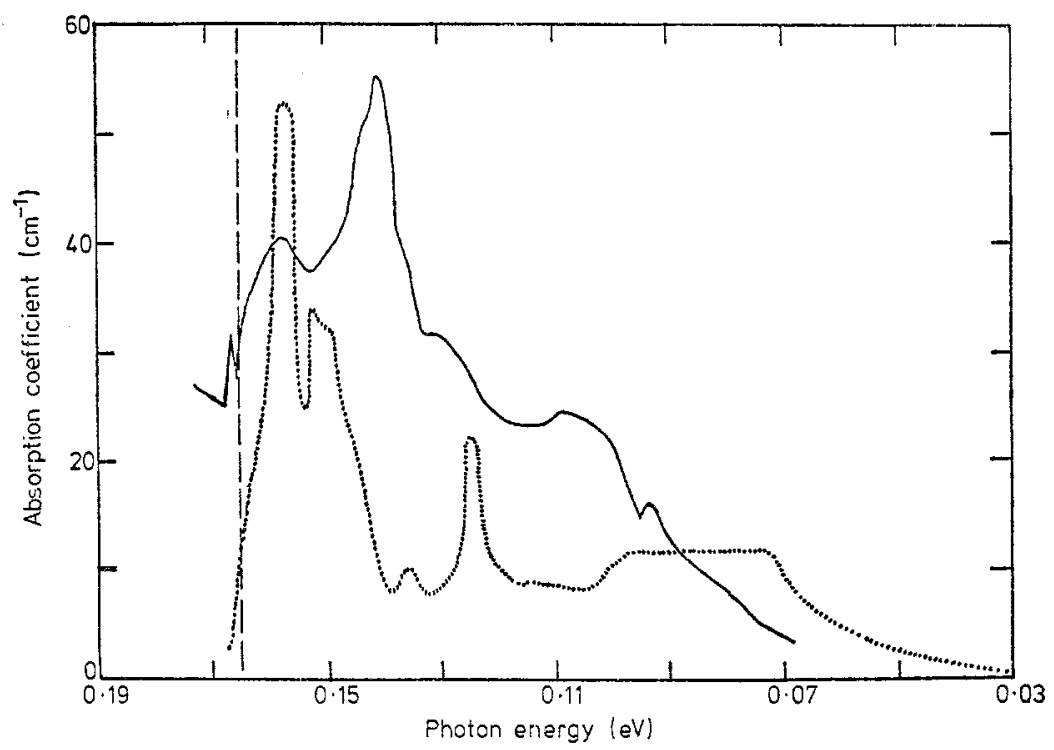

Figure 8. Full curve: infrared absorption spectrum of a type Ib diamond; dotted curve: calculated density of states (after Angress et al 1968). The peaks at 93 and $105 \mathrm{meV}$ are as yet unconfirmed by other workers; Davies (1977a) has suggested that the $167 \mathrm{meV}$ peak is an independent feature. 
To investigate further the properties of nitrogen in diamond, some synthetics have been doped with ${ }^{15} \mathrm{~N}\left(I=\frac{1}{2}\right)$. The earliest attempt (Cannon 1971) had limited success in that some changes were produced in the EPR spectrum. Later workers (Klyuev et al 1975b, Samoilovich et al 1975) measured the hyperfine parameters of $15 \mathrm{~N}$, which were in excellent agreement with the calculated values. Furthermore, there was a change in the infrared spectrum. The $1135 \mathrm{~cm}^{-1}$ peak had shifted to $1120 \mathrm{~cm}^{-1}$. No changes were observed in the other IR peaks. This suggests that the $1120 \mathrm{~cm}^{-1}$ peak is an 'in-band resonance', due to a vibration characteristic of the defect but falling within the range of diamond lattice vibrations because nitrogen has a higher mass than carbon (as opposed to a 'local mode' due to a lighter atom which would lie above the maximum lattice frequency - see Dawber and Elliott (1963)). The other peaks, since they do not change, are presumably due to perfect lattice phonons. Their energies will not coincide exactly with those of the perfect lattice $(\$ 2.4)$.

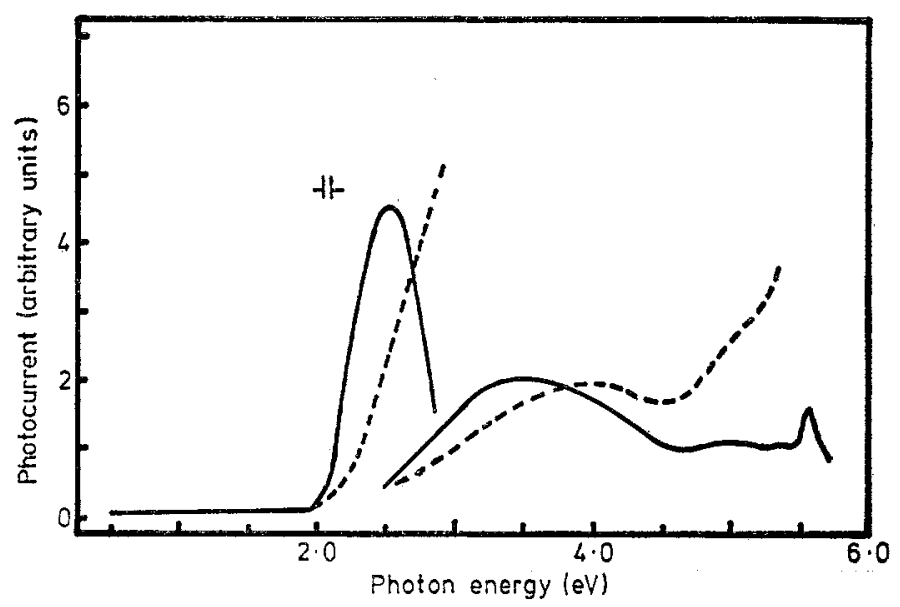

Figure 9. The room-temperature photoconduction spectrum of a synthetic type Ib diamond. Full curve: uncorrected; broken curve: corrected for intensity of exciting sources (after Farrer 1974). Two light sources were used.

Nitrogen, being a group $\mathrm{V}$ element, should be a donor in diamond, and experiments on type Ib crystals confirm this. Optical and thermal measurements indicate that the donor level is $2 \mathrm{eV}$ below the conduction band (figure 9) and the thermoelectric effect indicates n-type conductivity (Farrer 1974, Vermeulen and Farrer 1975). (In fact, the thermal activation energy is $1.7 \mathrm{eV}$, whereas the optical threshold is at $2 \cdot 2 \mathrm{eV}$. The difference is a consequence of the Jahn-Teller effect and is close to the value of $0.7 \mathrm{eV}$ deduced for the Jahn-Teller energy from EPR measurements (Shul'man et al 1967, Loubser and Van Ryneveld 1967).) It seems that the surmise by Dyer et al that the UV absorption is due to transitions into the conduction band is correct. Earlier work had placed the donor level at $4.05 \mathrm{eV}$ below the conduction band (Dean 1965, Denham et al 1967). This level needs further investigation, but it is not the single nitrogen donor.

Thus, the nitrogen substitutional centre in diamond appears to be well-characterised, both from the EPR and optical points of view. Further work is desirable, particularly on the Uv peaks; they may be another example of anomalously sharp lines on a photoconduction continuum, like the GR2-8 lines. 
3.2.3. The boron acceptor centre-type IIb diamond. There have been several recent reviews of the electrical and optical properties of type IIb diamonds which are p-type semiconductors (Vavilov 1975, Vavilov and Konorova 1976, Bourgoin and Walker 1976, Davies 1977a, Collins and Lightowlers 1978). The acceptor impurity was originally thought to be aluminium, but it is now known to be boron (Chrenko 1973, Lightowlers and Collins 1976).

Electrical measurements (Wedepohl 1957a) show that type IIb diamonds are normal p-type semiconductors. The ground state of the acceptor centre is about $0.37 \mathrm{eV}$ above the valence band, and the Hall mobility is $0.1-0.15 \mathrm{~m}^{2} \mathrm{~V}^{-1} \mathrm{~s}^{-1}$ at room temperature. Unrealistic values of the hole effective mass are obtained unless it is assumed that some of the acceptor centres are compensated for by donors. Typical concentrations in natural diamonds are $10^{22}-10^{23}$ acceptors $\mathrm{m}^{-3}$ and $10^{21}-10^{22}$ donors $\mathrm{m}^{-3}$. At larger concentrations $\left(10^{24} \mathrm{~m}^{-3}\right)$ and low temperatures $(<200 \mathrm{~K})$, where holes cannot be excited into the valence band, conduction can occur by the hopping of carriers from occupied to unoccupied boron sites (Williams et al 1970, Bourgoin and Walker 1976). In such a case the activation energy for conduction will be much lower -typically $0.2 \mathrm{eV}$.

Wedepohl $(1957 \mathrm{a}, \mathrm{b})$ established that the acceptor centre displayed absorption peaks at $307,346,363,514,537$ and $675 \mathrm{meV}$ which correlated in intensity with each other, with a continuum absorption starting at $370 \mathrm{meV}$ and extending to $2 \mathrm{eV}$, and with the number of acceptors uncompensated by donors $\left(N_{\mathrm{A}}-N_{\mathrm{D}}\right)$. The three lower-energy peaks were recognised as bound state transitions, and the continuum as ionisation of the centre leading to photoconductivity (process 1 of figure 12).

The $307 \mathrm{meV}$ peak is kept broad by a lifetime effect due to the proximity of a nearby level at $266 \mathrm{meV}$ (Smith and Taylor 1962). Transitions to this level from the ground state are forbidden, but are observed in highly-doped synthetic crystals (Collins et al 1965) and in photoconductivity (Collins et al 1969). Below $300 \mathrm{~K}$ the 346 and $363 \mathrm{meV}$ peaks show fine structure (figure 10), some of which disappears at $4 \mathrm{~K}$; this was explained by Crowther et al (1967) using effective mass theory (figure 11). Their calculations were confirmed by their own uniaxial stress work and by the Zeeman and Stark effect measurements of Bagguley et al (1966) and Anastassakis (1969).

The broader absorption peaks at 514, 537 and $675 \mathrm{meV}$ which are above the ionisation energy $(370 \mathrm{meV})$, plus others at 462 and $625 \mathrm{meV}$, are phonon replicas of the lower-energy peaks, the phonon energy being approximately $160 \mathrm{meV}$ (Smith and Taylor 1962). Hence photons with energies greater than $370 \mathrm{meV}$ can be absorbed without creating free carriers (process 2 of figure 12). Since this is competitive with direct ionisation (process 1 of figure 12), the photoconductivity spectrum ought to show troughs at these absorption peaks, which is indeed the case (Hardy et al 1962, Hardy 1962a, Collins and Lightowlers 1968, Lightowlers et al 1968).

The photoconductivity spectrum also shows dips which do not correspond to absorption peaks. They are due to the capture of free holes into excited states of the acceptor centre with emission of phonons (process 3 of figure 12) (Hardy et al 1962). Later work (Collins and Lightowlers 1968, Lightowlers et al 1968, Collins et al 1969), taking measurements up to $2.6 \mathrm{eV}$, showed that up to 14 phonons could be emitted by a hole before it was captured by the 304 and $348 \mathrm{meV}$ excited states. Furthermore, holes could be captured by excited states to which transitions from the ground state are forbidden. This confirmed the existence of the level at $266 \mathrm{meV}$, which was mentioned earlier, plus others at 240 and $289 \mathrm{meV}$. 


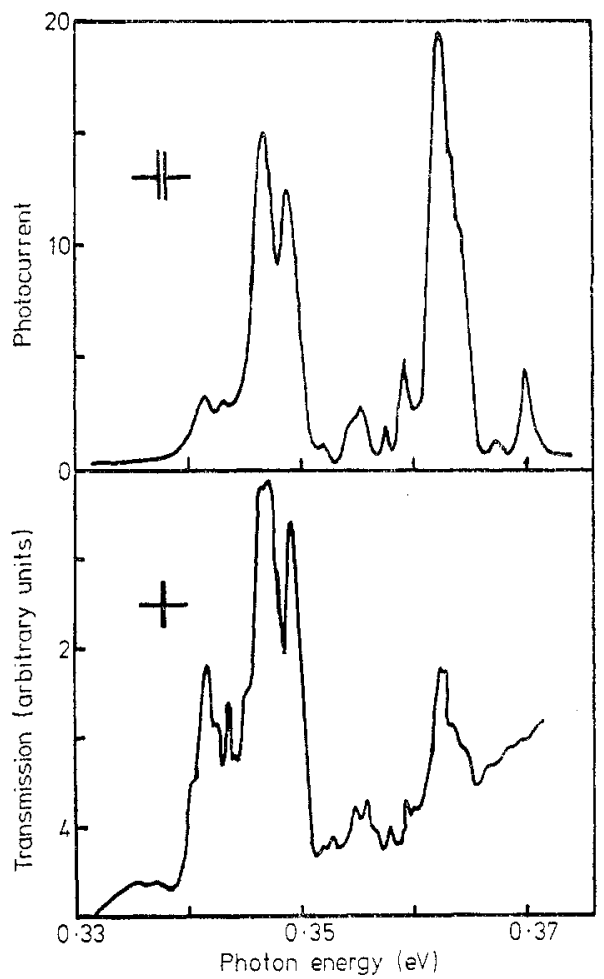

Figure 10. Comparison of photoconductivity at $120 \mathrm{~K}$ (upper curve) with absorption at $80 \mathrm{~K}$ (lower curve) of two natural IIb diamonds. Note that the photoconductivity peaks nearest to the ionisation energy $(0.37 \mathrm{eV})$ are enhanced relative to the absorption peaks, due to photothermal ionisation (process 4 of figure 12) (after Collins and Lightowlers 1968).

There is yet another photon/phonon process observed in photoconductivity at the acceptor centre-photothermal ionisation-in which, unlike the other two processes, absorption peaks coincide with photoconductivity peaks (figure 10). It was observed and explained by Collins and Lightowlers (1968) and Lightowlers et al (1968) and is shown as process 4 of figure 12. Holes are raised to the bound excited states, and the lifetime is sufficient for them to then be thermally excited into the conduction band. In photoconductivity the peaks at $364 \mathrm{meV}$ are enhanced relative to the $348 \mathrm{meV}$ peaks because less thermal energy is necessary to ionise them. The activation energies deduced from the photothermal process $(24.5 \mathrm{meV}$ for the $348 \mathrm{meV}$ peaks and $8.5 \mathrm{meV}$ for the $364 \mathrm{meV}$ peaks) tie in well with the thermal ionisation energy from the ground state of $373 \mathrm{meV}$ (Lightowlers and Collins 1966). The photoconductivity peaks are usually better resolved than the absorption peaks because unperturbed excited states, having longer lifetimes, are most likely subsequently to undergo thermal ionisation.

At $5 \mathrm{~K}$ there is not enough energy for the photothermal ionisation mechanism to operate. Nonetheless, weak photoconductivity peaks are observed, and they are dramatically enhanced if the crystal is irradiated with 'bias light' of energy greater than $370 \mathrm{meV}$ (i.e. within the ionisation continuum). The photocurrent is due to tunnelling of holes between excited levels of adjacent acceptors. The bias light, by ionising centres, provides vacant acceptor sites to which holes can tunnel from occupied sites (Collins and Lightowlers 1968, Lightowlers et al 1968). 
(a)

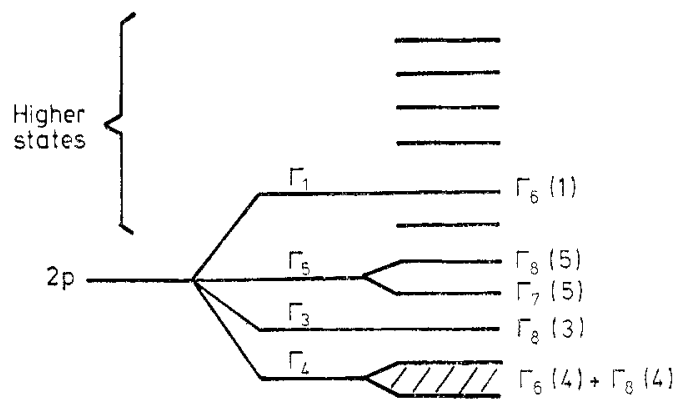

2s

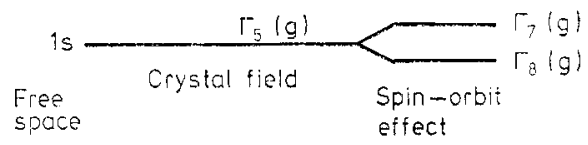

(b)
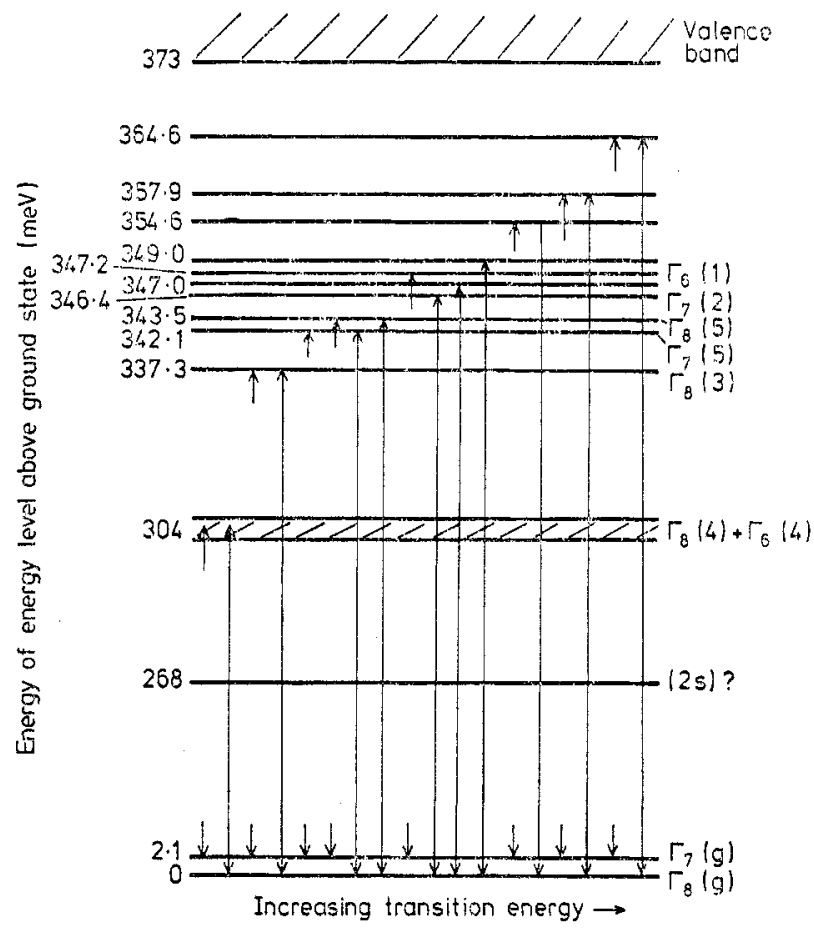

Figure 11. (a) A schematic diagram showing the effect of the diamond crystal field and the spin-orbit interaction on the boron atom. The bracketed characters indicate the parentage of the levels (see Crowther et al 1967, Anastassakis 1969). (b) The energy levels of the boron acceptor in diamond. All transitions are allowed excepted $\Gamma_{6} \rightarrow \Gamma_{6}$ and $\Gamma_{7} \rightarrow \Gamma_{7}$, and virtually all are observed (arrows). The unlabelled levels arise from more highly excited states than $2 \mathrm{p}$. The diagram is based on table I of Crowther et al (1967). The levels at 347,349 and 364.6 are thought to be four-, two- and four-fold degenerate, respectively (Anastassalkis 1969, Bagguley et al 1966). Transitions to the level at $268 \mathrm{meV}$ (and to others at 240 and $289 \mathrm{meV}$ ) are forbidden from the ground state (Collins and Lightowlers 1968, Collins et al 1969). The split ground state explains why some of the acceptor transitions disappear at low temperature (these transitions are shown as incomplete lines). 


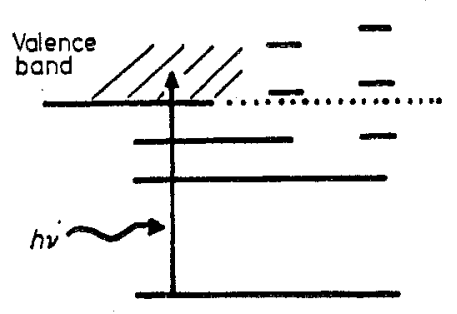

(1)

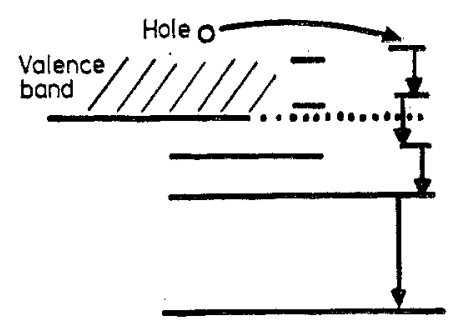

(3)

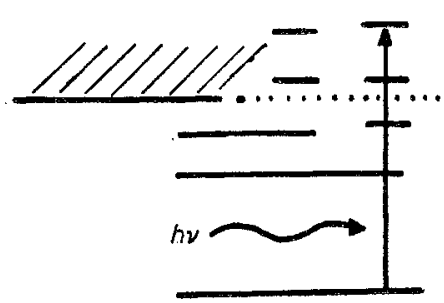

(2)

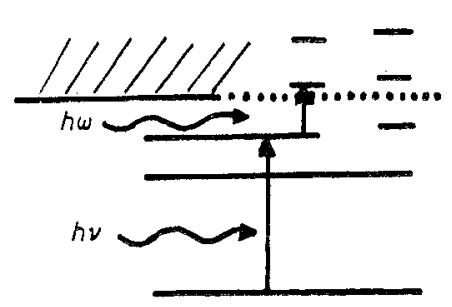

(4)

Figure 12. Photoprocesses at the acceptor centre: $h v$ and $h \omega$ indicate photon and phonon energies, respectively. (1) absorption leading to photoconductivity-photon absorbed, free hole created. (2) Absorption without photoconductivity-photon excites higher vibronic levels. (3) Hole capture into higher vibronic levels causing photoconductivity minima. (4) Photothermal ionisation-absorption of photon plus phonon leads to photoconductivity.

Another unusual feature of the acceptor centre is a weak absorption band at $160 \mathrm{meV}$ first recorded by Charette (1961a) which Smith and Taylor (1962) studied in detail. It sharpens considerably on going from $293 \mathrm{~K}$ to $85 \mathrm{~K}$, and displays structure reminiscent of the $\mathrm{A}$ and $\mathrm{B}$ infrared bands due to nitrogen. Smith and Taylor proposed that it had the same origin; a normally forbidden one-phonon process activated by the impurity. However, it differs significantly in two respects from the A and B bands. Firstly, the A and B bands change very little with temperature. Secondly, the acceptor centre is very efficient in inducing single-phonon absorption. Only $3 \times 10^{22}$ acceptors $\mathrm{m}^{-3}$ are needed to give a $1 \mathrm{~cm}^{-1}$ absorption coefficient, compared with $7 \times 10^{25}$ nitrogen atoms or radiation defects. This is a consequence of coupling between the electronic and nuclear motions (Hardy 1962b, Davies 1977a).

Free carrier absorption in semiconducting diamond is seen in the infrared at wavelengths above $10 \mu \mathrm{m}(<0.1 \mathrm{eV})$, particularly if the diamonds are heated to populate the valence band with holes (Clark et al 1960).

The identity of the donor which compensates some of the acceptor centres is currently unknown. The question is discussed by Collins and Lightowlers (1978), Davies (1977a) and Collins (1978).

Wedepohl (1957b) discovered that irradiation of semiconducting diamonds decreased their conductivity, hole concentration and hole mobility (see also Clark et al 1961). The infrared peaks were correspondingly reduced in intensity. His 
conclusion was that the irradiation introduced extra donors (see also Dyer and Ferdinando (1966) and \$4.2.1). Wedepohl discounted the possibility that the acceptors were displaced from their lattice sites, because both the irradiation dose and the acceptor concentration were small; this would rule out a direct collision process.

However, we now know that in silicon the aluminium acceptors can be ejected from the substitutional sites by migrating silicon interstitials (Watkins 1965). In diamond, most authors follow Wedepohl and assume, explicitly or otherwise, that the number of acceptors stays constant during irradiation. In the light of the evidence from silicon it would be advisable to re-examine this assumption. It could account for difficulties in interpretation experienced by Wedepohl and others (see also $\$ 4.2 .7$ for a defect which might involve a carbon or boron interstitial).

Thus we have seen that the acceptor centre in diamond displays many interesting physical effects. Some of these are qualitatively different from acceptors in silicon or germanium, because the depth of the acceptor level in diamond is significantly greater than the phonon energy. Scope for further work lies not so much with the acceptor centre itself (though calculations might be made on the higher excited states, extending the work of Crowther et al) as with irradiation of boron-doped synthetic crystals in order to identify impurity-related defects. Other areas of interest are the possible production of devices if n-type diamond can be synthesised, and the elucidation of whether elements other than boron can form acceptor centres.

3.2.4. Irradiation-induced absorption. Neutron or electron irradiation induces single-phonon infrared absorption similar in character to the A and B bands. Peaks are observed at 148,139 and $125 \mathrm{meV}$, with weaker maxima at 165 and $159 \mathrm{meV}$, and a line above the Raman energy at $190 \mathrm{meV}$ (compare table 2 and figures 4 and 5). The absorption coefficient per defect is much the same for radiation damage as it is for nitrogen. Annealing at $1000 \mathrm{~K}$ considerably reduces the absorption, as it does the visible spectra induced by irradiation (Smith and Hardy 1960, Smith 1961, Smith et al 1962, Evans and Wild 1967).

\section{Visible and near-visible spectra}

This section deals with electronic and vibronic spectra from $0.8 \mathrm{eV}(1500 \mathrm{~nm})$ to the fundamental absorption edge at $5.5 \mathrm{eV}(220 \mathrm{~nm})$. Energies and wavelengths quoted in this section will usually refer to zero-phonon lines. It is convenient to subdivide spectra into those present naturally, those created by irradiation, and those induced by subsequent annealing. Lack of space has precluded the discussion of several defects (see Davies 1977a).

\subsection{Unirradiated diamond}

4.1.1. The $\mathrm{N} 3$ centre $(415 \mathrm{~nm}, 2.99 \mathrm{eV})$. The N3 absorption and photoluminescence is predominant in many natural diamonds, which has ensured that this centre has been much studied. It has previously been discussed by Clark (1965), Davies (1972a, 1977a) and Davies and Summersgill (1973).

The N3 spectra are shown in figure 13 and are an excellent example of mirrorimage vibronic spectra. Dyer and Matthews (1957) found the centre in all the type Ia and IIa diamonds they studied, though not in type IIb. N3 correlates in intensity 

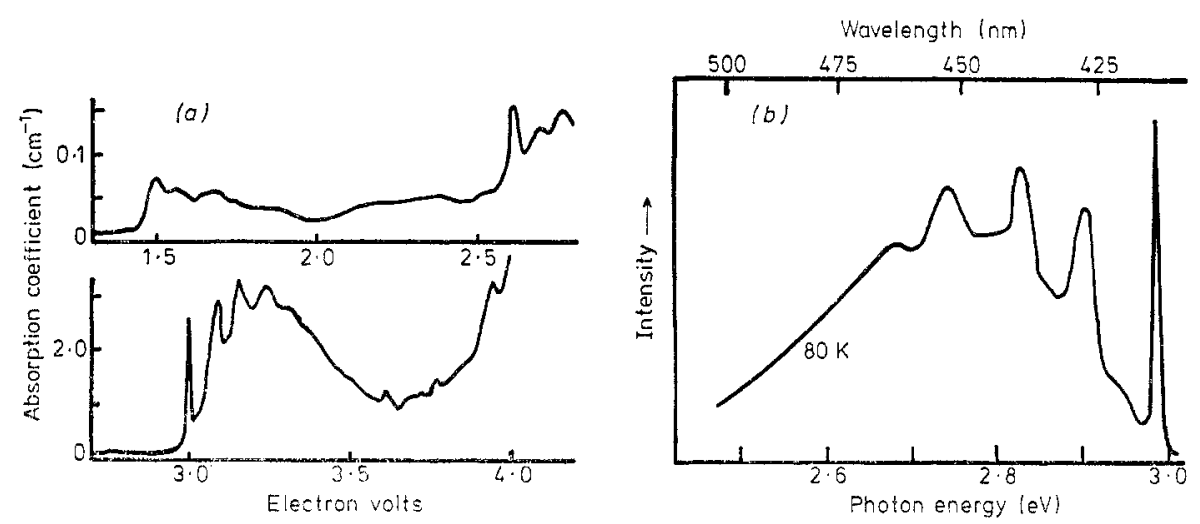

Figure 13. (a) N3 absorption spectrum showing also N1, N2 and N4 (after Clark et al 1956a). (b) N3 photoluminescence spectrum (after Dyer and Matthews 1957). Note the almost perfect mirror symmetry of the $\mathrm{N} 3$ absorption and luminescence spectra.

with N2 $(477.6 \mathrm{~nm}, 2.596 \mathrm{eV})$ and N4 $(344.2 \mathrm{~nm}, 3.603 \mathrm{eV})$, but not with N5 to N8. $\mathrm{N} 1(825 \mathrm{~nm}, 1.50 \mathrm{eV})$ is weak and has not been correlated with the other lines (Clark et al 1956a). Suggestions that N3 correlates with N9 (Raal 1959) and the group B infrared lines (Sutherland et al 1954) have been refuted by Lightowlers and Dean (1964), Dean and Male (1964b), Sobolev et al (1970) and Davies and Summersgill (1973). A luminescence line at $2.001 \mathrm{eV}$ may be a transition at the $\mathrm{N} 3$ centre (Dyer and Matthews 1957, Clark 1965).

The N3 absorption and luminescence bands have been studied in detail by Sobolev et al (1969b), Nedzvetskii and Dymke (1970) and Nedzvetskii and Gaisin (1974a), who have assigned all the phonon peaks using neutron scattering data and have shown that departures from mirror symmetry are small. Coupling is predominantly to symmetry-maintaining distortions along the trigonal axis; there is no evidence of Jahn-Teller effects (Davies 1974b).

Polarised luminescence and uniaxial stress agree in assigning N3 to a transition from an A ground state to an $\mathrm{E}$ excited state at a trigonal defect (Clark et al 1962, Clark 1965, Runciman 1965, Crowther and Dean 1967, Clark and Norris 1970). There is a small splitting of $0.59 \mathrm{meV}$ in the excited state, presumably due to the spin-orbit effect (Davies and Summersgill 1973). (This is not to be confused with the variable splitting observed in luminescence by some authors and explained as self-absorption by Dyer and Matthews (1957).) The Stark effect rules out the trigonal point groups $\mathrm{D}_{3 \mathrm{~d}}$ and $\mathrm{D}_{3}$, and hence $\mathrm{N} 3$ must have $\mathrm{C}_{3 \mathrm{v}}$ symmetry. It is the only defect in diamond to display a resolvable Stark effect in a static electric field (Kaplyanskii et al 1970, 1971).

The excitation spectrum of $\mathrm{N} 3$ contains a peak corresponding to N4, and depolarisation of the N3 luminescence is observed when N4 is excited. This is explained if $\mathrm{N} 4$ is an A to A transition ('a $\pi\langle 111\rangle$ oscillator') at the same centre, and if the N4 excited state decays, radiatively or otherwise, by a transition to the N3 excited state (Clark and Norris 1970). Hence there should be no luminescence counterpart of the N4 absorption, as Dean (1965) found. This implies that N4 is a forbidden transition. Consistent with this, Welbourn and Davies (1977) have noted that both N2 and N4 are broad (typically $215 \mathrm{~cm}^{-1}$ compared to $30 \mathrm{~cm}^{-1}$ for N3). They suggest that these lines are vibronic in origin, the zero-phonon lines being forbidden.

Thus the N3 energy level diagram appears to be as shown in figure 14. It suggests 


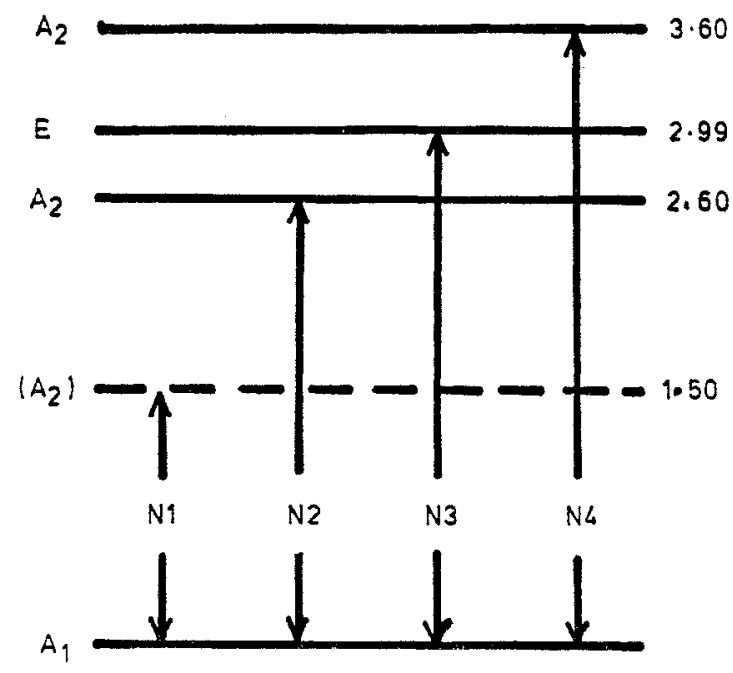

Figure 14. The $N 3$ energy level diagram. The ground state is assumed to be $A_{1}$ (if it is $A_{2}$ then subscripts 1 and 2 are interchanged). The only forbidden transition in $\mathrm{C}_{3 \mathrm{v}}$ is $\mathrm{A}_{1}-\mathrm{A}_{2}$. The $\mathrm{N} 1$ level has not been confirmed as belonging to the centre. It is assumed that $\mathrm{N} 2$ and $\mathrm{N} 4$ are forbidden transitions.

that transitions between the various excited states should be observable in luminescence, at energies between 0.4 and $2.1 \mathrm{eV}$. Many unidentified lines have been observed in this region, including the one at $2.001 \mathrm{eV}$ already mentioned, though none have yet been correlated with $\mathrm{N} 3$. The diagram may also explain why $\mathrm{N} 3$ is not usually observed in cathodoluminescence; the most-favoured decay path for the excited state may not include $E \rightarrow A_{1}$. Dean's (1965) model for the N3 centre now seems unlikely (Collins 1974). An alternative model, proposed by Mitchell (1964), consists of three nitrogen atoms in next-nearest-neighbour positions, bonded to a common carbon atom (figure 6 ). This has the requisite $\mathrm{C}_{3 \mathrm{v}}$ symmetry and is consistent with $\mathrm{N} 3$ being found in nitrogen-rich diamonds. Other evidence relating this model to N3 comes from paramagnetic resonance (Welbourn and Davies 1977, Loubser and Wright 1973a, Loubser 1977), though further work is necessary to clarify certain discrepancies.

Until recently the N3 system was seen only in natural diamonds; now Chrenko et al (1977) have demonstrated that it can be induced in synthetic Ib diamonds by suitable heat and pressure treatment.

4.1.2. The $N 9$ centre ( $236 \mathrm{~nm}, 5 \cdot 26 \mathrm{eV}$ ). N9 was christened by Raal (1959), who also refers to earlier work. He studied the centre in absorption in very thin samples-it is often obscured by the 'secondary absorption edge' due to the A nitrogen aggregate. N9 luminescence can be found in most Ia and IIa diamonds, though not in types Ib and IIb (Dean and Male 1964a,b, Lightowlers and Dean 1964, Wight and Dean 1967, Crowther and Dean 1967).

Raal proposed that N9 correlated with N3; this has been disproved by various authors (see \$4.1). As we have already seen in \$3.2.1.2, some authors claim a correlation between N9 and the B1 infrared bands, though the evidence is conflicting and the relationship doubtful.

In absorption the N9 lines are often broad; in luminescence and luminescence 


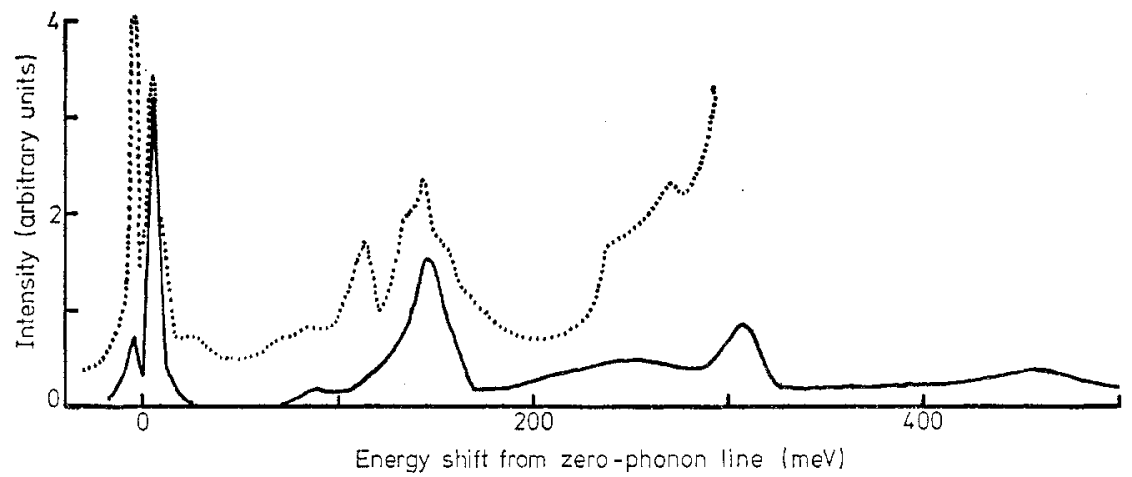

Figure 15. N9 luminescence at $100 \mathrm{~K}$ (full curve) and $80 \mathrm{~K}$ luminescence excitation (dots) spectra, plotted in terms of the energy shift from the zero-phonon doublet. The shift is negative for the lower spectrum (after Wight and Dean 1967, Dean and Male 1964a). The increase in luminescence excitation intensity at $300 \mathrm{meV}$ corresponds to the fundamental absorption edge.

excitation they are sharper and more easily studied (figure 15). They broaden relatively little at higher temperatures; the zero-phonon line is a doublet $(5.254$ and $5.263 \mathrm{eV}$ ), and the splitting is still resolvable at room temperature (Nahum and Halperin 1962). In luminescence the higher energy component of the doublet is very weak, indicating that the splitting occurs in the excited state. The thermal activation energy is $10 \mathrm{meV}$, consistent with the optical splitting (Wight and Dean 1967). The phonon sidebands in luminescence show less structure than in excitation, partially because one zero-phonon line is almost frozen out (figure 15). Some of the extra excitation peaks may be zero-phonon lines (Wight and Dean 1967); on the other hand, there may be a genuine departure from mirror symmetry. This could indicate a Jahn-Teller effect, though according to Davies (1974b) coupling is primarily to symmetric modes.

Structure beyond the fundamental absorption edge at $5.5 \mathrm{eV}$ is thought to be associated with N9. It is not detectable directly in absorption, but can be seen in luminescence excitation and in photoconductivity (Crowther and Dean 1967, Wight and Dean 1967, Denham et al 1967).

There is a weak band in excitation at $5.17 \mathrm{eV}(240 \mathrm{~nm})$, which Sobolev and Lisoivan (1972) claim correlates with the $5.26 \mathrm{eV}$ peaks, though Crowther and Dean (1967), denying this, label it N10. Another weak line at $5.28 \mathrm{eV}$ is visible in the excitation spectrum, though not in luminescence, and is probably due to a forbidden transition to a higher excited state. It is enhanced by uniaxial stress, consistent with this interpretation (Wight and Dean 1967, Crowther and Dean 1967). But Crowther and Dean were unable to determine the symmetry of N9 because of the closeness of the zero-phonon doublet and the inducing of the forbidden transitions. This is unfortunate, since the symmetry of the centre is an excellent guide to a model. The uniaxial stress results should be repeated in luminescence (not excitation) at liquid helium temperature; one member of the doublet would then be frozen out, as would transitions from the forbidden level, making the spectra easier to interpret; the $80 \mathrm{~K}$ spectra of Crowther and Dean would then be simpler to unravel.

There are two models for the N9 centre. The first, if one accepts the correlation with the B1 centre, is a dislocation loop on (111) planes which somehow involves nitrogen $-\mathrm{N}_{\mathrm{S}}$ (111) (Sobolev and Dubov 1975). (It is discussed in \$3.2.1.2). The 
second is a nearest-neighbour donor-acceptor pair in its neutral charge state (Dean $1965)(\$ 4.1 .3)$.

Since N9 is found principally in type Ia diamonds it probably involves a nitrogen aggregate, but further work is clearly necessary.

4.1.3. Band $A$ luminescence. A broadband luminescence between 1 and $3.7 \mathrm{eV}$ can be excited in virtually all diamonds by energetic particles ( $\alpha$, electrons) or photons (UV or $\mathrm{x}$-ray) (figure 17). It may display some structure due to superimposed luminescence or absorption of other systems such as N3, but is itself basically featureless. The bandwidth is typically $0.8 \mathrm{eV}$. The peak occurs at different energies for different types of diamond- $2 \cdot 2 \mathrm{eV}$ (type Ib synthetics), $2 \cdot 8 \mathrm{eV}$ (natural IIb), $3 \cdot 1 \mathrm{eV}$ (Ia and IIa) - and shifts to higher energy at high excitation density. Some crystals may show two peaks. The luminescence is prominent below room temperature, but is totally quenched at $400 \mathrm{~K}$, the activation energy for quenching being $0.36 \mathrm{eV}$. It is also quenched by radiation damage. Type IIa material may show weaker luminescence than type Ia (Dean and Male 1964b, Dean 1965, Crossfield 1974, Kiflawi and Lang 1974, Sobolev and Dubov 1975).

According to Dean (1965), band A luminescence is caused by recombination of electrons and holes at donor-acceptor pairs. This model predicts that the photon energy is given by:

$$
E=E_{\mathrm{g}}-\left(E_{\mathrm{A}}+E_{\mathrm{D}}\right)+\frac{e^{2}}{\epsilon r}-\frac{e a^{5}}{\epsilon r^{6}}
$$

where $E_{\mathrm{g}}$ is the indirect energy gap $(5.5 \mathrm{eV}), E_{\mathrm{A}}$ and $E_{\mathrm{D}}$ are the depths of the acceptor and donor states from the valence and conduction bands, $\epsilon$ is the dielectric constant and $r$ is the separation of the donor and acceptor. The final (polarisation) term, in which $a$ is an adjustable parameter, is negligible for large $r$. A consequence of this model is that the larger the separation $r$ between donor and acceptor, the lower the photon energy and the longer the decay time. Experimentally this does seem to be the case with band A luminescence (Dean 1965, Crossfield 1974, Sobolev and Dubov 1975). It seems that the $2.8 \mathrm{eV}$ peak is due to nearest-neighbour pairs, the $2.2 \mathrm{eV}$ peak to all the other, more distant, pairs. The supposition is that in natural crystals the donors and acceptors have drifted together, whereas in synthetics a more nearly statistical distribution obtains. Using an acceptor energy of $0.37 \mathrm{eV}(\$ 3.2 .3)$ and a donor energy of $4 \mathrm{eV}$ (Denham et al 1967) in the equation gives a threshold for luminescence of $1 \cdot 1 \mathrm{eV}$, as observed (Dean 1965, Crossfield 1974).

The model for the band A defect suggested by Sobolev and Dubov (1975) seems unable to account for the properties displayed. Dean's model accounts reasonably well for band $\mathrm{A}$ luminescence, though the acceptor is boron, but the $4 \mathrm{eV}$ donor seems unlikely to be the single nitrogen atom, since that lies $2 \mathrm{eV}$ deep (\$3.2.2). Further work is clearly necessary on this question and on the relationship, if any, between band $\mathrm{A}$ and $\mathrm{N} 9$.

4.1.4. The S1 centre $(503.4$ and $510.7 \mathrm{~nm}, 2.463$ and $2.429 \mathrm{eV})$. This centre occurs in the photoluminescence spectrum of some natural diamonds. At $85 \mathrm{~K}$ there are two zero-phonon lines at 2.463 and $2.429 \mathrm{eV}$; at $4 \mathrm{~K}$ the former freezes out and the latter is stronger (Sobolev et al 1969b, Nedzvetskii and Gaisin 1973a).

$\mathrm{S} 1$ is particularly interesting because the main zero-phonon line at $2.463 \mathrm{eV}$ almost coincides in energy with the $\mathrm{H} 3$ and $3 \mathrm{H}$ zero-phonon lines ( $\$ \$ 4.2$ and 4.3 ). 
But the three centres are quite distinct; their phonon replicas in luminescence are quite different, and only S1 shows the $2.429 \mathrm{eV}$ line. Furthermore, both S1 zero-phonon lines show a linear Stark effect (Kaplyanskii et al 1971), whereas for H3 it is quadratic.

H3 and S1 can coexist in the same crystal (Nedzvetskii and Gaisin 1973a). These authors also claim that $\mathrm{S} 1$ contains three other zero-phonon lines, at 2.393, 2.363 and $2.344 \mathrm{eV}$, but this has not been confirmed by other workers. Lines at similar energies are produced by irradiation (Burton 1972, Davies 1977a), so these three lines may be independent features.

According to Klyuev et al (1973), S1 tends to occur in crystals containing the single nitrogen centre, i.e. type $\mathrm{Ib}$ crystals. An EPR centre of $\mathrm{C}_{2 \mathrm{~h}}$ symmetry was discovered by Klingsporn et al (1970) in strongly luminescent type Ib diamonds. Shcherbakova et al (1972) found that all diamonds containing this EPR centre showed $\mathrm{S} 1$ luminescence, suggesting that they were the same defect. But the former shows a linear Stark effect, implying no inversion symmetry, whereas the EPR centre has a $\mathrm{C}_{2 \mathrm{~h}}$ point group, which contains the inversion operation.

Clearly, further work is necessary on the $\mathrm{S} 1$ centre, e.g. symmetry determination by polarised luminescence and uniaxial stress, measurement of thermal activation energy and determination of its absorption spectrum. The two zero-phonon lines should be correlated explicitly with each other and with the EPR centre, and their bleaching behaviour examined. Although $3 \mathrm{H}, \mathrm{H} 3$ and $\mathrm{S} 1$ are distinct centres, the similarity in energy of their zero-phonon lines may indicate a connection between them; this should be investigated.

4.1.5. Cathodoluminescence from dislocations. A blue luminescence, commencing at $2.4 \mathrm{eV}$ and extending to at least $3.1 \mathrm{eV}$, has been observed from dislocations in natural and synthetic diamonds. It is more than $90 \%$ polarised, the $E$ vector being parallel to the line of the dislocation, and is best observed in regions of the crystal where the blue band A luminescence is weak. Whether it is intrinsic to the dislocations or due to decoration by impurities is unclear. Disclocations can certainly be decorated by $\mathrm{H} 3$ defects, and hence emit the characteristic greenish luminescence. In synthetic crystals some dislocations emit in the yellow and red, though the luminescence bands have not been identified (Kiflawi and Lang 1974, 1976, Woods and Lang 1975, Hanley et al 1977).

4.1.6. The $1.40 \mathrm{eV}$ centre $(884 \mathrm{~nm})$. This defect is seen in cathodoluminescence, but not in photoluminescence or absorption, in natural and synthetic type Ib crystals (figure 16). The zero-phonon line at $1.40 \mathrm{eV}$, which contains one-fifth of the intensity of the system, is a doublet of $2.9 \mathrm{meV}$ separation. There is no change in relative intensity of the two components between 20 and $80 \mathrm{~K}$ and hence the splitting occurs in the ground state. In diamond the zero-phonon line energy usually decreases as $T$ increases (Davies 1972b), but in this case it increases. The defect's identity is unknown, but it probably involves the nitrogen donor (Dean 1965, Wight et al 1971).

4.1.7. Bands $B$ and $C$. Both these bands appear in cathodoluminescence (figure 17) but have not been recorded in absorption or photoluminescence. Band $B$ occurs in natural type II crystals, though it may be obscured in type I crystals by competing luminescence (Wight et al 1971).

Band C (which does not correlate with band B) is seen in all except type Ib. It is unusual in that the apparent zero-phonon line couples to a single very sharp 

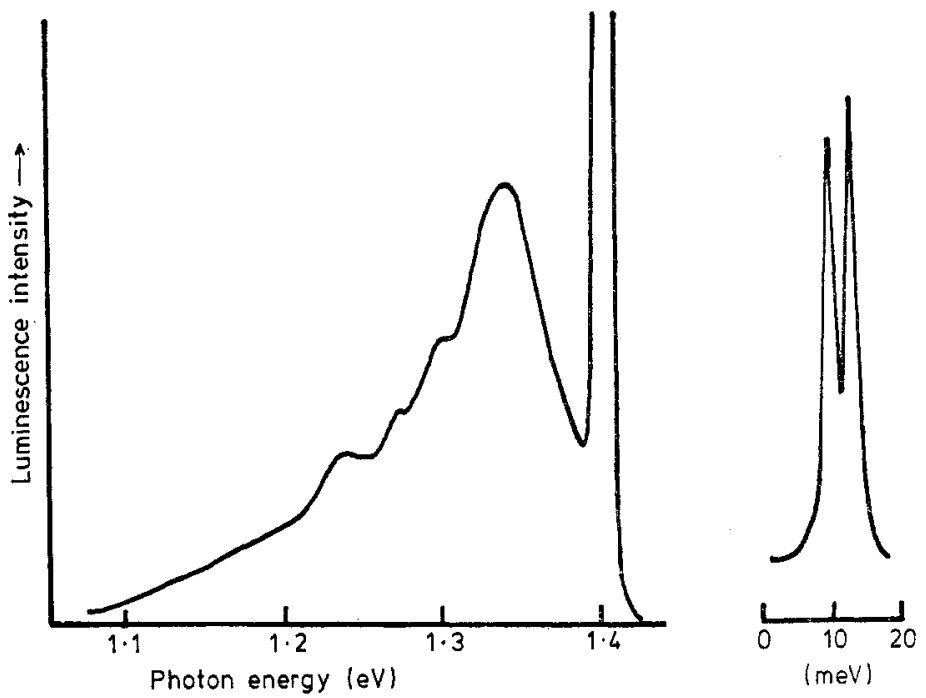

Figure 16. The $1.40 \mathrm{eV}$ centre cathodoluminescence at $80 \mathrm{~K}$. The $1.40 \mathrm{eV}$ zero-phonon line is a doublet, as shown on the right (after Wight 1968).

$53 \mathrm{meV}$ phonon, at least eleven replicas being resolved (Wight et al 1971). This phonon must be a resonant mode characteristic of the band $\mathrm{C}$ defect, implying either a defect of large mass or locally softened force constants.

4.1.8. The amber centre $(0.77 \mathrm{eV})$. This is common in, but not exclusive to, type Ib crystals. There is a zero-phonon line at $0.77 \mathrm{eV}$ with phonon replicas at 0.83 and $0.89 \mathrm{eV}$, and continuous absorption up to at least $3.5 \mathrm{eV}$ with structureless broad bands at 2.2 and $3.3 \mathrm{eV}$ (figure 18). In the infrared there is also a peak at $0.52 \mathrm{eV}$ and a characteristic single isotropic EPR line. The defect may involve nitrogen (du Preez 1965).

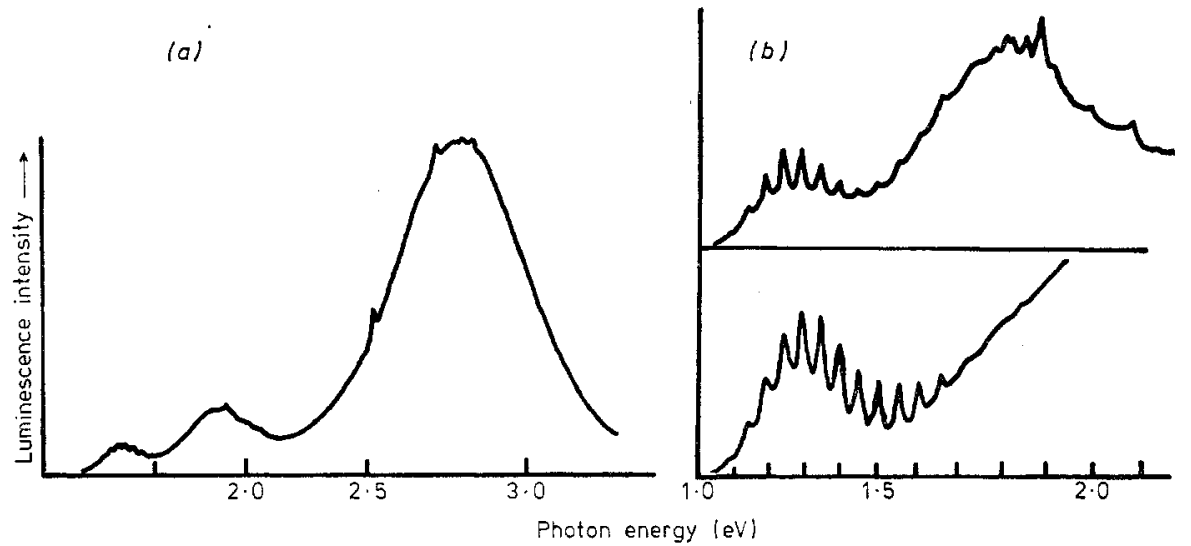

Figure 17. (a) The cathodoluminescence bands A, B and C. (b) Upper: same specimen as (a), but scale expanded. Lower: band $\mathrm{C}$ in a different crystal (after Wight 1968). 


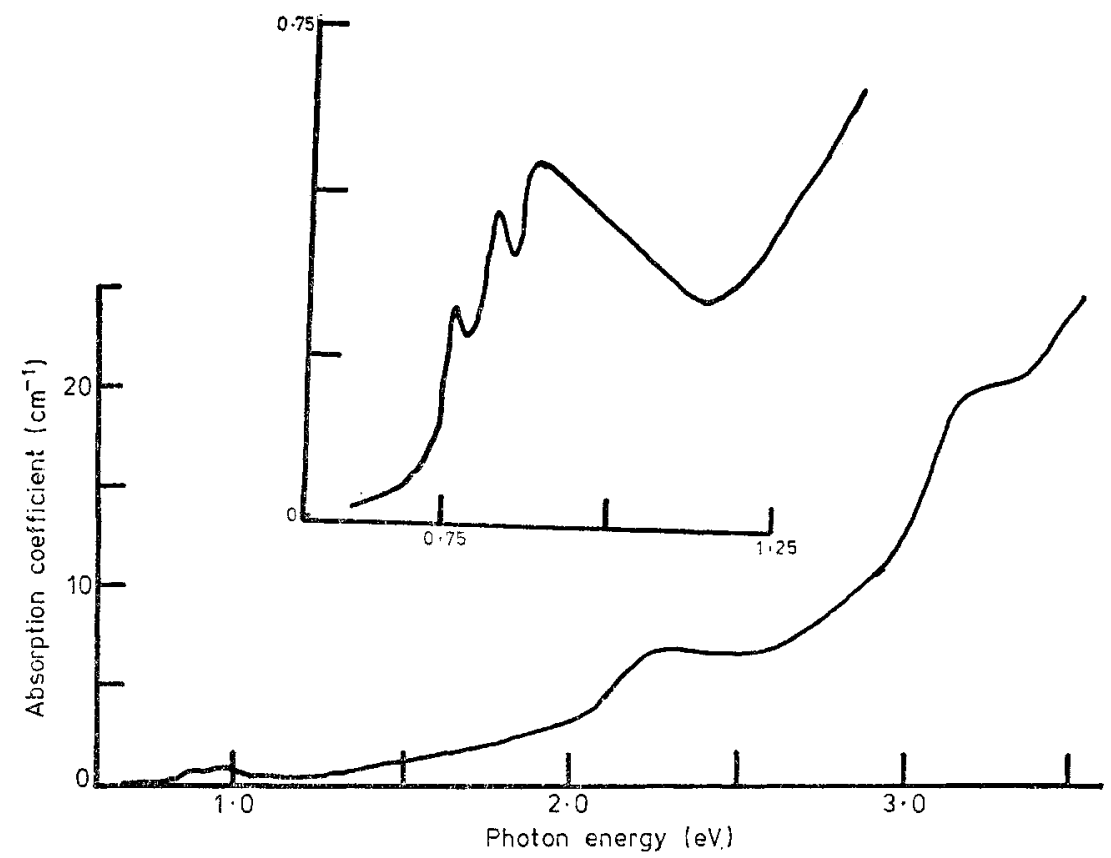

Figure 18. $80 \mathrm{~K}$ absorption spectra of the amber centre (after du Preez 1965). The scales are expanded in the inset.

\subsection{Irradiated diamond}

The wealth of spectra observed after room-temperature irradiation without annealing strongly suggests a mobile defect. The corollary is that any radiation defects produced without a high-temperature anneal must be either intrinsic or must involve a trapped interstitial, since the vacancy (the GR1 centre) is immobile below $900 \mathrm{~K}$.

4.2.1. The $G R 1$ centre $(741 \mathrm{~nm}, 1 \cdot 673 \mathrm{eV})$. There are a pair of zero-phonon lines at 1.665 and $1.673 \mathrm{eV}(744.5$ and $741.0 \mathrm{~nm})$, a broad vibronic band peaking at $2 \mathrm{eV}$ ('the GR1 band') and further zero-phonon lines between 2.8 and $3.1 \mathrm{eV}$ (GR2, GR3, .., GR8) (figures 19 and 20) superimposed on a broad band ('the Uv band') peaking at $4 \mathrm{eV}$. It is now generally accepted that the GR1 defect is the neutral vacancy. It is interesting and important for the anomalous effects it shows, and for its relevance to theoretical solid-state physics. Its properties have previously been reviewed by Clark and Mitchell (1971a, b, 1976), Clark et al (1979) and Davies (1977a).

Irradiation of any diamond produces a blue-green coloration due to the absorption bands in the red and violet (figure 19). The strength of the GR1 band is linearly related to the irradiation dose (Clark et al 1956a, 1961). This indicates an intrinsic lattice defect-no impurity is involved.

Irradiation of type IIb diamonds leads to the disappearance of the acceptor peaks in the infrared, and the early work indicated that the GR1 absorption band did not appear until all the acceptors had been compensated. Hence it is usually assumed that the GR1 centre is a donor and compensates the acceptors (Wedepohl 1957b, Clark et al 1961, Dyer and Ferdinando 1966; see also §3.2.3). (This incidentally 


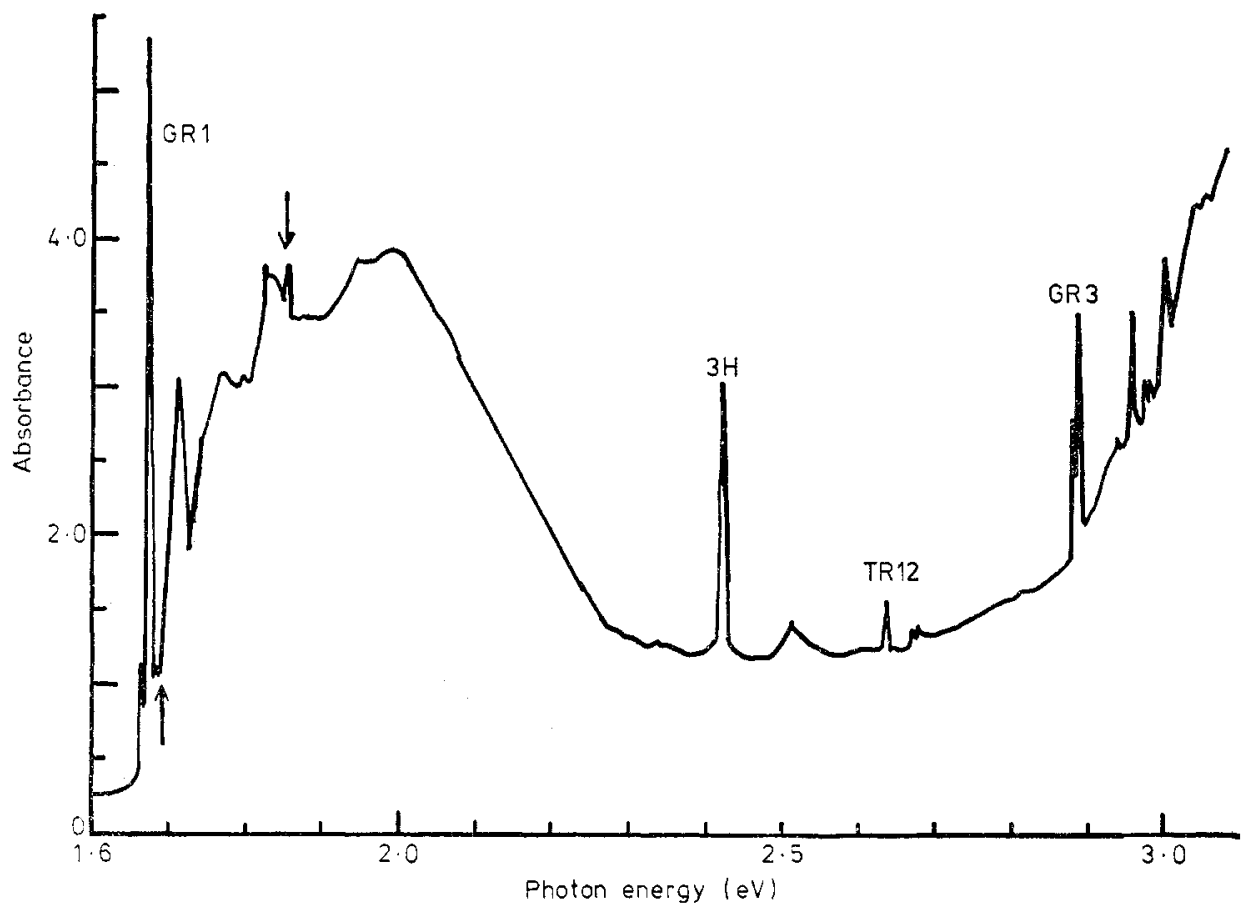

Figure 19. $80 \mathrm{~K}$ absorption spectrum of an electron-irradiated type IIb diamond. The two arrowed peaks belong to the tetragonal defect. The 3H and TR12 spectra are indicated; otherwise, all the structure is due to the GR defect. Before irradiation there was negligible absorption in this region.

implies that positively charged and hence paramagnetic vacancies are present in such crystals, though they have apparently not yet been observed.) However, Collins (1977) has detected the GR1 zero-phonon line in diamonds in which not all the acceptors have been compensated, and the GR1 centre annealed out below $1100 \mathrm{~K}$, whereas the acceptor spectrum re-appeared only above $1200 \mathrm{~K}$ in the same crystal. Furthermore there is evidence that the GR1 centre ionises by emitting a hole (Vermeulen et al 1974), i.e. acceptor-like behaviour-this is discussed in more detail below. Hence the picture is somewhat ambiguous at present; it may be that the defect can be either a donor or an acceptor, depending on the Fermi level, like the vacancy in silicon (Watkins 1965).

Clark et al (1961) deduced for diamond a displacement energy (i.e. the energy needed to displace a lattice atom from its site) of $80 \mathrm{eV}$. They used both the production of the GR1 band at room temperature in type IIa diamond and the carrier removal rate at $120 \mathrm{~K}$ in natural semiconducting diamond (though the samples were warmed to room temperature after irradiation, and conductivity measurements were made between 200 and $300 \mathrm{~K}$ ). Bourgoin and Massarani (1976), using synthetic boron-doped crystals, irradiating at $15 \mathrm{~K}$ and measuring the conductivity at $12 \mathrm{~K}$, deduced a displacement energy of $35 \mathrm{eV}$, which they attributed to the formation of vacancy-interstitial pairs. There is not necessarily a conflict if we assume that the result of Clark et al applies to isolated vacancies, particularly as we know that there are annealing stages below room temperature (Lomer and Wild 1971, Massarani and Bourgoin 1976) and that temperature affects the damage rate (Collins 1977), but further work is desirable to explain the discrepancy. 


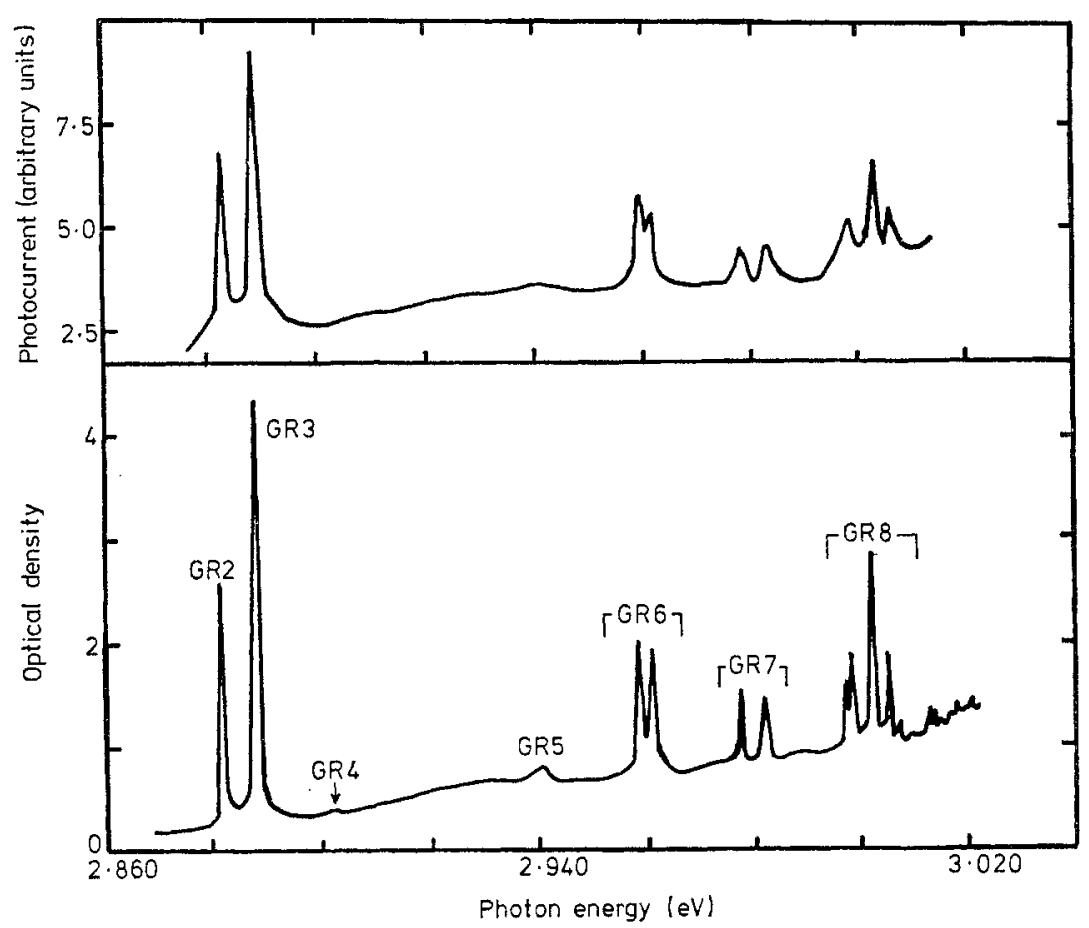

Figure 20. Photoconductivity (upper) and absorption spectrum (lower) at $40 \mathrm{~K}$ of the GR2GR8 lines. Instrumental resolution is lower in the photoconductivity spectrum (after Vermeulen et al 1974). Compare with figure 19.

The GR1 absorption is strong; the oscillator strength is about $0 \cdot 1$. The luminescence is weak, but is an approximate mirror image of the absorption, and is unpolarised-an important result. The luminescence excitation spectrum shows peaks corresponding to GR3 and GR6, with a broad band at higher energies--the first evidence of a relation between these transitions and GR1 (Clark and Norris 1971).

It has been suggested (see Clark and Mitchell 1971) that the broad peak in the GR1 absorption spectrum at $1.71 \mathrm{eV}$ is a zero-phonon line, but this now seems unlikely. A corresponding peak appears in luminescence at $1.63 \mathrm{eV}$ indicating that it is a phonon replica of the main zero-phonon line at $1.673 \mathrm{eV}$. Such a low phonon energy may be due to local softening of the crystal round the defect (Ritter 1970, Larkins and Stoneham 1971). Accurate measurements (Wight et al 1971, Nedzvetskii and Gaisin 1973b) find that this first phonon energy is $41 \mathrm{meV}$ in absorption but $36 \mathrm{meV}$ in luminescence. Such a discrepancy also occurs with the H3 and H4 defects, consistent with them being due to the vacancy trapped at an impurity.

The $1.673 \mathrm{eV}$ zero-phonon line has a weak satellite at $1.665 \mathrm{eV}$ which is visible at $80 \mathrm{~K}$ but freezes out at $4 \mathrm{~K}$ (Lannoo and Stoneham 1968, Clark and Walker 1972, 1973). Both lines have been studied under uniaxial stress, and the energy level diagram derived from this and from thermal studies is shown in figure 21 (Clark and Walker 1973, Davies and Penchina 1974, Davies 1974a, 1975a). The defect has the full $T_{d}$ symmetry of the diamond point group.

A tetrahedral defect would have unpolarised luminescence (table 4), as Clark and Norris (1971) found for the GR1 luminescence band as a whole, and B J Parsons 

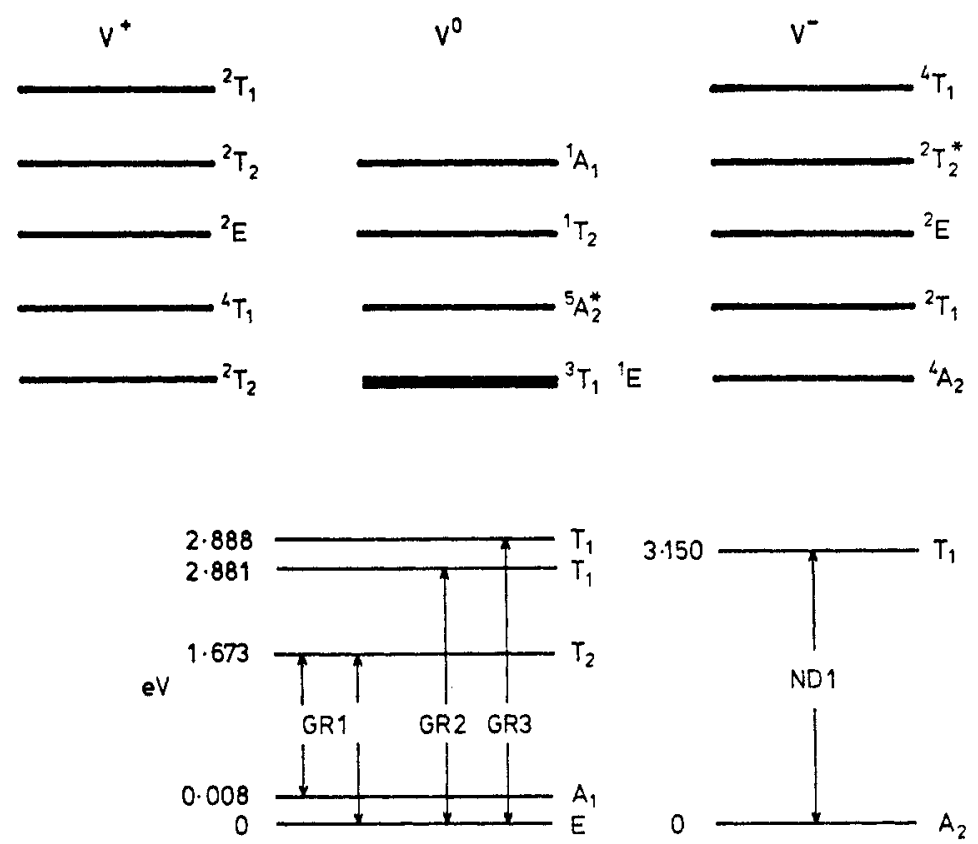

Figure 21. Upper: the energy levels of the positive, neutral and negative vacancies in diamond according to the 'defect molecule' calculations of Coulson and Larkins (1971). For some values of the parameters used in the calculations the asterisked levels may be the ground state. Lower: the experimentally determined energy levels for the GR and ND1 defects.

(1972 personal communication) confirmed for the zero-phonon lines at 1.665 and $1.673 \mathrm{eV}$. The $1.673 \mathrm{eV}$ line shows a linear Stark shift (Kaplyanskii et al 1971), consistent with tetrahedral symmetry. The GR1 ground state is known to be diamagnetic (Clark et al 1964). Magnetic circular dichroism measurements (Douglas and Runciman 1977a) indicate a degenerate excited state and confirm the diamagnetic ground state, consistent with the $\mathrm{T}$ and $\mathrm{E}$ assignments already discussed.

Thus, all the experiments agree in assigning the GR1 energy levels as a ${ }^{1} \mathrm{E}$ ground state, with a ${ }^{1} \mathrm{~A}$ just above and $\mathrm{a}^{1} \mathrm{~T}$ at $1.673 \mathrm{eV}$ (figure 21). 'The experiments cannot distinguish between $A_{1}$ and $A_{2}$, and $T_{1}$ and $T_{2}$, but since these results are very similar to the ones predicted for $\mathrm{V}^{0}$ by the molecular orbital calculations (Coulson and Larkins 1971, Stoneham 1975) $A_{1}$ and $T_{2}$ are usually chosen $\left(A_{1}-T_{1}\right.$ is forbidden). Hence these recent experiments confirm the long-standing belief that the GR1 defect is the neutral vacancy. Furthermore they indicate that the 'defect molecule' approach is a reasonable way of calculating the energy levels of point defects in semiconductors-a very important result.

Since the GR1 ground state is degenerate, a Jahn-Teller effect is expected; the fact that the Huang-Rhys factors derived from the zero-phonon line intensity, the Stokes shift and the bandwidth ( $\$ 2.1)$ are not equal $(3 \cdot 7,5 \cdot 7$ and $9 \cdot 2$, respectively) supports that expectation (Stoneham 1975). It appears that the $E$ and $A$ states (figure 21) are vibronic states derived from an $\mathrm{E}$ electronic state coupled to lattice vibrations of e symmetry. The $\mathrm{T}_{2}$ state also couples to e modes. The Jahn-Teller energy is $0.63 \mathrm{eV}$ and the phonon energy is $130 \mathrm{meV}$. Another level is predicted $20 \mathrm{meV}$ above the ground state, but it has not been observed (Lowther 1975, 1976, 
Stoneham 1977a). An earlier suggestion (Clark and Walker 1973), that the A state derived from the ${ }^{3} \mathrm{~T}_{1}$ state (figure 21 ) as a result of spin-orbit splitting, now seems unlikely (Davies 1974a, Lowther 1975, 1976, Stoneham 1977a). The low $g$ value in the $\mathrm{T}$ state (Douglas and Runciman 1977a) is a result of Jahn-Teller quenching (Lowther and Stoneham 1978).

The GR1 absorption and luminescence now appear to be understood. This is not yet the case for the GR2-GR8 transitions (figures 19,20 and 21). These lines, although recorded by Clark et al (1956a) and other authors, received little attention until recently. We now know that they are all transitions at the GR1 defect (because they correlate in intensity with the GR1 zero-phonon line, show similar photoconductivity and thermal behaviour, and the most prominent of them appear in the GR1 excitation spectrum). There are more than seven lines; GR6 and GR7 are doublets and GR8 a quintet (figure 20). GR2 and GR3 have been studied under uniaxial stress and confirmed as E-T transitions at the GR1 defect; the upper state is $T_{1}$ if the GR1 upper state is $T_{2}$ (and vice versa), which explains why they, unlike the $1.673 \mathrm{eV}$ line, do not have a satellite-it is forbidden (Clark and Norris 1971, Clark and Walker 1972, Walker et al 1974, Davies and Foy 1978). Douglas and Runciman (1977b) have suggested that GR5 and GR8 may not be transitions at the GR1 centre, but the differences they found between a Ia and a IIa crystal are probably due simply to large linewidths in the former.

Farrer and Vermeulen (1972) studied the photoconduction behaviour of all the GR lines and found photoconductivity troughs corresponding to the GR1 absorption peaks, as expected-photons absorbed by the localised levels of the GR1 centre are not available for photoconductivity (cf process 2 of figure 12). However, there were sharp photoconductivity peaks corresponding to the GR2-GR8 absorption peaks (figure 20)-a totally unexpected and currently not completely explained phenomenon. (The ND1 centre shows similar behaviour.) When photoconduction peaks are observed they are usually broad ( $1 \mathrm{eV}$ or more), corresponding to quasi-localised levels within a conduction or valence band. Photothermal ionisation (process 4 of figure 12) cannot be the explanation because the GR2 excited state must be at least $120 \mathrm{meV}$ from the conduction band (i.e. $120 \mathrm{meV}$ below GR8), but photoconductivity is observed at $40 \mathrm{~K}$ (where $k T=3.4 \mathrm{meV}$ ); and furthermore the relative intensities of the lines are the same in photoconductivity and absorption whatever the temperature, which would not be the case in photothermal ionisation (compare figures 10 and 20).

Another puzzling feature is that the GR centre has been regarded as a donor, as we have seen, since irradiation leads to compensation of the acceptor centres. Yet the evidence is that the GR2-8 photocurrent is due to holes (Vermeulen et al 1974, Vermeulen 1976). It seems unlikely that the GR2-8 excited levels can be within the conduction or valence bands, and autoionisation is probably involved. Three mechanisms have been proposed. Stoneham (1977b) has suggested that GR2 is a twoelectron transition; it de-excites by autoionisation in which an electron is lifted out of the valence band. He further suggests that GR3-GR8 correspond to vibronic structure on the GR2 transition. This seems unlikely since they are so sharp, but detailed calculations using this model are desirable.

Dunn (1976), using the energy levels calculated for $\mathrm{V}^{0}, \mathrm{~V}^{+}$and $\mathrm{V}^{-}$by Coulson and Larkins (1971) (figure 21) but taking ${ }^{3} \mathrm{~T}_{1}$ as the ground state of $\mathrm{V}^{0}$, has proposed that the GR lines are bound-exciton transitions from the ground state of the neutral vacancy to the ground state (GR1) or excited states (GR2-8) of a $\mathrm{V}^{-}$which has trapped a hole. These discrete transitions overlap with continua due to emission 
of holes from $\mathrm{V}^{+}$and $\mathrm{V}^{0}$, and hence autoionisation can occur. However, the GR1 ground state is not paramagnetic, as demanded by this model, and it is not clear whether the calculations will work with a ${ }^{1} \mathrm{E}$ ground state for $\mathrm{V}^{\mathrm{D}}$.

Lowther (1977) has proposed a similar model to Dunn, involving transitions between a neutral vacancy and a hole combined with a negative vacancy. He points out that GR2-GR8 are very sharp, which suggests that the hole is located in one of the basal orbitals of the nearest-neighbour carbon atoms surrounding the vacancy (i.e. the orbitals connecting these carbon atoms to the next-nearest neighbours). These orbitals lie very close to or just within the valence band, permitting photoconductivity while allowing the absorption lines to remain sharp. The hole de-excites into the ${ }^{4} \mathrm{~A}_{2}$ ground state (figure 21) of $\mathrm{V}$-, and luminescence from GR2-8 should be significantly different to that from GR1. Although the model is not without its difficulties, it is the most detailed and the most promising one to date. However, the luminescence prediction is difficult to test. Although Clark and Norris (1971) have shown that GR1 luminescence can be excited by photon energies corresponding to GR2-8, the author was unable to detect any GR2-8 luminescence (nor any luminescence corresponding to transitions between the GR1 and GR2-8 excited states, despite the fact that $T_{1}-T_{2}$ transitions are allowed-see figure 21 ).

Dyer and du Preez (1965) proposed that the uv band was due to transitions from the GR1 centre into the conduction band. However, this would lead to problems in explaining the sharpness of GR2-8, as we have seen. A more likely explanation is that part, if not all, of it is the vibronic sideband of the GR2-8 zero-phonon lines (Davies 1975c).

As Davies (1977a, b) has emphasised, there is much we still do not know about the GR centre. Further work is desirable, particularly on its possible connection with ND1 (see §4.2.2).

4.2.2. The ND1 centre $(394 \mathrm{~nm}, 3 \cdot 15 \mathrm{eV})$. The defect can be created in all types of diamond, though in IIa and IIb it is normally weak. In Ib it is created during an $80 \mathrm{~K}$ irradiation; in Ia, anneals of $620 \mathrm{~K}$ are necessary after irradiation and its strength qualitatively correlates with the nitrogen concentration. Coupling is predominantly to a 'breathing-mode' phonon of $80 \mathrm{meV}$ (Clark et al 1956a, Dyer and du Preez 1965, 1967, Davies and Lightowlers 1970, Davies 1974b).

Dyer and du Preez $(1965,1967)$ and Davies (1977a) have investigated in detail the photochromic and thermochromic behaviour of ND1. Heating at $770 \mathrm{~K}$ enhances the intensity of ND1 and of a single isotropic EPR line which is induced by irradiation, whilst GR1 and the Uv band are diminished. Ultraviolet light of energy less than $3.15 \mathrm{eV}$ has the same effect, but if the energy is greater than $3.15 \mathrm{eV}$ (i.e. within the ND1 absorption system) it is ND1 that is bleached and GR1 enhanced (figure 22). Annealing above $800 \mathrm{~K}$ destroys ND1.

The ND1 defect has tetrahedral symmetry, the ground state being $A$ and the excited state T (Runciman 1965, Davies and Lightowlers 1970, Kaplyanskii et al 1971, Douglas and Runciman 1977b). The centre is not luminescent (Davies and Lightowlers 1970, Davies 1972b), contrary to earlier reports. This is presumably because, like GR2-8, there are photoconductivity peaks corresponding to the absorption peaks; these are present even at $8 \mathrm{~K}$, which rules out photothermal ionisation, and they are still unexplained (Farrer and Vermeulen 1972).

A tetrahedral defect must be either a single vacancy, a single interstitial or a substitutional impurity. Davies and Lightowlers (1970) proposed a nitrogen interstitial, 


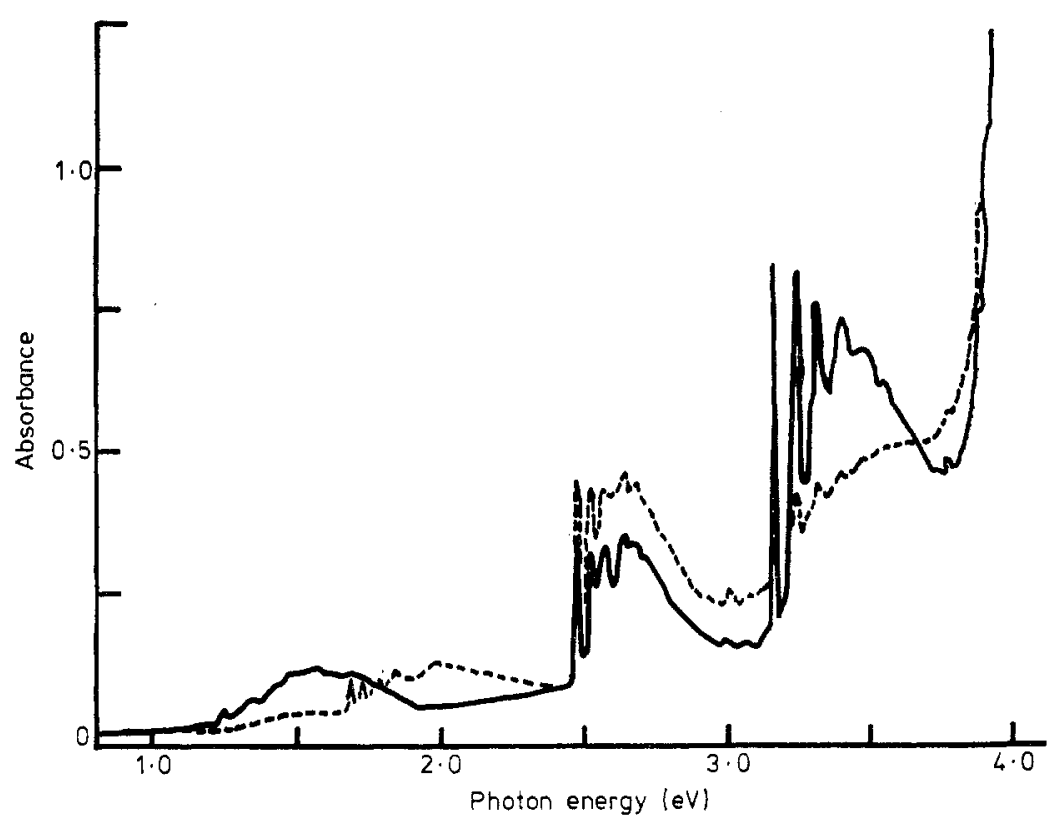

Figure 22. $80 \mathrm{~K}$ absorption spectrum of an irradiated type Ia diamond. Full curve: after heating in the dark; broken curve: after subsequent exposure to unfiltered mercury light. The GR1 $(1.673 \mathrm{eV})$ and ND1 $(3.15 \mathrm{eV})$ bands vary in a complementary fashion, as do $\mathrm{H} 2(1.25 \mathrm{eV})$ and $\mathrm{H} 3(2.46 \mathrm{eV})$ (after du Preez 1965).

since the centre is created in strength only in nitrogen-containing diamonds. But Sobolev and Eliseev (1976) and Davies (1977b) have recently suggested that ND1 is a negative vacancy. Davies' arguments are as follows. Firstly, GR1 is very weak in type I compared to type II diamonds, the ratio GR1/ND1 being $18,1.3$ and $\ll 0.1$ in a IIb, Ia and Ib irradiated under similar conditions. Since GR1 is a very stable defect this suggests that vacancies are being created in a different charge state. Secondly, the molecular orbital calculations (see figure 21) find that $\mathrm{V}$ - has a ${ }^{4} \mathrm{~A}_{2}$ ground state with a ${ }^{4} \mathrm{~T}_{1}$ state several $\mathrm{eV}$ above, similar to ND1. Thirdly, the photochromic effects are more easily explained as a single defect changing its charge state, and the carrier emitted when the GR defect ionises does appear to be a hole (Vermeulen et al 1974), consistent with a change from $\mathrm{V}^{0}$ to $\mathrm{V}^{-}$. The carrier released when ND1 ionises has not been determined explicitly, but Vermeulen's (1976) work is consistent with electrons. Fourthly, the $\mathrm{H} 3$ centre is thought to contain a vacancy, and its growth on annealing may match the decrease in either GR1 or ND1. Fifthly, a ${ }^{4} \mathrm{~A}_{2}$ ground state, with spin 3/2, should be EPR-active; Dyer and du Preez (1965) have indeed found an EPR signal correlating with the ND1 absorption, though it has not been analysed in detail. Finally, Lowther and Stoneham (1978) have shown that the ND1 uniaxial stress parameters are consistent with a negative vacancy. Hence, Davies' hypothesis seems to fit the facts, and if correct it is a very important corroboration of the Coulson molecular orbital calculations (Coulson and Larkins 1971). Further tests of the hypothesis, by determining the sign of the ND1 charge carriers, and particularly by EPR studies, are desirable.

4.2.3. The $3 \mathrm{H}$ centre $(503.5 \mathrm{~nm}, 2.462 \mathrm{eV})$. This centre is produced in all types of diamond by irradiation, even at $80 \mathrm{~K}$, though more readily at room temperature. 
Its intensity is enhanced by short anneals at $600 \mathrm{~K}$, but longer times or higher temperatures cause it to disappear, as does UV irradiation. This is a charge transfer effect rather than a genuine anneal, because the centre can be restored by $x$-irradiation, and is stable to at least $900 \mathrm{~K}$. In type Ia its strength is proportional to the nitrogen content, and it is particularly strong in type Ib crystals. Whether this is a Fermi level effect or implies that nitrogen is involved in the centre is not clear. Phonon coupling is weak $(S \sim 0 \cdot 7)$, the major phonon replica being at $67 \mathrm{meV}$ (figure 23). Uniaxial stress indicates a $\pi\langle 110\rangle$ oscillator at a rhombic I centre (table 4) (Palmer 1961, Davies 1974a, Walker 1974, 1976a, 1977b, Nedzvetskii and Gaisin 1975).

The centre was labelled $3 \mathrm{H}$ because its zero-phonon line is at virtually the same energy as $\mathrm{H} 3$. (In earlier work, $3 \mathrm{H}$ was probably assumed to be $\mathrm{H} 3$.) Both centres have the same rhombic I symmetry (Walker 1974, Davies et al 1976). However,

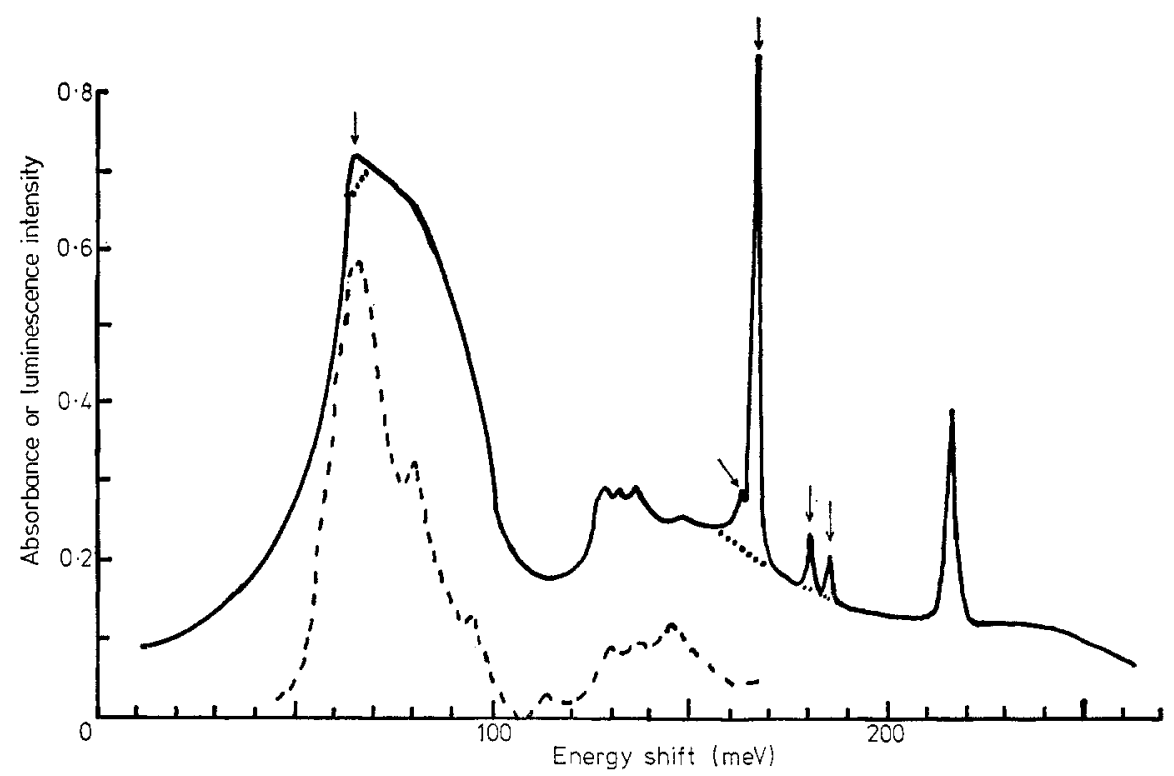

Figure 23. A comparison of the $3 \mathrm{H}$ absorption (broken curve) and photoluminescence (full curve) at $80 \mathrm{~K}$. The abscissa shows shift in energy from the zero-phonon line at $2.462 \mathrm{eV}$ and is negative for the luminescence spectrum. The arrowed peaks may be due to the laser used to excite the luminescence (after Walker 1977b).

there is no evidence of any relationship between them; $\mathrm{H} 3$ can be produced only in type Ia diamonds, whereas $3 \mathrm{H}$ can occur in considerable strength in all types, and their luminescence spectra are quite different (figures 23 and 28) (Walker 1976a, 1977b). For similar reasons $3 \mathrm{H}$ is presumably not the same defect as $\mathrm{S} 1$, which also has a $503.5 \mathrm{~nm}$ zero-phonon line ( $\$ 4.1 .4)$.

Hanley et al (1977) have observed $3 \mathrm{H}$ in cathodoluminescence in the surface layers of natural diamonds, presumably a result of natural irradiation. They report that on one occasion, using an intense electron beam, the $3 \mathrm{H}$ luminescence faded, to be replaced by the $575.5 \mathrm{~nm}$ system $(\$ 4.3)$. Since this latter can also be produced in any diamond by irradiation, a connection between them seems possible.

There is only one, speculative, model for $3 \mathrm{H}$, an oxygen-vacancy pair (Walker 1976a). Because $3 \mathrm{H}$ can be bleached, it must be paramagnetic in at least one charge state, and this should be investigated. Further studies of its luminescence spectrum 
and Stark shift are desirable, to confirm beyond doubt that it is not the same centre as $\mathrm{S} 1$.

4.2.4. The S2 centre. This centre was observed in the $80 \mathrm{~K}$ photoluminescence spectra of a few natural crystals. It has five zero-phonon lines A-E at 2.371, 2.537, $2.597,2.626$ and $2.637 \mathrm{eV}$, the three strongest of which $(\mathrm{A}, \mathrm{B}$ and $\mathrm{C})$ display phonon replicas. Going from $80 \mathrm{~K}$ to $140 \mathrm{~K}$ causes B to weaken dramatically, while $\mathrm{C}$ and D increase in intensity and $\mathrm{A}$ and $\mathrm{E}$ stay approximately constant (Il'in et al 1971). These changes are presumably related to population and depopulation of electronic levels.

The S2 system has also been recorded in absorption ( $\mathrm{J}$ Walker, unpublished work) in Ia diamonds, though not in IIa or IIb, after room-temperature electron irradiation. The strength was specimen-dependent. Only $A$ and $B$ were strong enough to measure, though weak peaks were present at energies corresponding to $\mathrm{C}, \mathrm{D}$ and $\mathrm{E}$. There were phonon replicas of $\mathrm{A}$ at 32 and $72 \mathrm{meV}$ (in good agreement with Il'in et al), each of intensity one-quarter that of A, giving a Huang-Rhys factor of 0.4 . A was clearly observable at $80 \mathrm{~K}$ but decreased in intensity at lower temperature, indicating that it is a transition from an excited state about $10 \mathrm{meV}$ above the ground state. $\mathrm{B}$ was $2 \cdot 5$ times as strong as $\mathrm{A}$, but less sharp (16 meV compared to $4 \mathrm{meV}$ ). Its intensity did not vary with temperature, and at $17 \mathrm{~K}$ it looked like an unresolved triplet or quartet. Further irradiation caused S2 to decrease in intensity, as did annealing at $600 \mathrm{~K}$; whether it could be restored by illumination was not investigated.

Other authors (Clark et al 1956a, Dean and Male 1964b, Wight 1968, Burton 1972, Davies 1974a, Davies and Hamer 1976) have recorded zero-phonon lines very close in energy to all of the S2 transitions, particularly in irradiated type Ib crystals. Further work is clearly necessary, firstly to confirm that the five lines A-E genuinely belong to the same centre, secondly to ascertain their relationship to the other work and, thirdly, to investigate the suggestion of Klyuev et al $(1972,1973)$ that $\mathrm{S} 2$ is related to the $\mathrm{N} 9$ and $\mathrm{B} 1$ centres.

4.2.5. The TR defect. This defect was first studied by Clark et al (1956a, b), who observed it in absorption and luminescence in irradiated type IIa crystals (figure 19). TR12, TR12a and TR17 $(2.638,2.645$ and $2.828 \mathrm{eV})$ are zero-phonon lines; the others, including TR13-TR16 (figure 24), though relatively well resolved, are probably phonon replicas of TR12. The Huang-Rhys factor is $0 \cdot 8$. There are also several infrared peaks between 1.52 and $1.54 \mathrm{eV}$ which appear to correlate qualitatively with TR12. Short anneals at $800 \mathrm{~K}$ enhance the system; longer times or higher temperatures destroy it. The luminescence spectrum is not a mirror image of the absorption, which suggests a Jahn-Teller effect. Uniaxial stress identifies TR12 as a $\sigma$ oscillator at a monoclinic I defect (table 4) (Walker 1974, 1977b).

The TR absorption is strengthened by short $800 \mathrm{~K}$ anneals but longer times or higher temperatures destroy it. It occurs in type IIa and IIb crystals, but not in type Ia (Walker 1977b). Nor was it detected in absorption or cathodoluminescence in irradiated synthetic type Ib diamonds (Burton 1972), though it was plainly visible in both absorption (Walker 1977b) and cathodoluminescence (Collins 1977) after lower doses in type IIb. G Davies (1976, personal communication) has observed a weak TR12 line in a type Ia crystal, but this may have been due to specimen inhomogeneity. 


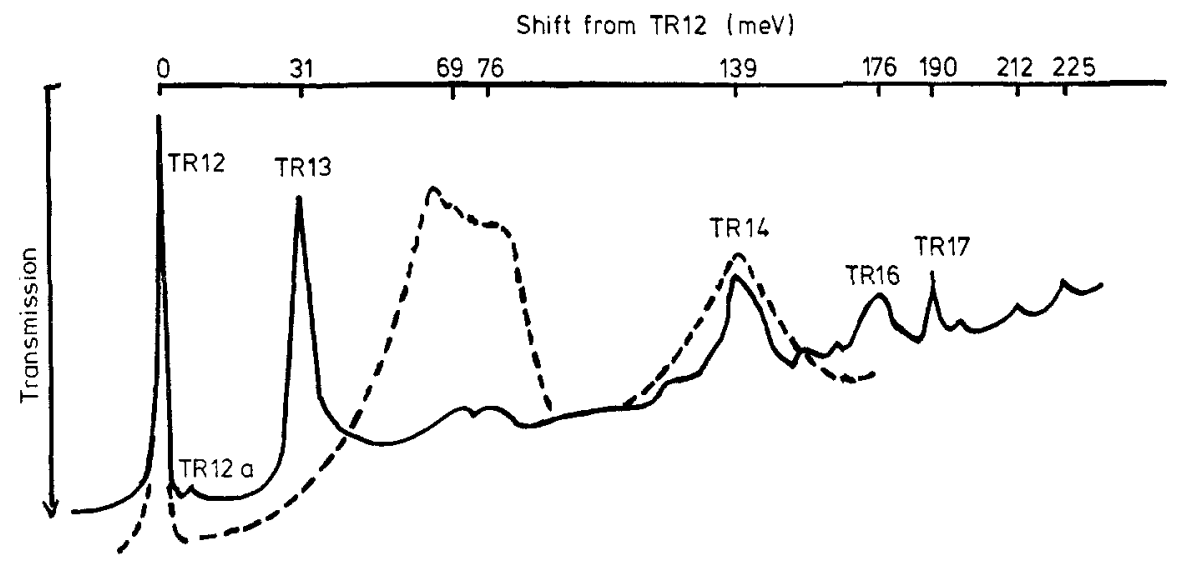

Figure 24. A comparison of the TR absorption (full curve) and photoluminescence (broken curve). The abscissa shows shift in energy of the various peaks from the TR12 zero-phonon line at $2.638 \mathrm{eV}$ and is negative for the luminescence spectrum. Note the absence of 'mirror symmetry' (after Walker 1977b).

The defect is thought to be an interstitial complex, possibly involving an impurity (Walker 1977b). The failure to produce it in type I crystals was ascribed to the presence of alternative traps for the carbon interstitials, but in view of Davies' (1977b) results for GR1 and ND1 it may be that the TR defect is simply in a different charge state in such diamonds.

4.2.6. The $5 R L$ defect $(270.6 \mathrm{~nm}, 4.581 \mathrm{eV})$. This unusual system (figure 25) was discovered in cathodoluminescence by Wight (1968). The main zero-phonon line $\mathrm{C}$ at $4.581 \mathrm{eV}$, together with its phonon structure down to $4.4 \mathrm{eV}$ and two other zerophonon lines $\mathrm{H}$ and $\mathrm{I}$ at $4.407 \mathrm{eV}$ and $4.389 \mathrm{eV}$, are reproduced very sharply at intervals of $236 \mathrm{meV}$. The defect has been observed after electron or neutron irradiation in 'intermediates' and types IIa and IIb (natural and synthetic), but not in types Ia or Ib, possibly because of competing absorption. Wight suggests that the

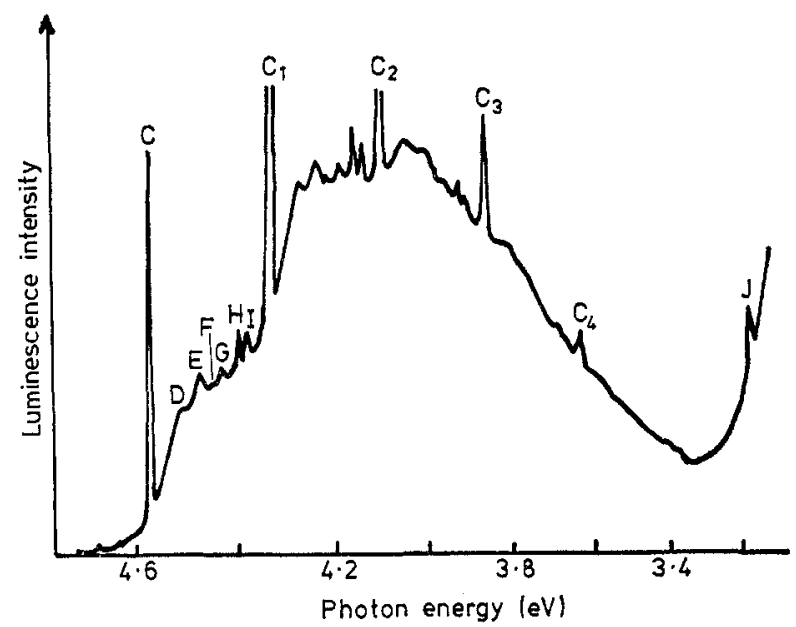

Figure 25. $100 \mathrm{~K}$ cathodoluminescence spectrum of the $5 \mathrm{RL}$ system in a type IIb diamond (after Wight 1968). 
defect is intrinsic, though perhaps an aggregate. Collins $(1977,1978)$ has observed that the centre anneals below $1200 \mathrm{~K}$, and has recorded the zero-phonon line in absorption.

The phonon energy of $236 \mathrm{meV}$ is well outside the Raman energy of $165 \mathrm{meV}$. It must be a local mode phonon, though there is no record of a peak at $236 \mathrm{meV}$ in the infrared after irradiation (Smith 1961). Further work on the 5RL centre is highly desirable.

4.2.7. The $2 B D$ defect. This defect was discovered by Wight (1968) in cathodoluminescence (figure 26) in type IIb diamonds containing the 5RL system, and hence is thought to be due to irradiation ('2BD' stands for 'type IIb damage'). There appear to be two zero-phonon lines, F and G. F consists of four components, the strongest being at $4.777 \mathrm{eV}$, with weaker peaks at $4.781,4.803$ and $4.830 \mathrm{eV}$; they couple to a sharp $210 \mathrm{meV}$ phonon. $\mathrm{G}(4 \cdot 698 \mathrm{eV})$ is a similar multiplet, the phonon

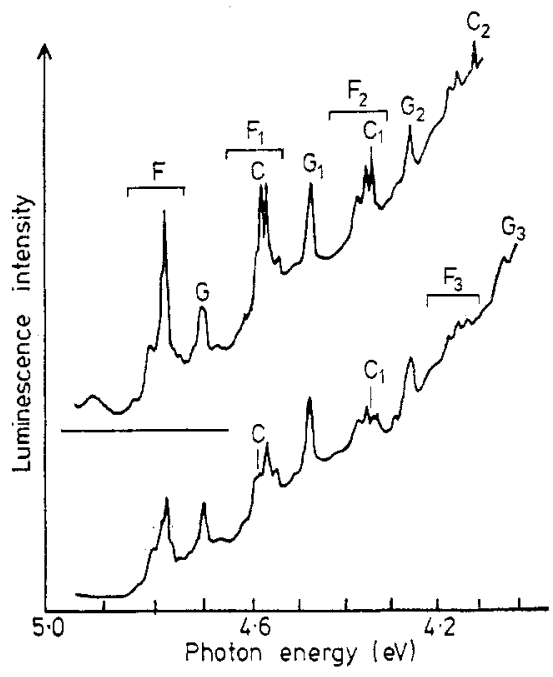

Figure 26. The $2 \mathrm{BD}$ cathodoluminescence spectrum in two diamonds, at $50 \mathrm{~K}$ (upper) and $100 \mathrm{~K}$ (lower). Peaks $\mathrm{C}, \mathrm{C}_{1}$ and $\mathrm{C}_{2}$ are part of the $5 \mathrm{RL}$ system (see figure 25) (after Wight 1968).

energy in this case being $220 \mathrm{meV}$. (Both these are above the Raman energy of $165 \mathrm{meV}$.) Wight tentatively suggested that the defect was an acceptor-vacancy pair. No other author has studied this system.

The acceptor impurity, boron, has all its atomic spectral transitions in the ultraviolet. Hence $\mathrm{F}$ and $\mathrm{G}$ may derive from these transitions, in the free atom, particularly from the intense doublet at $4.96 \mathrm{eV}$. The local mode phonons suggest a defect with a mass lower than carbon, or increased force constants. A boron-vacancy pair seems unlikely, since vacancy-related defects usually have low phonon energies (e.g. GR1, $\mathrm{H} 3$, the $1.944 \mathrm{eV}$ centre). The centre could be a boron interstitial or a boron substitutional atom which has trapped a carbon interstitial.

Further work is clearly necessary on this interesting and neglected centre. In particular, it is not certain that $F$ and $G$ are due to the same defect. It may also have a bearing on whether the GR defect is a donor, and why the acceptor infrared absorption disappears in irradiated semiconducting diamonds ( $\$ \S 3.2 .3$ and 4.2.1). 
4.2.8. The tetragonal defect ( 736 and $667 \mathrm{~nm}, 1.685$ and $1.859 \mathrm{eV}$ ). These two zerophonon lines are observed in absorption in all types of diamond, but not apparently in cathodoluminescence (figure 19). They were incorrectly assumed to be part of the GR1 system by some authors, but they anneal out at about $700 \mathrm{~K}$ whereas GR1 does not and they do not appear in the GR1 excitation spectrum. The $1.685 \mathrm{eV}$ line freezes out below $80 \mathrm{~K}$, indicating that it is a transition from an energy level about $6 \mathrm{meV}$ above the ground state (Wight et al 1971, Clark and Walker 1972, 1973, Davies 1974a, 1977b, Walker 1977c).

A radiation-damage defect seen in EPR (the R2 system (Faulkner and Lomer 1962, Lomer and Wild 1973)) also anneals at about $700 \mathrm{~K}$ (Clark et al 1964) and Walker $(1977 \mathrm{c})$ has shown that it correlates in intensity with both the 1.685 and $1.859 \mathrm{eV}$ lines. Furthermore R2 has tetragonal symmetry, and uniaxial stress has indicated this symmetry for the optical lines (figure 27). Hence the optical and EPR centres must be

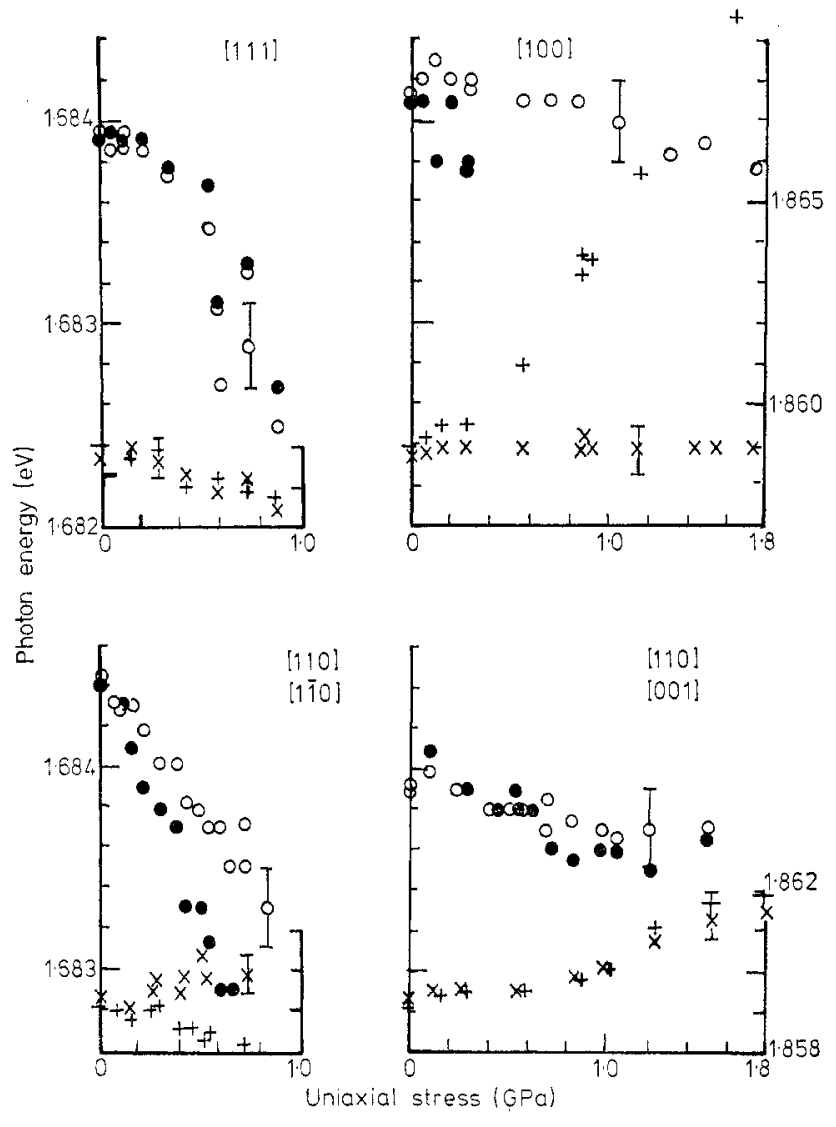

Figure 27. The 1.685 (circles) and $1.859 \mathrm{eV}$ (crosses) absorption lines under $\langle 100\rangle$, $\langle 111\rangle$ and $\langle 110\rangle$ stresses. Open circles and $x$ indicate parallel polarisation, and filled circles and + indicate perpendicular polarisation. Note the similarity in the splitting patterns, and in particular that parallel and perpendicular components are not resolved under $\langle 111\rangle$ stress and under [110] stress, [001] view-a result characteristic of an $A$ to $A$ transition in tetragonal symmetry. The $1.685 \mathrm{eV}$ perpendicular component under $\langle 100\rangle$ stress (filled circles) is too weak to observe above $0.4 \mathrm{GPa}$. Not that the left-hand scale applies to circles, the right-hand scale to crosses (Walker 1975). 
one and the same. The oscillator strengths for the optical lines can be estimated as $10^{-2}$, indicating allowed electric dipole transitions.

The identity of the defect has not been established; it is probably an interstitial complex involving an impurity (Walker 1977c). Further work is desirable, particularly in EPR.

\subsection{Irradiated and annealed diamond}

4.3.1. The $H$ lines. These absorption lines were investigated by Clark et al (1956b) in irradiated and annealed type Ia diamonds. The more intense ones (H2, H3 and H4) have been much studied and are due to three independent but related centres. H13 may be a transition at the $\mathrm{H} 3$ defect (Clark and Norris 1970). Nothing is known of the other $\mathrm{H}$ lines.

4.3.1.1. The $\mathrm{H} 2$ centre $(991.8 \mathrm{~nm}, 1 \cdot 25 \mathrm{eV})$. This centre is produced by heating irradiated diamonds containing more than $10^{25}$ nitrogen atoms $\mathrm{m}^{-3}$. The anneal temperature $(900 \mathrm{~K})$ seems to be critical; lower or higher temperatures do not result in $\mathrm{H} 2$.

Heating pre-annealed diamonds at temperatures of $500-600 \mathrm{~K}$ in the dark enhances $\mathrm{H} 2$ and reduces $\mathrm{H} 3$; illumination with mercury light $(2.84$ or $3.40 \mathrm{eV})$ reverses the process. Figure 22 shows this in a diamond containing GR1 and ND1 also, but it happens when only $\mathrm{H} 2$ and $\mathrm{H} 3$ are present. $\mathrm{H} 2$ cannot be produced in strength in type Ib or type II diamonds, and there is apparently no EPR associated with it (du Preez 1965, Dyer and du Preez 1967).

Wight (1968) has observed in cathodoluminescence a zero-phonon line at $1.264 \mathrm{eV}$ with a $60 \mathrm{meV}$ phonon replica in an irradiated and annealed diamond. It is possible that this is the emission counterpart of the $\mathrm{H} 2$ absorption system. No high-resolution H2 absorption spectrum has been published.

By analogy with Davies' (1977b) suggestion for ND1 and GR1, it may be that H3 and $\mathrm{H} 2$ are different charge states of the same defect, since they also show complementary bleaching behaviour. If so, at least one charge state will be paramagnetic, and it should be possible to detect hyperfine interactions due to the two nitrogen atoms contained in the $\mathrm{H} 3$ centre. The model suggested for $\mathrm{H} 2$ by Dyer and du Preez, a vacancy and an interstitial trapped at a 'nitrogen' platelet, now seems unlikely.

4.3.1.2. The $H 3$ centre $(503.2 \mathrm{~nm}, 2.464 \mathrm{eV})$. The $\mathrm{H} 3$ centre is one of the most studied in diamond (figure 28). Its properties have been reviewed recently by Davies (1977c). It can be observed in absorption, photoluminescence and cathodoluminescence after irradiation followed by annealing at $800 \mathrm{~K}$ or above, and is stable to at least $1500 \mathrm{~K}$. Although strongest in type Ia crystals, it has been observed in luminescence in types $\mathrm{Ib}$ and IIa, though not in type II b. The centre has generally been assumed to be a vacancy trapped at a nitrogen aggregate, since it appears when the GR1 centre anneals out, and this is still the current view (Clark et al 1956b, Dyer and Matthews 1957, Dean et al 1960, Wight 1968, Davies 1976).

The symmetry of the centre is either rhombic I or monoclinic I (table 4), most likely the former. The first (polarised luminescence) experiments indicated a $\pi\langle 110\rangle$ oscillator at a $\langle 110\rangle$ centre, provided that low excitation energy $(2.84 \mathrm{eV})$ was used (Elliott et al 1958, Clark et al 1962, Clark and Norris 1970). The most recent uniaxial stress results (Davies et al 1976) are in excellent agreement with rhombic I 


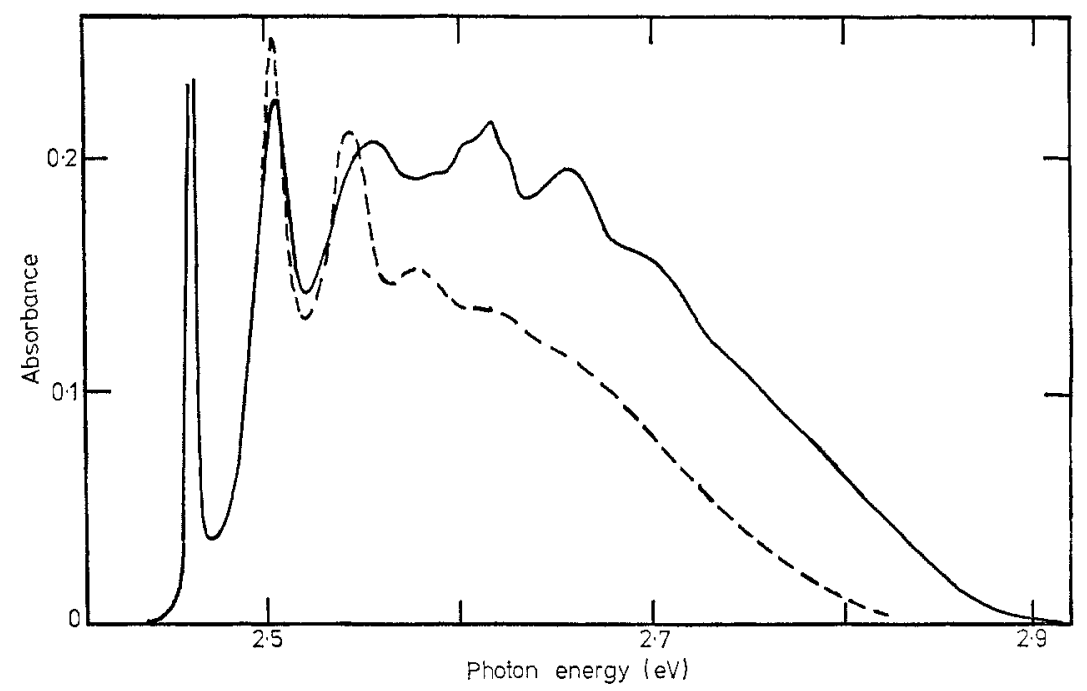

Figure 28. $80 \mathrm{~K}$ absorption spectrum of $\mathrm{H3}$ (full curve) compared with mirror image of luminescence (broken curve) (after Davies et al 1976).

symmetry. However, there is still a problem in that the Stark effect (Kaplyanskii et al 1971) indicates inversion symmetry, whereas $C_{2 \mathrm{v}}$, the only rhombic subgroup of $T_{d}$, is inversionless.

The uniaxial stress work of Davies et al revealed a forbidden transition at the $\mathrm{H} 3$ centre. It appears under $\langle 110\rangle$ and $\langle 111\rangle$ stress, and is a transition from the $A_{1}$ ground state to an $A_{1}$ excited state at $2.479 \mathrm{eV}, 16 \mathrm{meV}$ above the $B_{1}$ state at $2.463 \mathrm{eV}$. This transition is responsible for the departure from mirror symmetry of the absorption and luminescence bands (figure 28). In luminescence at $80 \mathrm{~K}$ the $2.479 \mathrm{eV}$ level is not populated; the emission spectrum is simpler and can be fitted by totally symmetric phonon coupling (Davies 1974b, Davies et al 1976). The phonon energies in absorption and emission are slightly different (figure 28), as they are at the GR1 centre (Nedzvetskii and Gaisin 1974b, Davies et al 1976). The current model for H3 is therefore a vacancy (i.e. the GR defect) trapped by a nitrogen pair (figure 6); to have rhombic I symmetry the vacancy must exchange with one of the nitrogens, giving an $\mathrm{N}-\mathrm{V}-\mathrm{N}$ arrangement (Davies 1976). The Wedlake model for $\mathrm{H3}$ (see Clark and Mitchell 1971), an N-V pair, was based on Runciman's (1965) incorrect uniaxial stress results, and is now untenable.

Many authors have observed weak $\mathrm{H} 3$ luminescence from nominally unirradiated diamond. Recent topographical studies (Hanley et al 1977) demonstrates that it is emitted preferentially from slip traces, dislocations and platelets. The luminescence appears to be intrinsic to these features rather than to $\mathrm{H} 3$ defects decorating them. Furthermore the $\mathrm{H} 3$ centres have been produced without any radiation damage ( $\mathrm{H} 3$ and $\mathrm{H} 4$ can occur in the radiation-damaged rinds of some diamonds, but only at depths of less than $30 \mu \mathrm{m}$, corresponding to the range of $\alpha$-particles). These facts carry implications for the structure of these defects. For example, both the Lang platelet model and the Davies $\mathrm{H} 3$ model involve pairs of nitrogen atoms (figure 6) (Walker 1977a). EPR centres observed particularly in plastically deformed diamonds have been shown to contain two nitrogen atoms (Loubser and Wright 1973b, Shcherbakova et al 1975, Welbourn and Woods 1977); a possible connection with H3 should be investigated. 
We have seen that $\mathrm{H} 13$ appears in the excitation spectrum of $\mathrm{H} 3$ (Clark and Norris 1970) and is therefore probably a transition at the same centre. H2 and H3 show complementary bleaching behaviour ( $\$ 4.3 .1 .1$ and figure 22). Hence they may be different charge states of the same defect. H4 is an independent centre, but apparently very similar to $\mathrm{H} 3$, as are other centres with zero-phonon lines at 2.417 and $2.536 \mathrm{eV}$ (Davies 1977c, de Sa and Davies 1977). The other $\mathrm{H}$ lines may or may not be related to $\mathrm{H} 2, \mathrm{H} 3$ and $\mathrm{H} 4$; correlation studies and excitation spectra are necessary. A luminescence line at $2 \cdot 3 \mathrm{eV}$ apparently accompanies $\mathrm{H} 3$ (Dean et al 1960, Wight 1968, Sobolev et al 1969b, Collins 1974) and could be a transition between excited states of the centre. Because $\mathrm{H} 3$ can be bleached, it must be paramagnetic in at least one charge state, though du Preez (1965) was unable to detect any EPR associated with either $\mathrm{H} 3$ or $\mathrm{H} 2$.

Two other zero-phonon lines, due to the $3 \mathrm{H}$ and $\mathrm{S} 1$ centres, occur at $503 \mathrm{~nm}$, and the $3 \mathrm{H}$ centre also has rhombic I symmetry. As we have already seen, the three centres are distinct but may be related; further work is necessary.

4.3.1.3. The $H 4(2.498 \mathrm{eV}), 2.417$ and $2.536 \mathrm{eV}$ bands. It is convenient to discuss these three centres together because of their similarity to each other and to $\mathrm{H} 3$ (figure 29). All of them are produced by irradiation and $900 \mathrm{~K}$ annealing of type IaB diamonds. $\mathrm{H} 4$ is stable to at least $1200 \mathrm{~K}$. Unlike $\mathrm{H} 3$, it has not been observed in synthetic crystals, nor in unirradiated diamond (Clark et al 1956b, Dyer and Matthews 1957, Nedzvetskii and Dymke 1970, Sobolev et al 1969b, de Sa and Davies 1977, Hanley et al 1977).

Davies (1972b) showed that $\mathrm{H} 4$ was produced when the B nitrogen aggregate
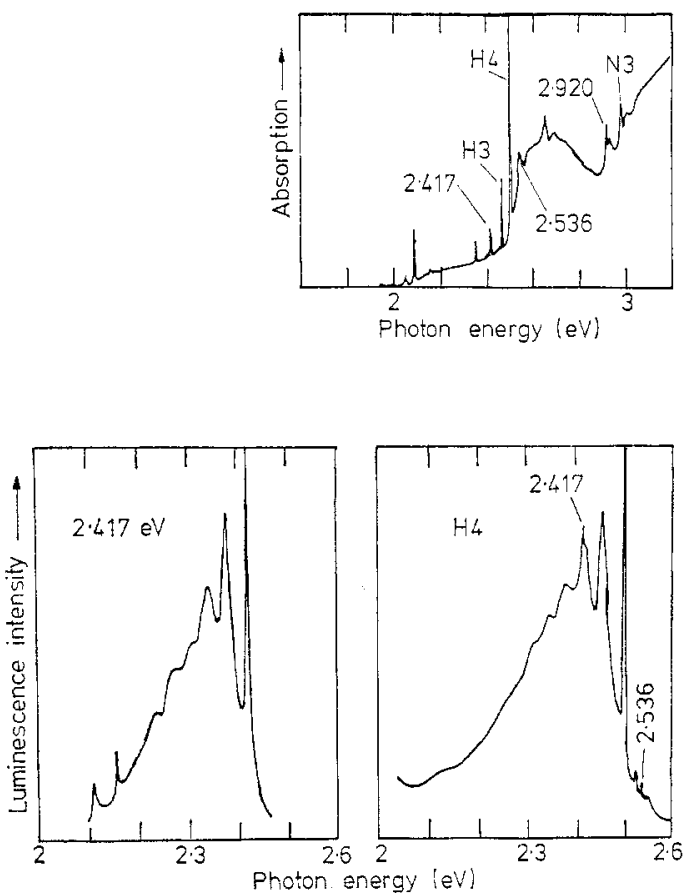

Figure 29. Absorption (upper) and photoluminescence (lower) spectra of the H4, 2.417 and $2.536 \mathrm{eV}$ defects. Various other independent zero-phonon lines can be seen (after de Sa and Davies 1977). 
trapped a radiation damage product (now thought to be the vacancy). Runciman (1965) was unable to identify its symmetry, but polarised luminescence (Clark and Norris 1971) indicated either rhombic I or monoclinic I.

de Sa and Davies (1977) have shown that $\mathrm{H} 4$ and the $2.417 \mathrm{eV}$ and $2.536 \mathrm{eV}$ centres all have monoclinic I symmetry (with stress coupling coefficients very similar to those of the $\mathrm{H} 3$ centre). Although they do not correlate quantitatively, and hence are distinct centres, they typically occur with relative intensities 100:10:2. H4 was investigated in most detail and was shown to have a pair of nearly degenerate excited states, to the upper of which transitions are forbidden. This results in a departure from mirror symmetry; a peak at $154 \mathrm{meV}$ in absorption does not appear in the luminescence spectrum (figure 29), exactly like $\mathrm{H} 3$ (figure 28 ). The $2 \cdot 417 \mathrm{eV}$ centre also shows this asymmetry.

The first phonon peak in absorption is at a higher energy shift than its counterpart in luminescence (de Sa and Davies 1977), a characteristic shared by H3 and the GR1 centre (Nedzvetskii and Gaisin 1974b). This, and the evidence already recounted, strongly suggests that all these centres are aggregates of nitrogen atoms which have trapped a vacancy (i.e. the GR defect) (de Sa and Davies 1977).

Further work could involve the Stark effect, to pin down the point group of the centres (suggested to be $\mathrm{C}_{1 \mathrm{~h}}$ rather than $\mathrm{C}_{2}$ or $\mathrm{C}_{2 \mathrm{~h}}$ by de $\mathrm{Sa}$ and Davies) and investigation of possible correlations with other nitrogen-dependent centres (e.g. N3, N9 and the EPR centres described by Shcherbakova et al (1975)).

4.3.2. The $575 \mathrm{~nm}$ system $(2.15 \mathrm{eV})$. This system (figure 30 ) was discovered by Ralph (1960) using cathodoluminescence. It is produced in all diamonds by irradiation followed by annealing, usually at $900-1100 \mathrm{~K}$, though lower temperatures may suffice, and anneals out at $1400 \mathrm{~K}$. The luminescence can also be photo-excited. In some crystals the cathodoluminescence is present before laboratory irradiation (Ralph 1960, Wight 1968, Davies 1970b, Collins 1977, Hanley et al 1977).

Alternatively, continued exposure to the cathodoluminescence electron beam after irradiation will induce and enhance the luminescence, even at $80 \mathrm{~K}$. This

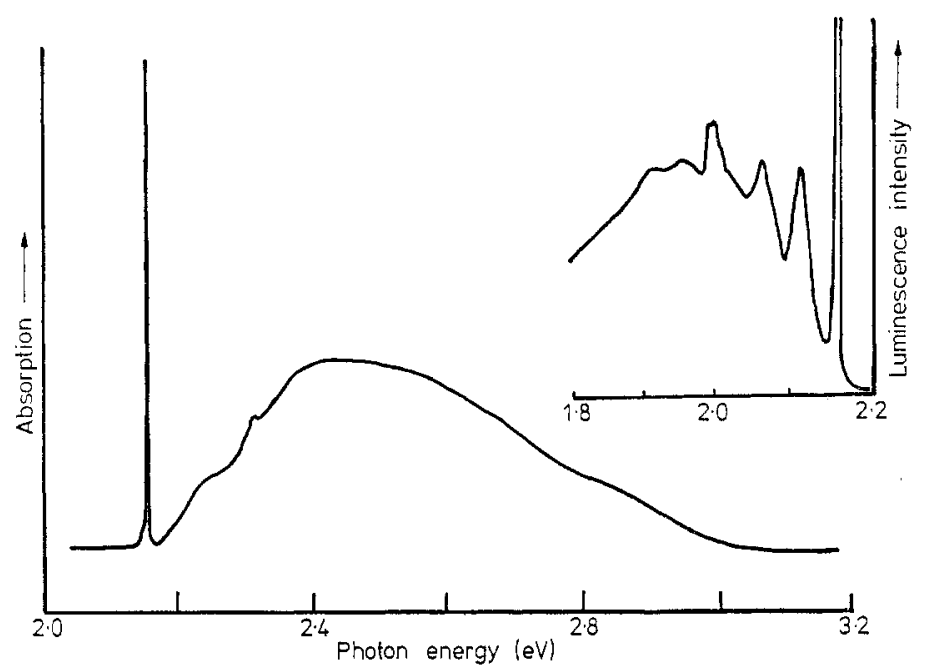

Figure 30. Absorption and cathodoluminescence spectra of the $575 \mathrm{~nm}$ system (after Wight 1968, Davies 1977d). 
suggests an athermal creation mechanism (Wight 1968, Davies 1970b, 1975b). On one occasion the $3 \mathrm{H}$ cathodoluminescence was observed to disappear, the $575 \mathrm{~nm}$ system replacing it (Hanley et al 1977). This suggests that the latter is created when $3 \mathrm{H}$ anneals, or that they are due to different charge states of the same defect; consistent with these possibilities both $3 \mathrm{H}$ and the $575 \mathrm{~nm}$ system can be created in all diamonds.

Davies (1972b) has observed a weak $575 \mathrm{~nm}$ zero-phonon line in absorption in types Ia and $\mathrm{Ib}$ crystals. Hence the transition cannot occur between two excited states. The symmetry of the centre is trigonal, the transition being between an A excited state and an E ground state. A low-lying A level is strongly coupled to the $\mathrm{E}$ state by stress, implying a Jahn-Teller effect; this is confirmed by the absorption band, which is not a mirror image of the luminescence (Davies 1977d).

No model is currently proposed for the centre. Dean's (1965) suggestion that it was the GR1 defect may now be discounted. Its symmetry, ubiquity and anneal temperature suggest a divacancy.

4.3.3. The $1.944 \mathrm{eV}$ system $(637.7 \mathrm{~nm})$. This centre (figure 31) was discovered by du Preez (1965). It is strongest in irradiated and annealed (900 K) type Ib diamonds, both natural and synthetic, though it also occurs in type Ia. The spectrum can be seen in absorption (crystals appear mauve in colour) and in photoluminescence (red), but not apparently in cathodoluminescence. Polarised luminescence indicated trigonal or (less likely) monoclinic I symmetry. Coupling is primarily to totally symmetric

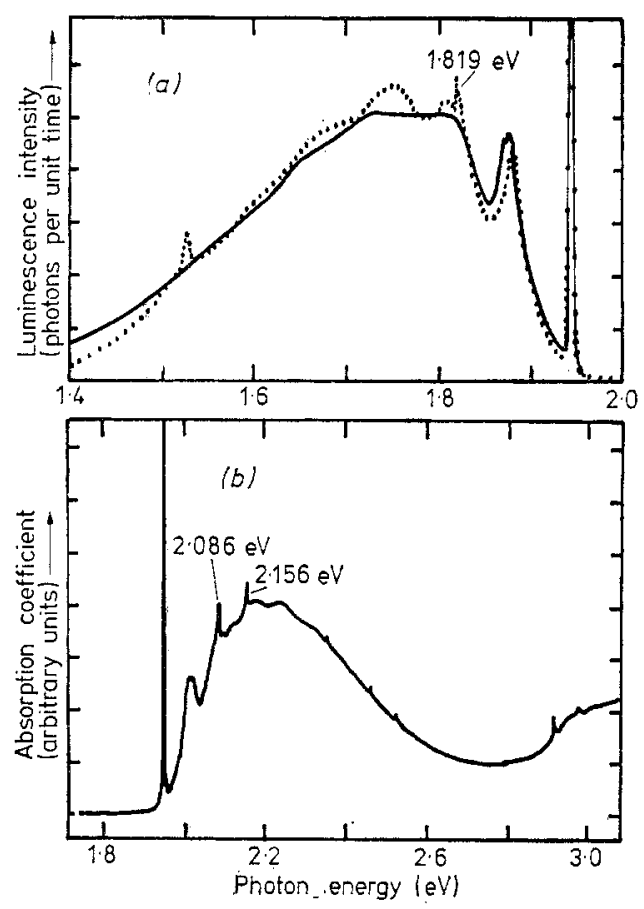

Figure 31. The $1.944 \mathrm{eV}$ centre: (a) measured $80 \mathrm{~K}$ luminescence spectrum (dots) compared with luminescence spectrum calculated from the absorption spectrum (full curve). (b) Measured $80 \mathrm{~K}$ absorption spectrum. Some independent zero-phonon lines are also present (after Davies and Hamer 1976). 
phonons; there is no evidence of Jahn-Teller effects (du Preez 1965, Clark and Norris 1971, Burton 1972, Collins 1974, Davies 1974b, 1977a).

du Preez noted that the $1.944 \mathrm{eV}$ centre appeared as GR1 and ND1 (thought to be vacancies) annealed out. His model, a vacancy trapped at a single nitrogen atom, has been confirmed by Davies and Hamer (1976), whose uniaxial stress experiments show that the $1.944 \mathrm{eV}$ line is due to an $\mathrm{A}$ (ground) to $\mathrm{E}$ transition at a trigonal defect. Although the $\mathrm{E}$ state is degenerate, uniaxial stress confirms that there is no JahnTeller effect. de Sa (1977) has shown that a zero-phonon line at $4.328 \mathrm{eV}$ is due to an A-E transition at a trigonal centre, and correlates with the $1.944 \mathrm{eV}$ line. It therefore must be a transition at the same centre.

Loubser and van Wyk (1977) have observed an EPR signal which appears to come from an excited triplet state of the $1.944 \mathrm{eV}$ centre; their work confirms the $\mathrm{N}-\mathrm{V}$ model.

Klingsporn et al (1970) identified a monoclinic $\left(\mathrm{C}_{2 \mathrm{~h}}\right)$ EPR centre containing nitrogen in type $\mathrm{Ib}$ crystals. These crystals fluoresced red and yellow under UV excitation (P E Klingsporn, personal communication). Shcherbakova et al (1972) have already associated the EPR centre with the yellow-fluorescing S1 system. It is possible that the $1.944 \mathrm{eV}$ system, since it involves nitrogen, is a different charge state of the $\mathrm{S} 1$ defect and gave rise to the red fluorescence in the crystals used by Klingsporn et al.

\section{Summary and conclusions}

I have included in this review the most important defects seen in optical absorption and luminescence in diamond. I have indicated where specific experiments are needed to clear up ignorance or disagreement, and where there are possible correspondences between optical and EPR centres. The last few years have seen a great deal of progress in identifying defects, but there is still much work to be done. Combined optical and EPR experiments are particularly promising. Doped synthetic crystals will permit additional advances, though the area of 'diamond electronics' is not in the foreseeable future.

The relevance of the work on diamond to the controversy about how to calculate the energy levels of 'deep' defects in semiconductors has already been mentioned. It is to be hoped that recent experimental verifications of 'defect molecule' predictions will stimulate further calculations.

\section{Acknowledgments}

I would like to thank the Royal Society for the award of a European Programme Fellowship with the Groupe de Physique des Solides de l'Ecole Normale Supérieure, Université Paris VII, during the tenure of which most of this review was written, and Jacques Bourgoin for making my stay so pleasant and profitable. I am indebted to Professor $\mathrm{H} \mathrm{H}$ Hopkins, FRS, for making facilities available at the J J Thomson Physical Laboratory, University of Reading, where the review was completed.

I am grateful to the colleagues who have given me help and advice, or have commented on the manuscript: in particular, C D Clark, A T Collins, G Davies, J E Lowther, E W J Mitchell, R C Newman and A M Stoneham. 
The authors and publishers who have generously given me permission to reproduce their diagrams here are acknowledged in the captions. I am grateful to Mme Girard for preparing the typescript and to my wife for preparing the diagrams.

\section{References}

Anastassakis E 1969 Phys. Rev. 186 760-7

Angress J F, Goodwin A R and Smith S D 1968 Proc. R. Soc. A 308 111-24

Astier M and Bourgoin J C 1976 Proc. 13th Int. Conf. on Physics of Semiconductors, Rome ed F G Fumi (Amsterdam: North-Holland) pp599-602

Bagguley D M S, Vella-Coleiro G, Smith S D and Summers C J 1966 F. Phys. Soc. Fapan Suppl. $21244-8$

Berman R (ed) 1965 The Physical Properties of Diamond (Oxford: Clarendon)

Berman R, Hudson P RW and Martinez M 1975 F. Phys. C: Solid St. Phys. 8 L430-4

Berman R and Martinez M 1976 Diamond Research 1976 (London: Industrial Diamond Information Bureau) pp 7-13

Bourgoin J C, Brosious PR, Kim YM, Corbett JW and Chrenko RM 1972 Phil. Mag. 26 $1167-78$

Bourgoin J C and Massarani B 1976 Phys. Rev. B 14 3690-4

Bourgoin J C and Walker J 1976 Industrial Diamond Review, October 1976 (London: Industrial Diamond Information Bureau) pp362-7

Bundy F P, Hall H T, Strong HM and Wentorf R H 1955 Nature 176 51-5

Bundy FP, Strong HM and Wentorf RH 1973 Chemistry and Physics of Carbon vol 10, ed PL Walker and P A Thrower (New York: Marcel Dekker) pp213-63

Burls J (ed) 1967 Science and Technology of Industrial Diamonds vol 1 and 2

Bursill L A, Barry J, Hudson P R W and Phakey P P 1977 Diamond Conf., Reading unpublished Burton B 1972 Diamond Conf., Bristol unpublished

Cannon P 1971 F. Phys. Chem. 75 2696-8

Caticha-Ellis S and Cochran W 1958 Acta Crystallogr. 11 245-9

Charette J J 1961a Physica 27 1061-73

— 1961b F. Chem. Phys. 35 1906-7

Chrenko RM 1973 Phys, Rev. B 7 4560-7

Chrenko RM, Strong HM and Tuft RE 1971 Phil. Mag. 23 313-8

Chrenko R M, Tuft RE and Strong H M 1977 Nature 270 141-4

Clark CD 1965 The Physical Properties of Diamond ed R Berman (Oxford: Clarendon) pp295-324

Clark CD, Ditchburn RW and Dyer H B 1956a Proc. R. Soc. A 234 363-81 1956b Proc. R. Soc. A $23775-89$

Clark CD, Duncan I, Lomer J N and Whippey P W 1964 Proc. Br. Ceram. Soc. 1 85-92

Clark C D, Kemmey P J and Mitchell EW J 1961 Disc. Faraday Soc. 31 96-106

Clark C D, Kemmey P J, Mitchell EW J and Henvis B W 1960 Phil. Mag. 5 127-39

Clark CD, Maycraft GW and Mitchell EW J 1962 \%. Appl. Phys. Suppl. 33 378-82

Clark CD and Mitchell EW J 1971a Radiat. Effects 9 219-34

$1971 \mathrm{~b}$ Proc. Int. Conf. on Radiation Effects in Semiconductors, Albany, NY, 1970 ed J W Corbett and GD Watkins (New York: Gordon and Breach) pp257-72

1976 Radiation Effects in Semiconductors. Inst. Phys. Conf. Ser. No. 31 (Bristol: The Institute of Physics) pp45-57

Clark C D, Mitchell E W J and Parsons B J 1979 The Properties of Diamond ed J E Field (New York: Academic) pp23-77

Clark CD and Norris CA 1970 F. Phys. C: Solid St. Phys. 3 651-8

— 1971 F. Phys. C: Solid St. Phys. 4 2223-9

Clark CD and Walker J 1972 Diamond Research 1972 (London: Industrial Diamond Information Bureau) pp2-5

1973 Proc. $R$. Soc. A 334 241-57

Collins A T 1974 Industrial Diamond Review, April 1974 (London: Industrial Diamond Information Bureau) pp131-7 
1977 Radiation Effects in Semiconductors 1976. Inst. Phys. Conf. Ser. No 31 (Bristol: The Institute of Physics) pp346-53

1978 Diamond Research 1978 (London: Industrial Diamond Information Bureau) pp37-45

Collins A T, Dean P J, Lightowlers E C and Sherman W F 1965 Phys. Rev. 140 A1272-4

Collins A T and Lightowlers E C 1968 Phys. Rev. 171 843-55

1979 The Properties of Diamond ed J E Field (New York: Academic) pp79-105

Collins A T, Lightowlers E C and Dean P J 1969 Phys. Rev. 183 725-30

Collins RJ and Fan HY 1954 Phys. Rev. 93 674-8

Coulson C A and Larkins F P 1971 f. Phys. Chem. Solids 32 2245-57

Crossfield M 1974 Diamond Conference, Oxford unpublished

Crowther PA and Dean P J 1967 \%. Phys. Chem. Solids 28 1115-36

Crowther PA, Dean PJ and Sherman W F 1967 Phys. Rev. 154 772-85

Curie D 1975 Optical Properties of Ions in Solids ed B di Bartolo (New York: Plenum) pp71-105

Davies G 1970a Nature 228758

—1970b Diamond Conf., Oxford unpublished

1971 Phonons. Int. Conf. Rennes, France ed M A Nusimovici (Paris: Flammarion) pp382-6

— 1972a Diamond Research 1972 (London: Industrial Diamond Information Bureau) pp21-30

-1972b \%. Phys. C: Solid St. Phys. 5 2534-42

1974a Proc. R. Soc. A 336 509-23

- 1974b \%. Phys. C: Solid St. Phys. 7 3797-809

— 1975a F. Phys. C: Solid St. Phys. 8 2448-54

1975b Diamond Research 1975 (London: Industrial Diamond Information Bureau) pp13-7

1975c Diamond Conf., Cambridge unpublished.

1976 F. Phys. C: Solid St. Phys. 9 L537-42

1977a Chemistry and Physics of Carbon vol 13, ed P W Philips and P A Turner (New York:

Marcel Dekker) pp1-143

- $1977 \mathrm{~b}$ Nature $269498-500$

- 1977c Diamond Research 1977 (London: Industrial Diamond Information Bureau) pp15-24

1977d Diamond Conf., Reading unpublished

Davies G and Foy CP 1978 F. Phys. C: Solid St. Phys. 11 L547-53

Davies G and Hamer MF 1976 Proc. R. Soc. A 348 285-98

Davies G and Lightowlers E C 1970 F. Phys. C: Solid St. Phys. 3 638-50

Davies G, Nazaré MH and Hamer MF 1976 Proc. R. Soc. A 351 245-65

Davies G and Penchina CM 1974 Proc. R. Soc. A 338 359-74

Davies G and Summersgill I 1973 Diamond Research 1973 (London: Industrial Diamond Information Bureau) pp6-15

Dawber PG and Elliott RJ 1963 Proc. Phys. Soc. 81 453-60

Dean P J 1965 Phys. Rev. 139 A588-602

Dean P J, Kennedy P J and Ralph JE 1960 Proc. Phys. Soc. 76 670-87

Dean PJ and Male J C 1964a Proc. R. Soc. A 277 330-47

- 1964b F. Phys. Chem. Solids 25 1369-83

Denham P, Lightowlers E C and Dean PJ 1967 Phys. Rev. 161 762-8

de Sa E S 1977 Diamond Conf., Reading unpublished.

de Sa ES and Davies G 1977 Proc. R. Soc. A 357 231-51

Douglas I N and Runciman WA 1977a F. Phys. C: Solid St. Phys. 10 2253-9

— 1977b Phys. Chem. Minerals 1 129-36

Dunn D 1976 Diamond Conf., Bristol unpublished

du Preez L 1965 PhD Thesis University of the Witwatersrand, Johannesburg

Dyer H B and du Preez L 1965 F. Chem. Phys. 42 1898-906

1967 Science and Technology of Industrial Diamonds vol 1, ed J Burls (London: Industrial

Diamond Information Bureau) pp23-6

Dyer H B and Ferdinando P 1966 Br. F. Appl. Phys. 17 419-20

Dyer H B and Matthews I G 1957 Proc. R. Soc. A 243 320-35

Dyer H B, Raal F A, du Preez L and Loubser J HN 1965 Phil. Mag. 11 763-74

Elliott RJ 1960 Proc. Phys. Soc. 76 787-91

Elliott RJ, Matthews R G and Mitchell EW J 1958 Phil. Mag. 3 360-9

Englman R 1971 The Yahn-Teller Effect in Molecules and Crystals (London: Wiley Interscience) 
Evans T 1973 Diamond Research 1973 (London: Industrial Diamond Information Bureau) pp2-5

1976 Contemp. Phys. 17 45-70

Evans T and Phaal C 1962a Proc. R. Soc. A 270 538-52

- 1962b Phil. Mag. 7 843-6

Evans T and Rainey P 1975a Diamond Research 1975 (London: Industrial Diamond Information Bureau) pp29-34 1975b Proc. $R$. Soc. A 344 111-30

Evans T, Stephenson R, Martin G D and Brozel M R 1976 Diamond Conf., Bristol unpublished

Evans T and Wild R K 1967 Phil. Mag. 15 447-51

Farrer R G 1974 PhD Thesis University of the Witwatersrand, Johannesburg

Farrer RG and Vermeulen LA 1972 F. Phys. C: Solid St. Phys. 5 2762-8

Faulkner EA and Lomer J N 1962 Phil. Mag. 7 1995-2002

Fesq HW, Bibby DM, Erasmus CS, Kable EJD and Sellschop JPF 1975 Physics and Chemistry of the Earth vol 9, ed LH Ahrens, J B Dawson, AR Duncan and AJ Erlank pp817-36

Fesq HW, Bibby DM, Sellschop J PF and Watterson JIW 1973 F. Radioanalyt. Chem. 17 195-216

Field JE (ed) 1979 The Properties of Diamond (New York: Academic)

Frank F C 1956 Proc. $R$. Soc. A 237 168-74

- 1964 Proc. Phys. Soc. 84 745-8

Grenville-Wells H J 1952 Proc. Phys. Soc. B 65 313-20

Ham F S 1972 Electron Paramagnetic Resonance ed S Geschwind (New York: Plenum) pp1-119

Hanley P L, Kiflawi I and Lang A R 1977 Phil. Trans. R. Soc. A 284 329-68

Hardy J R 1962a Proc. Phys. Soc. 79 1154-60

- 1962b Phil. Mag. 7 953-60

Hardy J R, Smith S D and Taylor W 1962 Proc. Int. Conf. on Physics of Semiconductors, Exeter (London: Institute of Physics) pp521-8

Heine V 1964 Group Theory in Quantum Mechanics (Oxford: Pergamon)

Henry NFM and Lonsdale K (ed) 1965 International Tables for X-ray Crystallography vol 1 (Birmingham: Kynoch Press) p340

Hoerni J A and Wooster W A 1955 Acta Crystallogr. 8 187-94

Hughes AE and Runciman W A 1967 Proc. Phys. Soc. 90 827-38

Il'in VE, Sobolev EV and Yur'eva O P 1971 Sov. Phys.-Solid St. 12 2159-61

Kaiser W and Bond W L 1959 Phys. Rev. 115 857-63

Kaplyanskii A A 1964a Opt. Spectrosc. 16 329-37

- 1964b Opt. Spectrosc. 16 557-65 1967 7. Physique 28 Suppl. C4 39-48

Kaplyanskii AA, Kolyshkin V I and Medvedev V N 1970 Sov. Phys.-Solid St. 12 1193-5

Kaplyanskii AA, Kolyshkin VI, Medvedev V N and Skvortsov AP 1971 Sov. Phys.-Solid St. 12 2867-72

Kemmey PJ and Wedepohl P T 1965 Physical Properties of Diamond ed R Berman (Oxford: Clarendon) pp325-55

Kiflawi I and Lang A R 1974 Phil. Mag. 30 219-23

— 1976 Phil. Mag. 33 697-701

- 1977 Nature $26736-7$

Klingsporn PE, Bell MD and Leivo W J 1970 f. Appl. Phys. 41 2977-80

Klyuev Yu A, Dudenkov Yu A and Nepsha V I 1973 Geochem. Int. 7 781-7

Klyuev Yu A, Naletov A M, Nepsha V I, Apishina N I and Bulygina T I 1977 Sov. Phys.-Solid St. 19 7-10

Klyuev Yu A, Nepsha VI and Bezrukov G N 1975a Sov. Phys. Semicond. 8 1053-4

Klyuev Yu A, Nepsha VI, Dudenkov Yu A, Zvonkov SD and Zubkov VM 1972 Sov. Phys. Dokl. 17 308-11

Klyuev Yu A, Nepsha VI and Naletov A M 1975b Sov. Phys.-Solid St. 16 2118-21

Klyuev Yu A, Rykov A N, Dudenkov Yu A and Zubkov V M 1970 Sov. Phys. Dokl. 14 1133-5

Lang A R 1964 Proc. Phys. Soc. 84 871-6 1977 Phil. Mag. 36 495-500

Lannoo M and Stoneham A M 1968 F. Phys. Chem. Solids 29 1987-2000

Larkins F P and Stoneham A M 1971 F. Phys. C: Solid St. Phys. 4 143-63 
Lax M and Burstein E 1955 Phys. Rev. 97 39-52

Lightowlers E C and Collins A T 1966 Phys. Rev. 151 685-8

1976 F. Phys. D: Appl. Phys. 9 951-63

Lightowlers E C, Collins A T, Denham P and Walsh P S 1968 Diamond Research 1968 (London: Industrial Diamond Information Bureau) pp11-24

Lightowlers EC and Dean PJ 1964 Diamond Research 1964 (London: Industrial Diamond Information Bureau) pp21-5

Lisoivan V I and Sobolev EV 1974 Sov. Phys. Dokl. 19 56-8

Lomer J N and Welbourn C M 1976 Radiation Effects in Semiconductors. Inst. Phys. Conf. Ser. No 31 (Bristol: The Institute of Physics) pp339-45

Lomer J N and Wild AMA 1971 Phil. Mag. 24 273-8

— 1973 Radiat. Effects $1737-44$

Lonsdale K and Smith H 1942 Proc. R. Soc. A 179 8-50

Loubser J H N 1977 Diamond Conf., Reading unpublished

Loubser J H N and Van Ryneveld W P 1967 Br. F. Appl. Phys. 18 1029-31

Loubser J H N and van Wyk J A 1977 Diamond Research 1977 (London: Industrial Diamond Information Bureau) pp11-4 1978 Rep. Prog. Phys. 41 1201-48

Loubser J H N and Wright A C J 1973a Diamond Research 1973 (London: Industrial Diamond Information Bureau) pp16-20

1973b F. Phys. D: Appl. Phys. 6 1129-41

Lowther JE 1975 F. Phys. C: Solid St. Phys. 8 3448-56

1976 Solid St. Commun. 20 933-5

- 1977 Phil. Mag. 36 483-93

Lowther JE and Stoneham A M 1978 \%. Phys. C: Solid St. Phys. 11 2165-9

Massarani B and Bourgoin J C 1976 Phys. Rev. B 14 3682-9

Melton CE and Giardini A A 1974 Am. Miner. 59 775-82 1976 Nature 263 309-10

Mitchell EWJ 1964 Diamond Research 1964 (London: Industrial Diamond Information Bureau) pp13-6

Nahum J and Halperin A 1962 F. Phys. Chem. Solids 23 345-58

Nazaré M H and Davies G 1977 Diamond Conf., Reading unpublished

Nedzvetskii DS and Dymke 1970 Opt. Spectrosc. 28 41-5

Nedzvetskii DS and Gaisin V A 1973a Sov. Phys.-Solid St. 15 427-8

1973b Sov. Phys.-Solid St. 14 2535-8

- 1974a Opt. Spectrosc. $36123-5$

—_ 1974b Sov. Phys.-Solid St. 16 145-6

1975 Sov. Phys.-Solid St. 16 1600-1

Nikitin AV, Somoilovich M I, Bezrukov G N and Vorozheikin KF 1969 Sov. Phys. Dokl. 13 $842-4$

Palmer DW 1961 PhD Thesis Reading University

Raal F A 1959 Proc. Phys. Soc. 74 647-9

Ralph J E 1960 Proc. Phys. Soc. 76 688-96

Raman C V and Nilakantan P 1940 Proc. Ind. Acad. Sci. A 11389

Ritter J T 1970 Solid St. Commun. 8 773-5

Robertson R, Fox J J and Martin A E 1934 Phil. Trans. R. Soc. A 232 463-535

1936 Proc. $R$. Soc. A 157 579-93

Runciman WA 1965 Proc. Phys. Soc. 86 629-36

1969 Physics of Solids in Intense Magnetic Fields ed ED Haidemenakis (New York: Plenum) pp344-58

Samoilovich M I, Bezrukov GN, Butuzov V P and Podol'skikh L D 1975 Sov. Phys. Dokl. 19 409-10

Sellschop JPF 1975 Diamond Research 1975 (London: Industrial Diamond Information Bureau) pp35-41

Sellschop J P F, Annegarn H J, Madiba C, Keddy RJ and Renan M J 1977 Diamond Research 1977 (London: Industrial Diamond Information Bureau) pp2-4

Sellschop J P F, Bibby D M, Erasmus CS and Mingay DW 1974 Diamond Research 1974 (London: Industrial Diamond Information Bureau) pp43-50

Shcherbakova M Ya, Sobolev EV and Nadolinnyi VA 1972 Sov. Phys. Dokl. 17 513-6 
Shcherbakova M Ya, Sobolev EV, Nadolinnyi VA and Aksenov VK 1975 Sov. Phys. Dokl $20725-8$

Shcherbakova, M Ya, Sobolev EV, Samsonenko ND and Aksenov V K 1969 Sov. Phys.-Solid St. 11 1104-6

Shul'man LA, Zaritskii I M and Podzyarei GA 1967 Sov. Phys.-Solid St. 8 1842-5

Smith SD 1961 Disc. Faraday Soc. 31 255-7

Smith S D and Hardy J R 1960 Phil. Mag. 5 1311-4

Smith S D, Hardy J R and Mitchell EW J 1962 Proc. Int. Conf. on Physics of Semiconductors, Exeter (London: Institute of Physics) pp529-34

Smith SD and Taylor W 1962 Proc. Phys. Soc. 79 1142-53

Smith W V, Sorokin P P, Gelles I L and Lasher G J 1959 Phys. Rev. 115 1546-52

Sobolev EV and Dubov Yu I 1975 Sov. Phys.-Solid St. 17 1142-4

Sobolev EV and Eliseev A P 1976 F. Struct. Chem. 17 802-4

Sobolev E V, Il'in V E, Gil'bert E I and Lenskaya S V 1969a Sov. Phys.-Solid St. 11 200-2

Sobolev E V, Il'in VE, Gil'bert E I, Lenskaya S V, Pronin VA, Pelekis LL, Mednis I V and Movalev VM 1970 F. Struct. Chem. 11 977-80

Sobolev EV, Il'in VE and Yur'eva OP 1969b Sov. Phys.-Solid St. $11938-43$

Sobolev EV and Lisoivan VI 1972 Sov. Phys. Dokl. 17 425-7

Sobolev EV, Lisoivan V I and Lenskaya SV 1968 Sov. Phys. Dokl. 12 665-8

Sobolev EV, Litvin Yu A, Samsonenko ND, Il'in VE, Lenskaya S V and Butuzov VP 1969c Sov. Phys.-Solid St. 10 1789-90

Solin S A and Ramdas A K 1970 Phys. Rev. B 1 1687-98

Stoneham AM 1975 Theory of Defects in Solids (Oxford: Clarendon)

— 1977a Solid St. Commun. 21 339-41

- 1977b Diamond Conf., Reading unpublished

Sturge MD 1967 Solid St. Phys. 20 91-211

Summersgill 1972 Diamond Conf., Bristol unpublished

Sutherland G B B M, Blackwell DE and Simeral W G 1954 Nature 174 901-4

Szigeti B 1963 F. Phys. Chem. Solids 24 225-37

Takagi M and Lang A R 1964 Proc. R. Soc. A 281 310-22

Tinkham M 1964 Group Theory and Quantum Mechanics (New York: McGraw-Hill)

Turk L A and Klemens P G 1974 Phys. Rev. B 9 4422-8

Vavilov V S 1975 Phys. Stat. Solidi a 31 11-26

Vavilov V S, Gukasyan M A, Guseva M I, Karatygina TA and Konorova E A 1974 Sov. Phys. Semicond. 8 471-3

Vavilov V S, Gukasyan MA, Guseva MI and Konorova EA 1972 Sov. Phys. Semicond. 6 741-6

Vavilov V S and Konorova EA 1976 Sov. Phys. Usp. 19 301-16

Vermeulen LA 1976 Diamond Research 1976 (London: Industrial Diamond Information Bureau) pp22-5

Vermeulen LA, Clark CD and Walker J 1974 Lattice Defects in Semiconductors. Inst. Phys. Conf. Ser. No 23 (Bristol: The Institute of Physics) pp294-300

Vermeulen LA and Farrer R G 1975 Diamond Research 1975 (London: Industrial Diamond Information Bureau) pp18-23

Walker J 1974 Lattice Defects in Semiconductors. Inst. Phys. Conf. Ser. No 23 (Bristol: The Institute of Physics) pp317-24

—1975 Diamond Conf., Cambridge unpublished

- 1976a Radiation Effects in Semiconductors. Inst. Phys. Conf. Ser. No 31 (Bristol: The Institute of Physics) pp510-1

- 1976b Nature 263275

- 1977a Nature $26713-4$

—_ 1977b F. Phys. C: Solid St. Phys. $103031-7$

- 1977c F. Phys. C: Solid St. Phys. $103867-76$

Walker J, Vermeulen LA and Clark CD 1974 Proc. R. Soc. A 341 253-66

Watkins GD 1965 Radiation Damage in Semiconductors (Paris: Dunod) pp97-113

Wedepohl P T 1957a Proc. Phys. Soc. B 70 177-85

- 1957b PhD Thesis Reading University

Weigel C, Peak D, Corbett J W, Watkins GD and Messmer R P 1973 Phys. Rev. B 8 2906-15

Welbourn CM and Davies G 1977 Diamond Conf., Reading unpublished 
Welbourn CM and Woods GS 1977 Diamond Conf., Reading unpublished Wight D R 1968 PhD Thesis King's College, University of London

Wight D R and Dean P J 1967 Phys. Rev. 154 689-96

Wight D R, Dean P J, Lightowlers E C and Mobsby CD 1971 F. Luminescence 4 169-93

Williams A W S, Lightowlers E C and Collins A T 1970 F. Phys. C: Solid St. Phys. 3 1727-35

Woods G S 1976 Phil. Mag. 34 993-1012

Woods G S and Lang A R 1975 F. Cryst. Growth 28 215-26 